\title{
Divergence of Carbonyl Ylide Reactions as a Function of Diazocarbonyl Compound and Aldehyde Substituent - Dioxolanes, Dioxolenes, and Epoxides
}

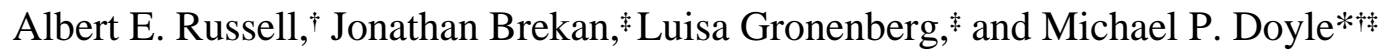 \\ $\dagger$ Department of Chemistry and Biochemistry, University of Maryland, College Park, \\ Maryland, 20742 \\ \$ Department of Chemistry, University of Arizona, Tuscon, Arizona 85721
}

\section{Supporting Information}

\section{Contents}

General Information S3

${ }^{1} \mathrm{H}$ NMR spectrum of compound 11a $\quad$ S4

${ }^{13} \mathrm{C}$ NMR spectrum of compound 11a S5

${ }^{1} \mathrm{H}$ NMR spectrum of compound 11b S6

${ }^{13} \mathrm{C}$ NMR spectrum of compound 11b S7

${ }^{1} \mathrm{H}$ NMR spectrum of compounds $\mathbf{1 1 b}+$ cis-10b S8

${ }^{13} \mathrm{C}$ NMR spectrum of compounds $\mathbf{1 1 b}+\boldsymbol{c i s}-\mathbf{1 0 b} \quad$ S9

${ }^{1} \mathrm{H}$ NMR spectrum of compound trans-10b $\quad \mathrm{S} 10$

${ }^{13} \mathrm{C}$ NMR spectrum of compound trans-10b S11

${ }^{1} \mathrm{H}$ NMR spectrum of compounds 11c + cis-, trans-10c S12

${ }^{13} \mathrm{C}$ NMR spectrum of compounds 11c + cis-, trans-10c S13

${ }^{1} \mathrm{H}$ NMR spectrum of compound 11d $\quad \mathrm{S} 14$

${ }^{13} \mathrm{C}$ NMR spectrum of compound 11d $\quad \mathrm{S} 15$

${ }^{1}$ H NMR spectrum of compound trans-10d $\quad$ S16

${ }^{13} \mathrm{C}$ NMR spectrum of compound trans-10d $\quad \mathrm{S} 17$

${ }^{1} \mathrm{H}$ NMR spectrum of compound cis-10d $\quad \mathrm{S} 18$

${ }^{13}$ C NMR spectrum of compound cis-10d $\quad \mathrm{S} 19$

${ }^{1} \mathrm{H}$ NMR spectrum of compound 11e + cis-10e $\quad$ S20 
${ }^{13} \mathrm{C}$ NMR spectrum of compound 11e + cis-10e S21

${ }^{1} \mathrm{H}$ NMR spectrum of compound trans-10e $\quad$ S22

${ }^{13} \mathrm{C}$ NMR spectrum of compound trans-10e S23

$\begin{array}{ll}{ }^{1} \mathrm{H} \text { NMR spectrum of compound cis-10e } & \text { S24 }\end{array}$

${ }^{13} \mathrm{C}$ NMR spectrum of compound cis-10e $\quad \mathrm{S} 25$

nOe of compound cis-10e at $\mathbf{6 . 1 4} \mathrm{ppm} \quad S 26$

$\begin{array}{lr}\text { nOe of compound cis-10e at } \mathbf{5 . 9 6} \text { ppm } & \text { S27 }\end{array}$

$\begin{array}{ll}{ }^{1} \mathrm{H} \text { NMR spectrum of compound cis-10f } & \text { S28 }\end{array}$

${ }^{13} \mathrm{C}$ NMR spectrum of compound cis-10f $\quad$ S29

$\begin{array}{ll}{ }^{1} \mathrm{H} \text { NMR spectrum of compound trans-10f } & \text { S30 }\end{array}$

${ }^{13} \mathrm{C}$ NMR spectrum of compound trans-10f $\quad$ S31

${ }^{1} \mathrm{H}$ NMR spectrum of compound 12a $\quad$ S32

${ }^{13} \mathrm{C}$ NMR spectrum of compound 12a $\quad$ S33

$\begin{array}{ll}{ }^{1} \mathrm{H} \text { NMR spectrum of compound 12b } & \mathrm{S} 34\end{array}$

${ }^{13} \mathrm{C}$ NMR spectrum of compound 12b $\quad$ S35

${ }^{1} \mathrm{H}$ NMR spectrum of compound 12c S36

${ }^{13} \mathrm{C}$ NMR spectrum of compound 12c S37

${ }^{1} \mathrm{H}$ NMR spectrum of compound 12d $\quad$ S38

${ }^{13} \mathrm{C}$ NMR spectrum of compound 12d $\quad$ S39

$\begin{array}{ll}{ }^{1} \mathrm{H} \text { NMR spectrum of compound 12e } & \text { S40 }\end{array}$

${ }^{13} \mathrm{C}$ NMR spectrum of compound 12e $\quad$ S41

${ }^{1} \mathrm{H}$ NMR spectrum of compound 13a $\quad$ S42

${ }^{13} \mathrm{C}$ NMR spectrum of compound 13a $\quad$ S43

$\begin{array}{ll}\text { nOe of compound 13a at } \mathbf{4 . 5 8} \mathrm{ppm} & \text { S44 }\end{array}$

nOe of compound 13a at $4.47 \mathrm{ppm} \quad \mathrm{S} 45$

$\begin{array}{ll}{ }^{1} \mathrm{H} \text { NMR spectrum of compound 13b } & \text { S46 }\end{array}$

${ }^{13} \mathrm{C}$ NMR spectrum of compound 13b $\quad$ S47

$\begin{array}{ll}{ }^{1} \mathrm{H} \text { NMR spectrum of compound 13c } & \text { S48 }\end{array}$

${ }^{13} \mathrm{C}$ NMR spectrum of compound 13c $\quad$ S49

$\begin{array}{ll}{ }^{1} \mathrm{H} \text { NMR spectrum of compound 13d } & \text { S50 }\end{array}$

${ }^{13} \mathrm{C}$ NMR spectrum of compound 13d $\quad$ S51 
${ }^{1} \mathrm{H}$ NMR spectrum of compound 13e $\quad$ S52

${ }^{13} \mathrm{C}$ NMR spectrum of compound 13e S53

${ }^{1} \mathrm{H}$ NMR spectrum of compound 13f $\quad$ S54

${ }^{13} \mathrm{C}$ NMR spectrum of compound $\mathbf{1 3 f} \quad$ S55

${ }^{1} \mathrm{H}$ NMR spectrum of compound 13g S56

${ }^{13} \mathrm{C}$ NMR spectrum of compound 13g $\quad$ S57

${ }^{1} \mathrm{H}$ NMR spectrum of compound 13h $\quad$ S58

${ }^{13} \mathrm{C}$ NMR spectrum of compound $\mathbf{1 3 h} \quad$ S59

${ }^{1}$ H NMR spectrum of compound 14a $\quad$ S60

${ }^{13} \mathrm{C}$ NMR spectrum of compound 14a $\quad$ S61

Table 1a. $\quad$ S62

General. ${ }^{1} \mathrm{H}$ NMR (250 MHz, $400 \mathrm{MHz}, 500 \mathrm{MHz}$ or $600 \mathrm{MHz}$ ) and ${ }^{13} \mathrm{C}$ NMR (62.5 $\mathrm{MHz}, 100 \mathrm{MHz}, 125 \mathrm{MHz}$ or $150 \mathrm{MHz}$ ) spectra were obtained from solutions of $\mathrm{CDCl}_{3}$. Chemical shifts are reported in ppm from tetramethylsilane (TMS). HRMS were obtained by HR-FAB method. Anhydrous THF was distilled from sodium/ benzophenone ketyl. Dichloromethane was passed through a solvent column prior to use and was not distilled. The solvent columns are composed of activated alumina (A-2). ${ }^{1}$ Dimethyl diazomalonate ${ }^{2}$ and methyl diazoacetoacetate ${ }^{3}$ were prepared according to previously reported procedures. Methyl 3-tert-Butyldimethylsilanyloxy-2-diazobut-3enoate was prepared according to a previously reported procedure. ${ }^{4}$

\section{References}

(1) Pangborn, A.B.; Giardello, M.A.; Grubbs, R.H.; Rosen, P.K.; Timmers, F.J. Organometallics 1996, 15, 1518.

(2) Tullis, J.S.; Helquist, P. Org. Syn. 1997, 74, 229.

(3) Davies, H.M.L.; Cantrell, W.R., Jr.; Romines, K.R.; Baum, J.S. Org. Syn. 1991, $70,93$.

(4) Davies, H.M.; Ahmed, G.; Churchill, M. J. Am. Chem. Soc. 1996, 118, 10774 


\section{M.P. Doyle et al. - Supporting Information}

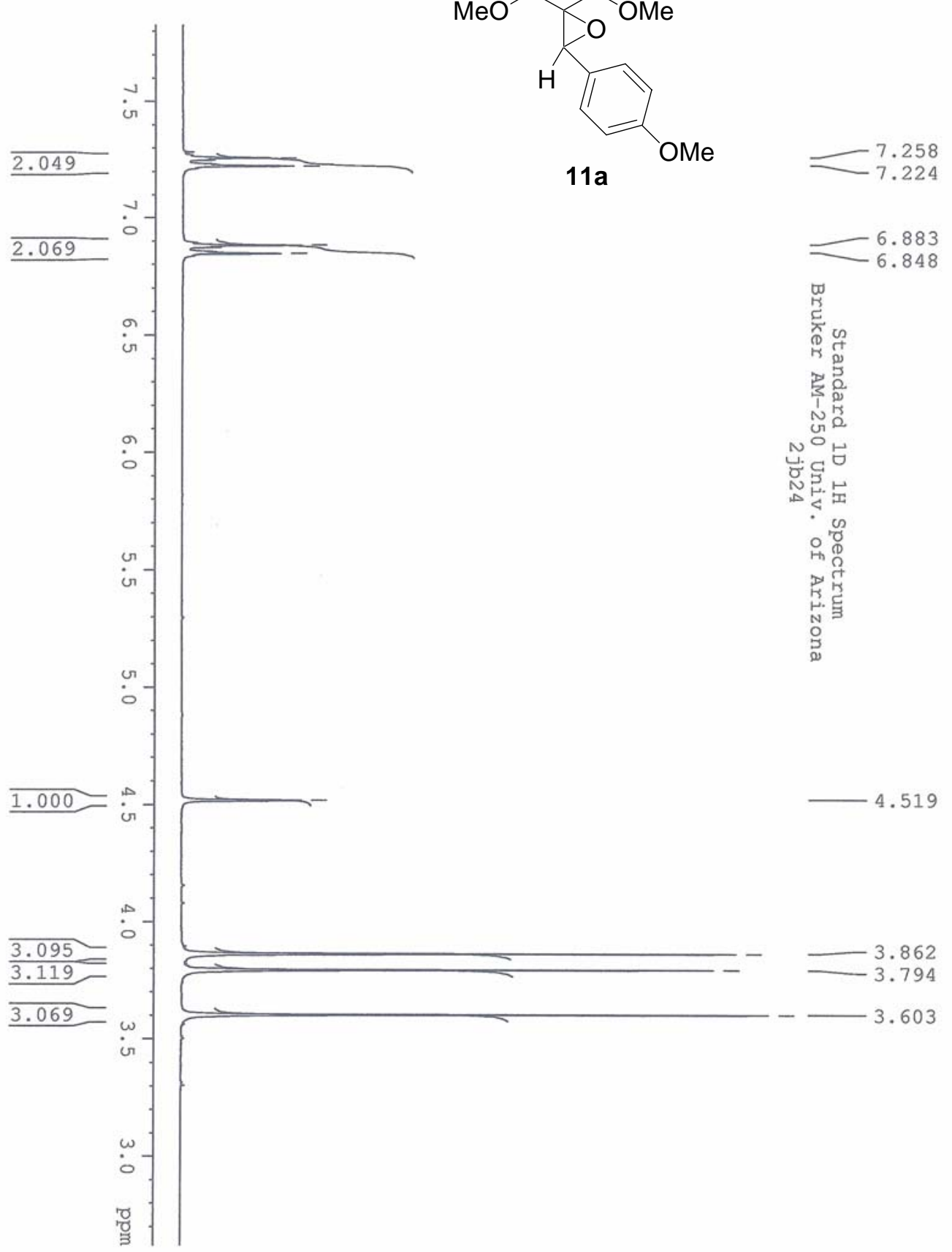




\section{M.P. Doyle et al. - Supporting Information}

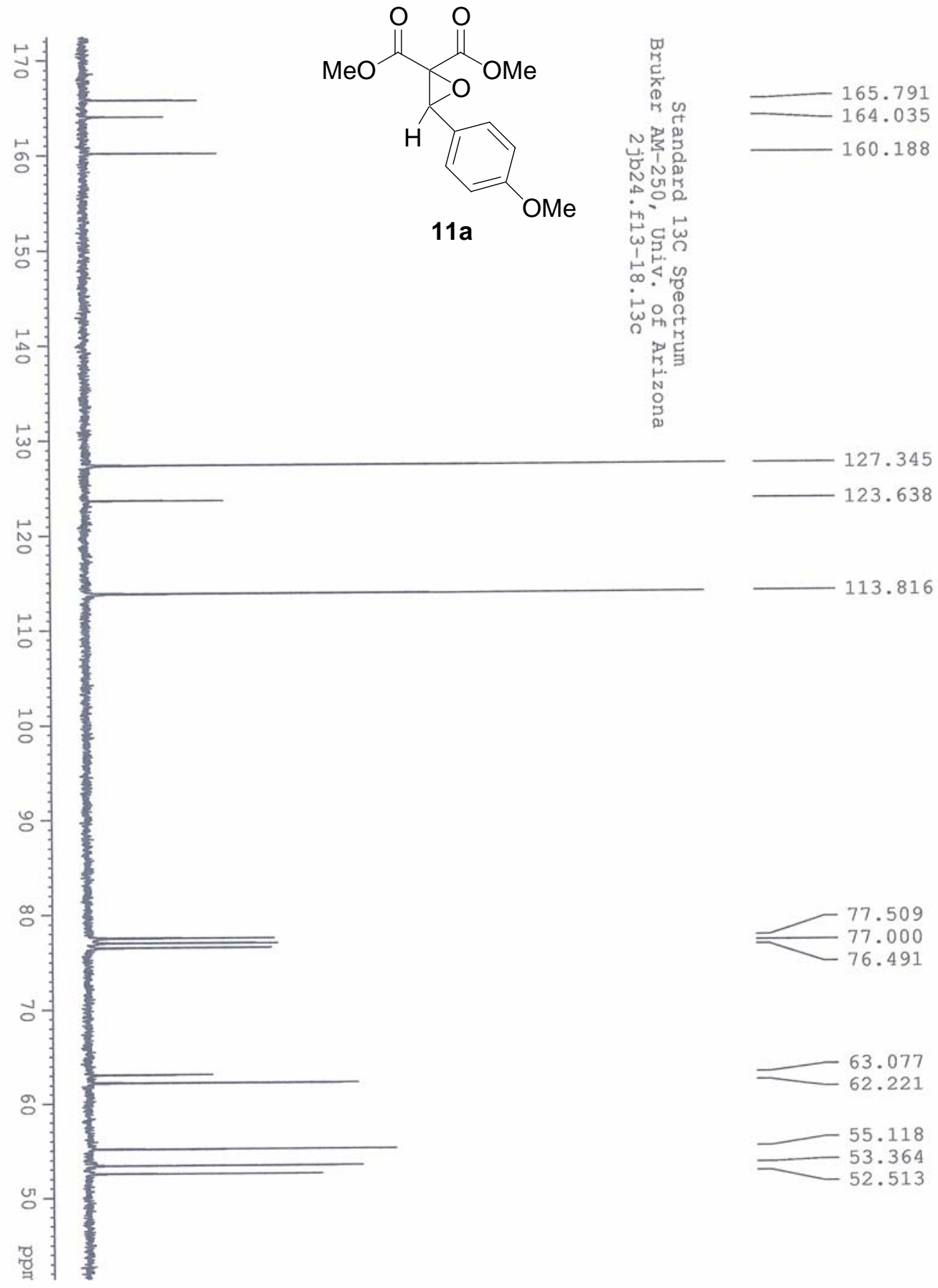




\section{M.P. Doyle et al. - Supporting Information}
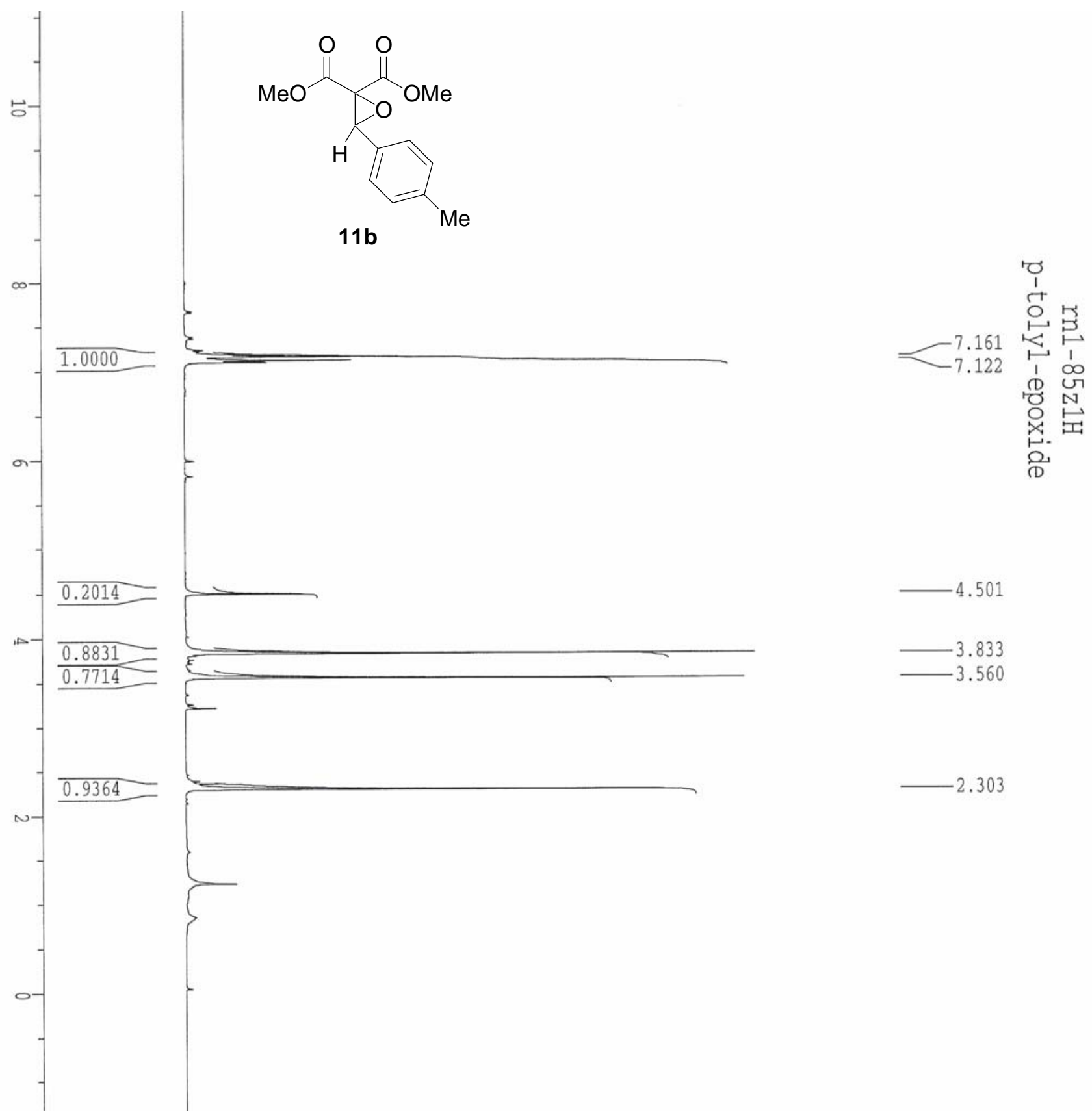

4.501

3.833 


\section{M.P. Doyle et al. - Supporting Information}

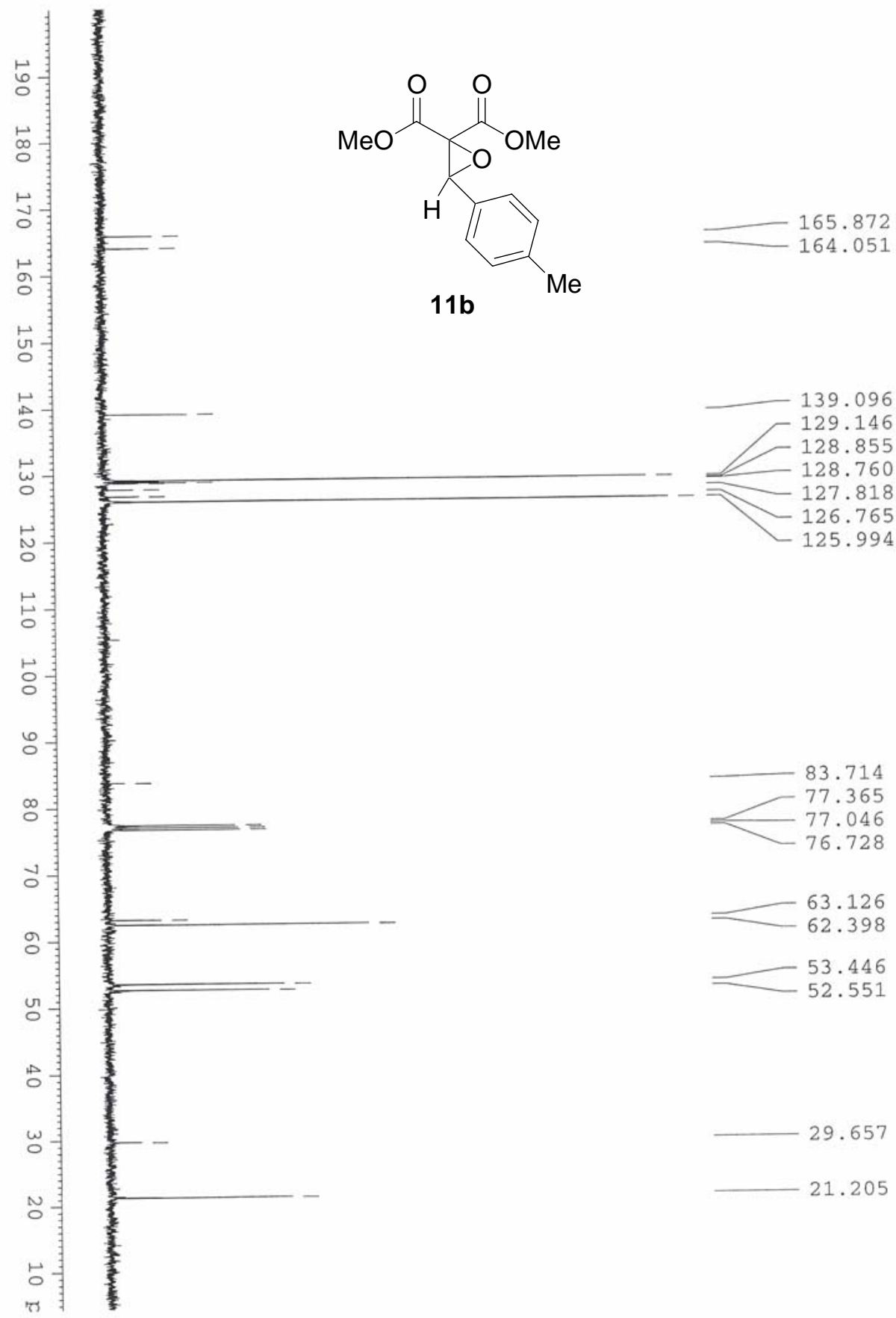




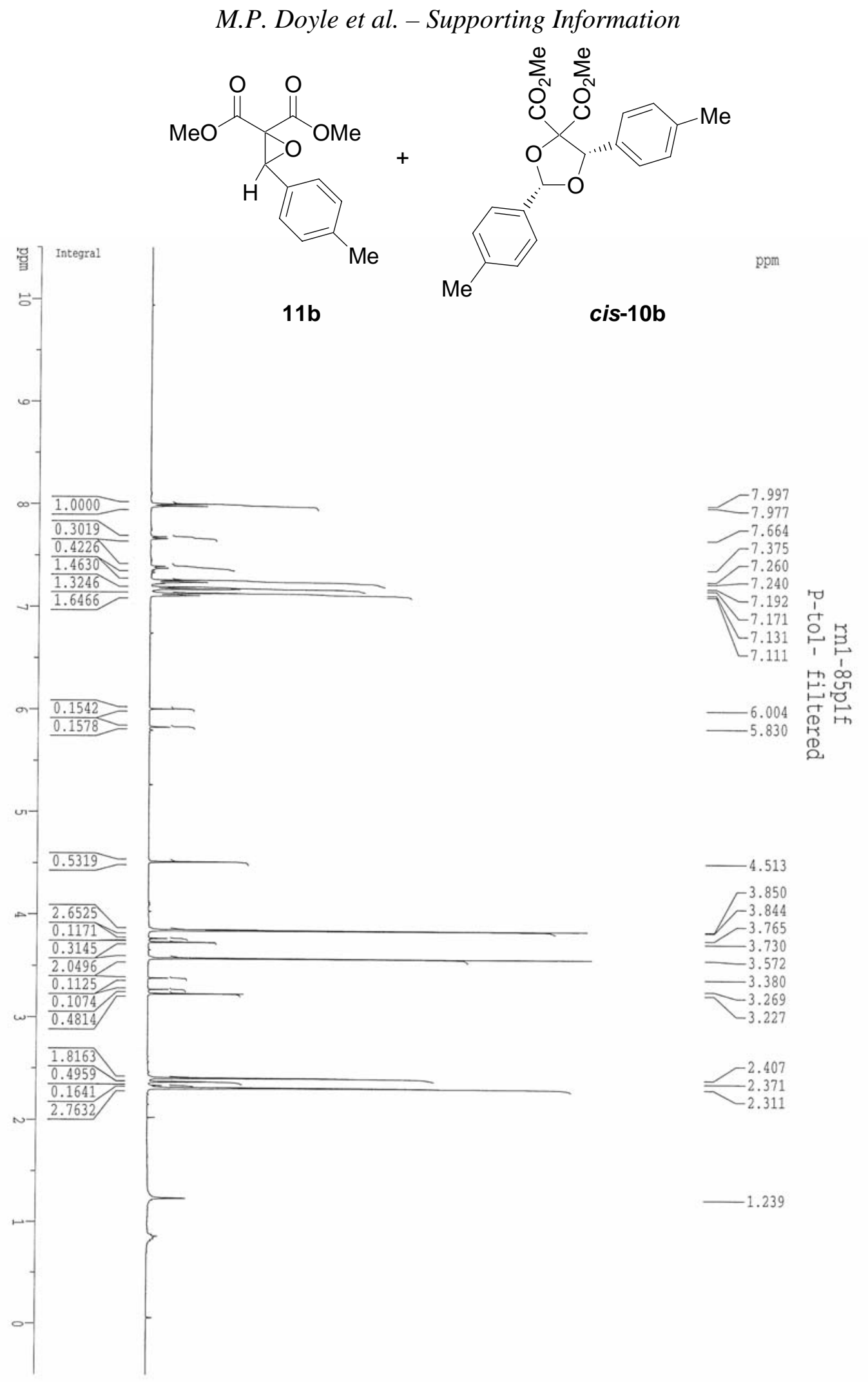


M.P. Doyle et al. - Supporting Information

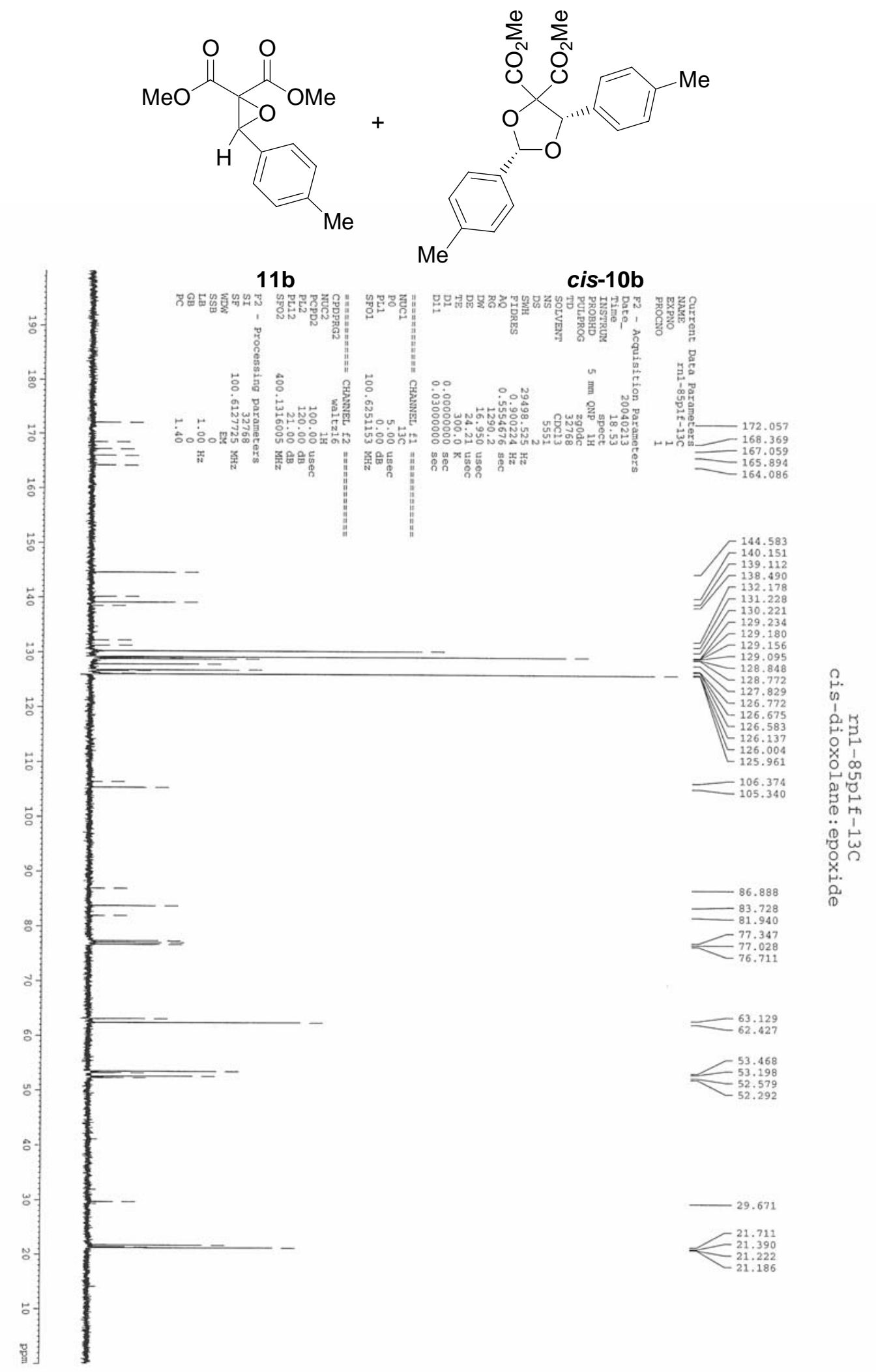


M.P. Doyle et al. - Supporting Information

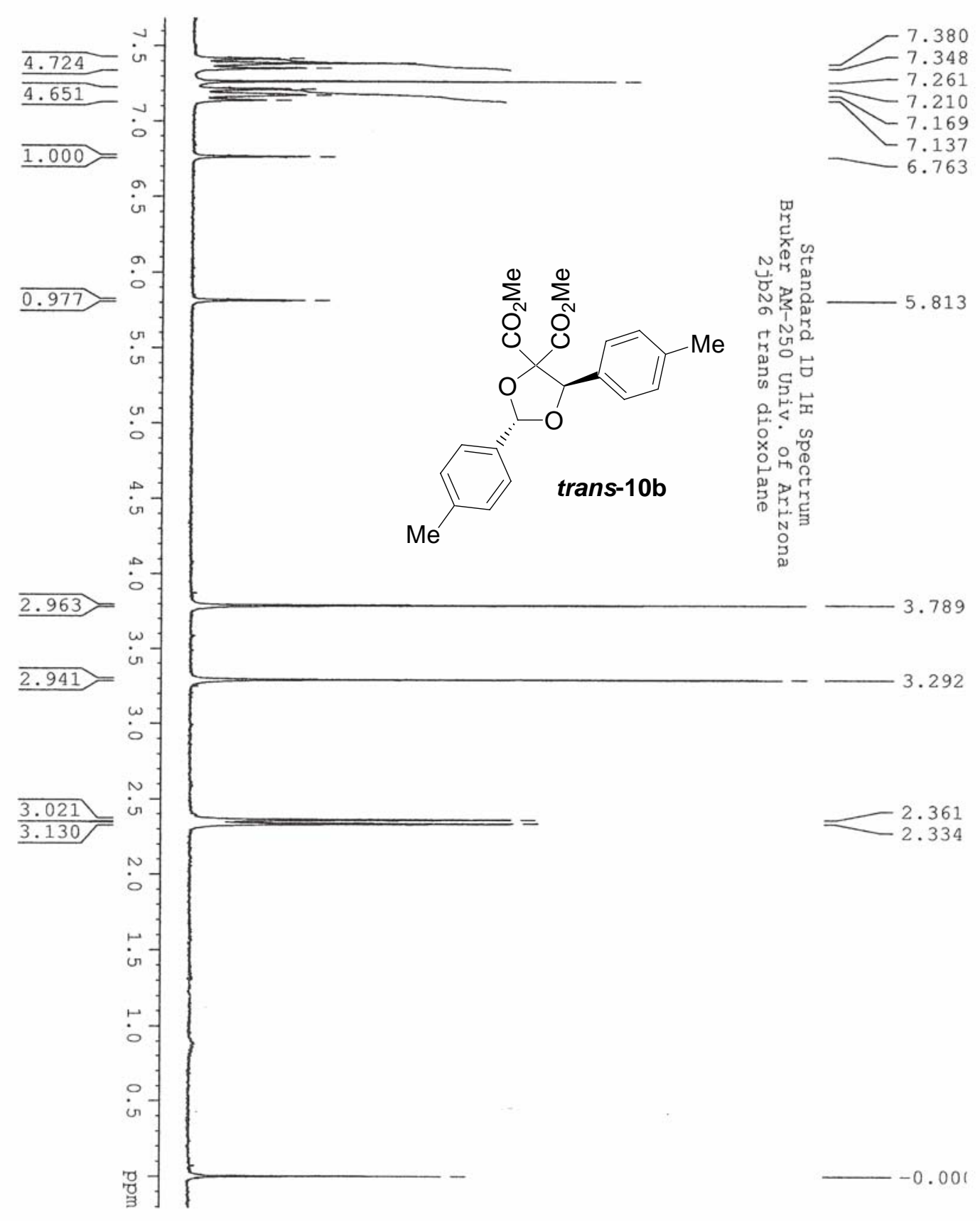




\section{M.P. Doyle et al. - Supporting Information}

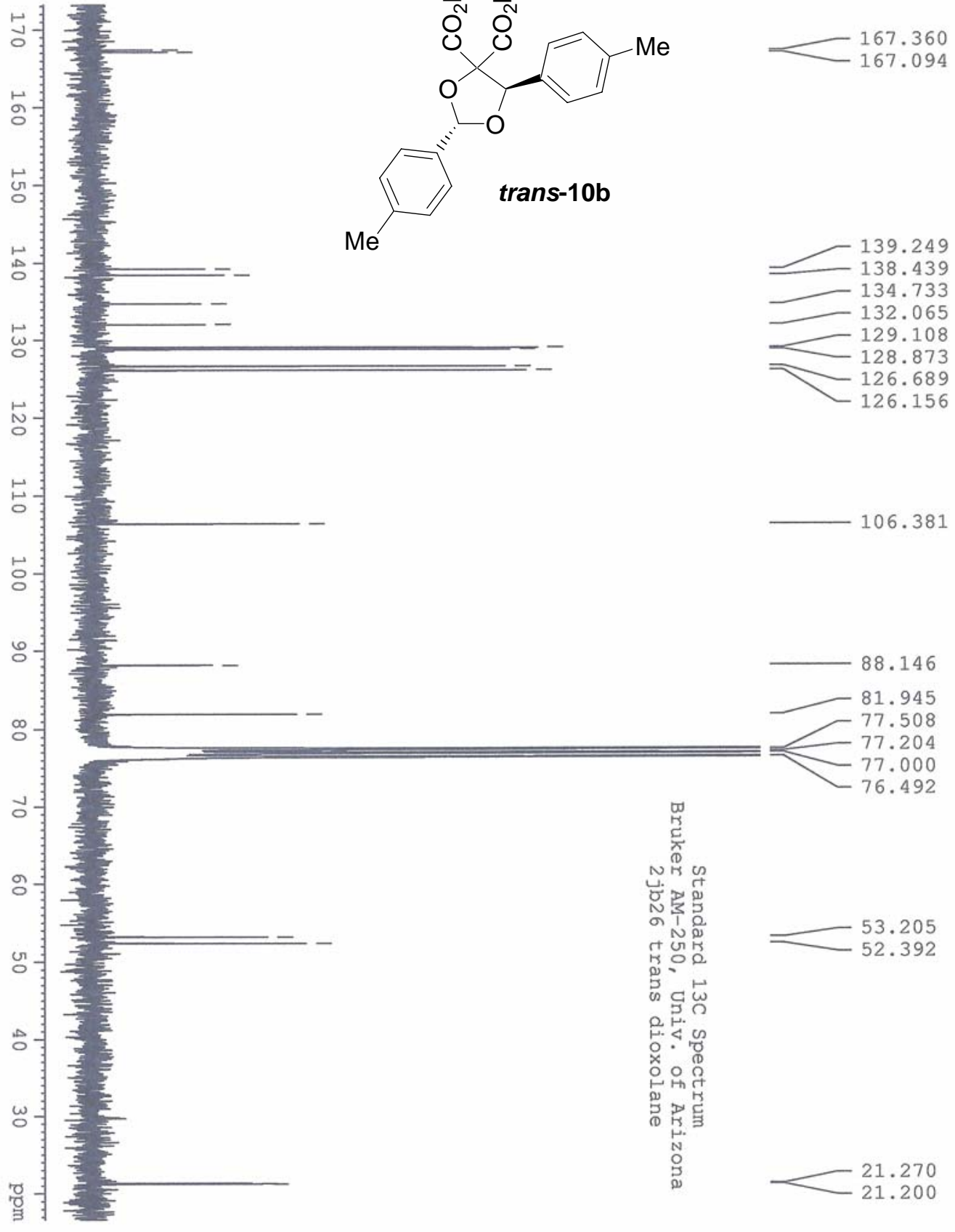


M.P. Doyle et al. - Supporting Information

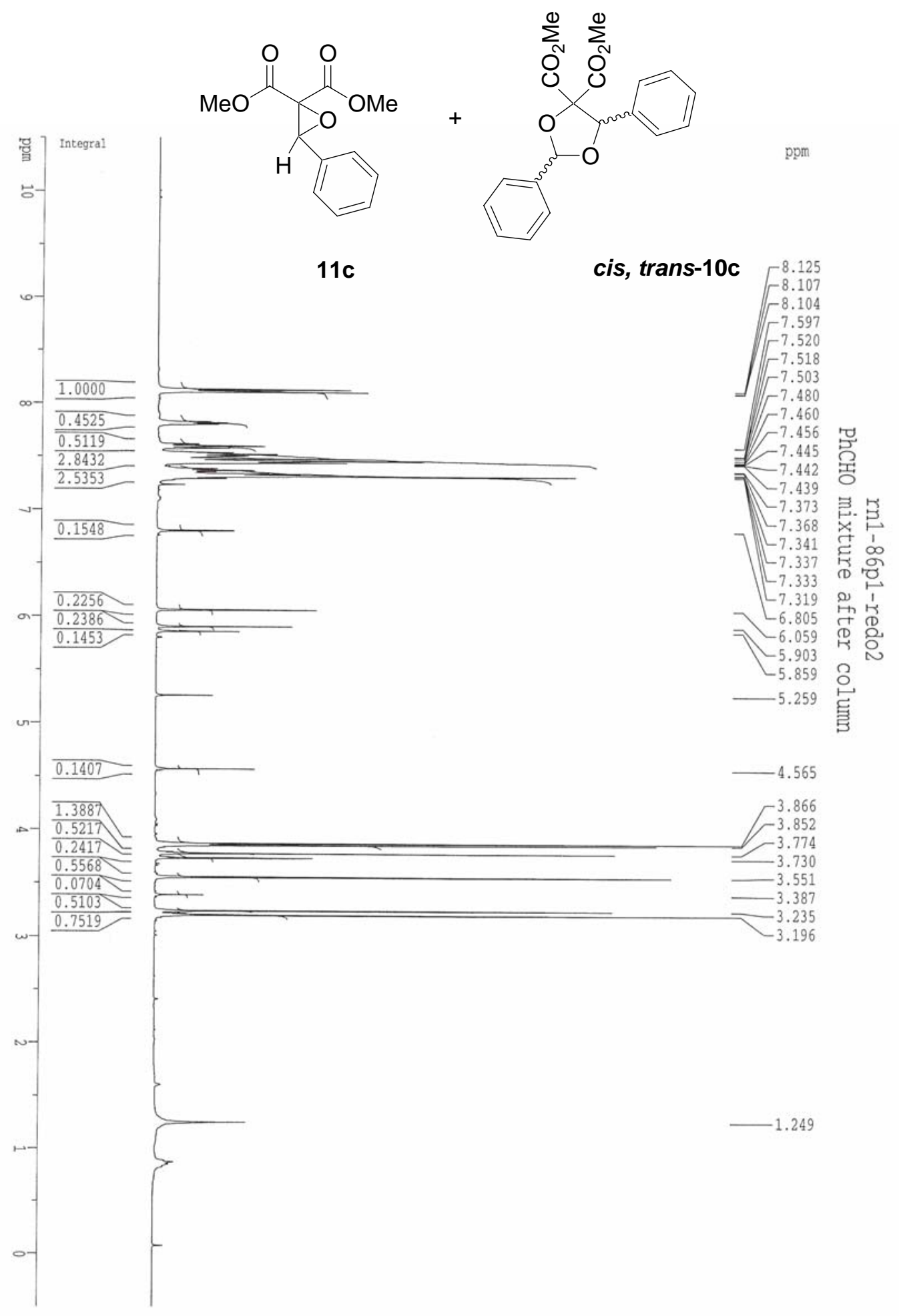


M.P. Doyle et al. - Supporting Information

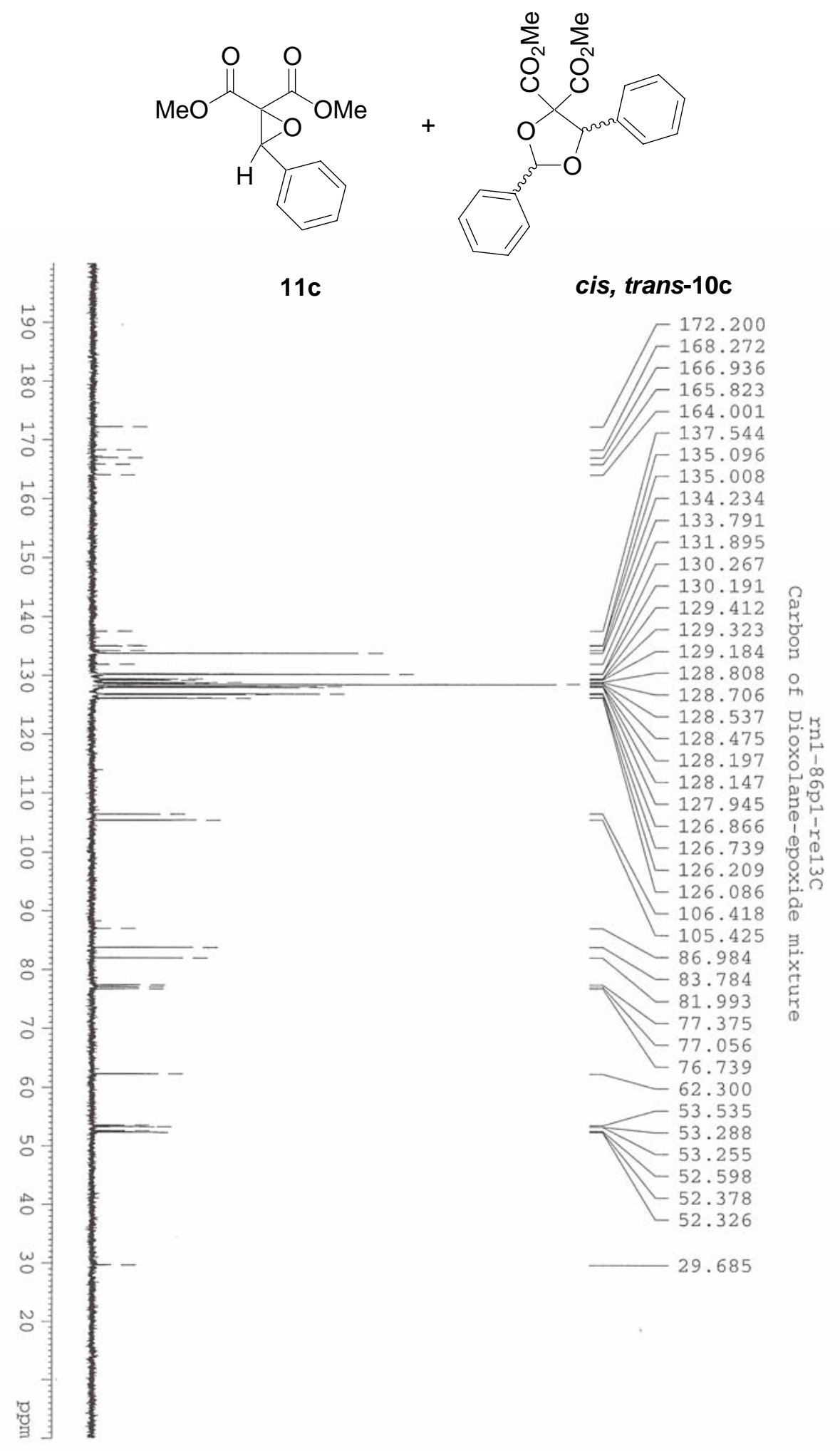


M.P. Doyle et al. - Supporting Information
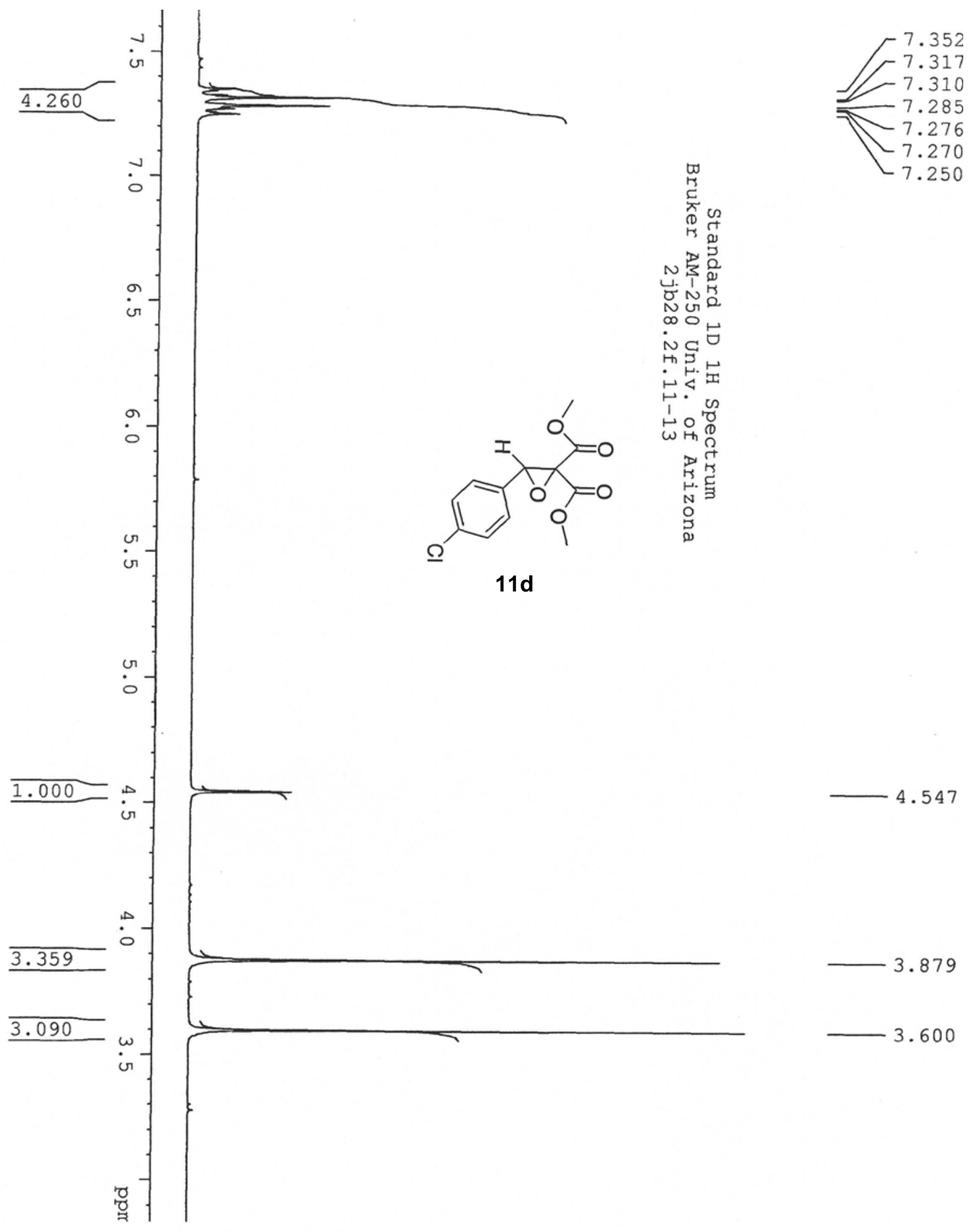

$-3.879$

3.600 


\section{M.P. Doyle et al. - Supporting Information}
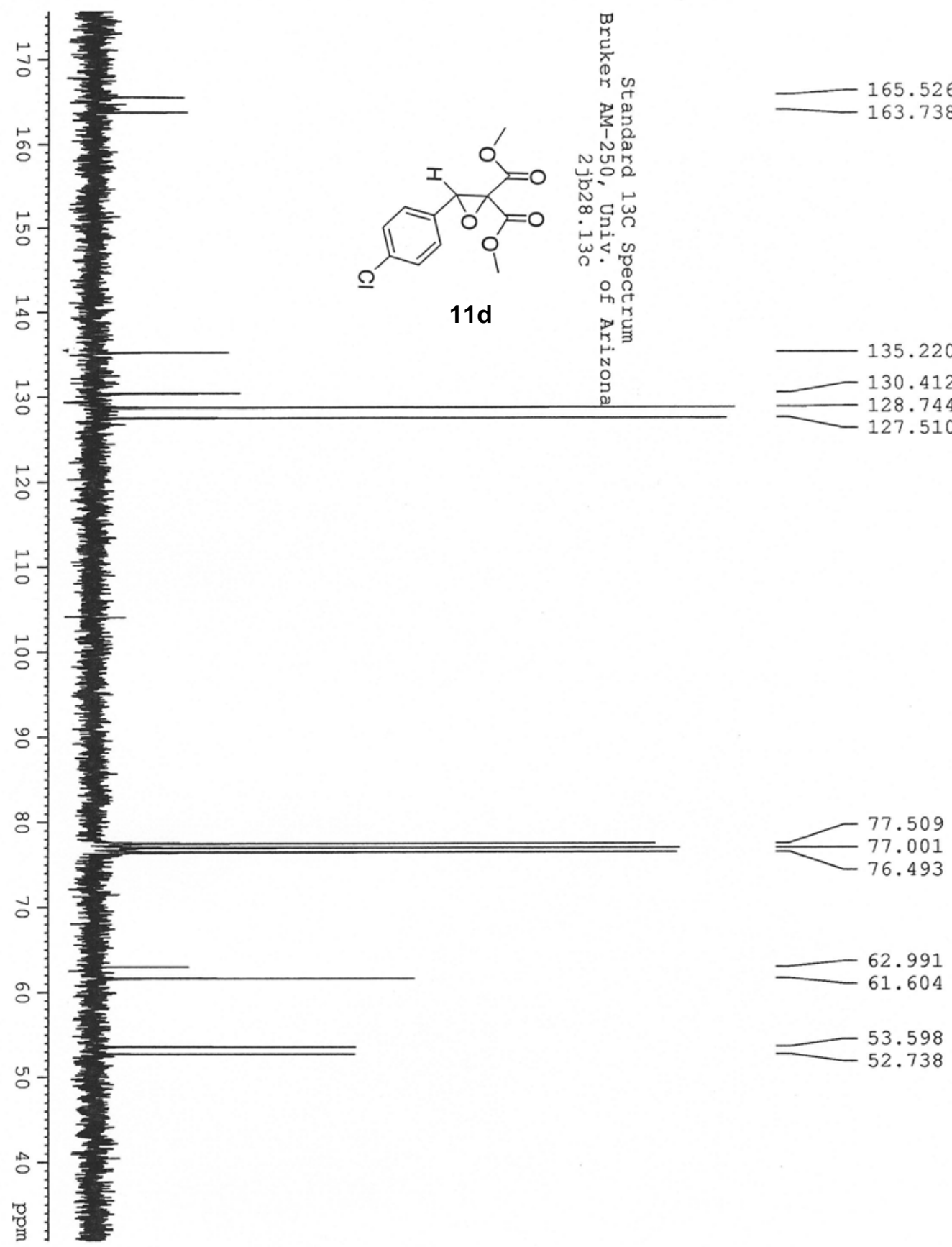
M.P. Doyle et al. - Supporting Information

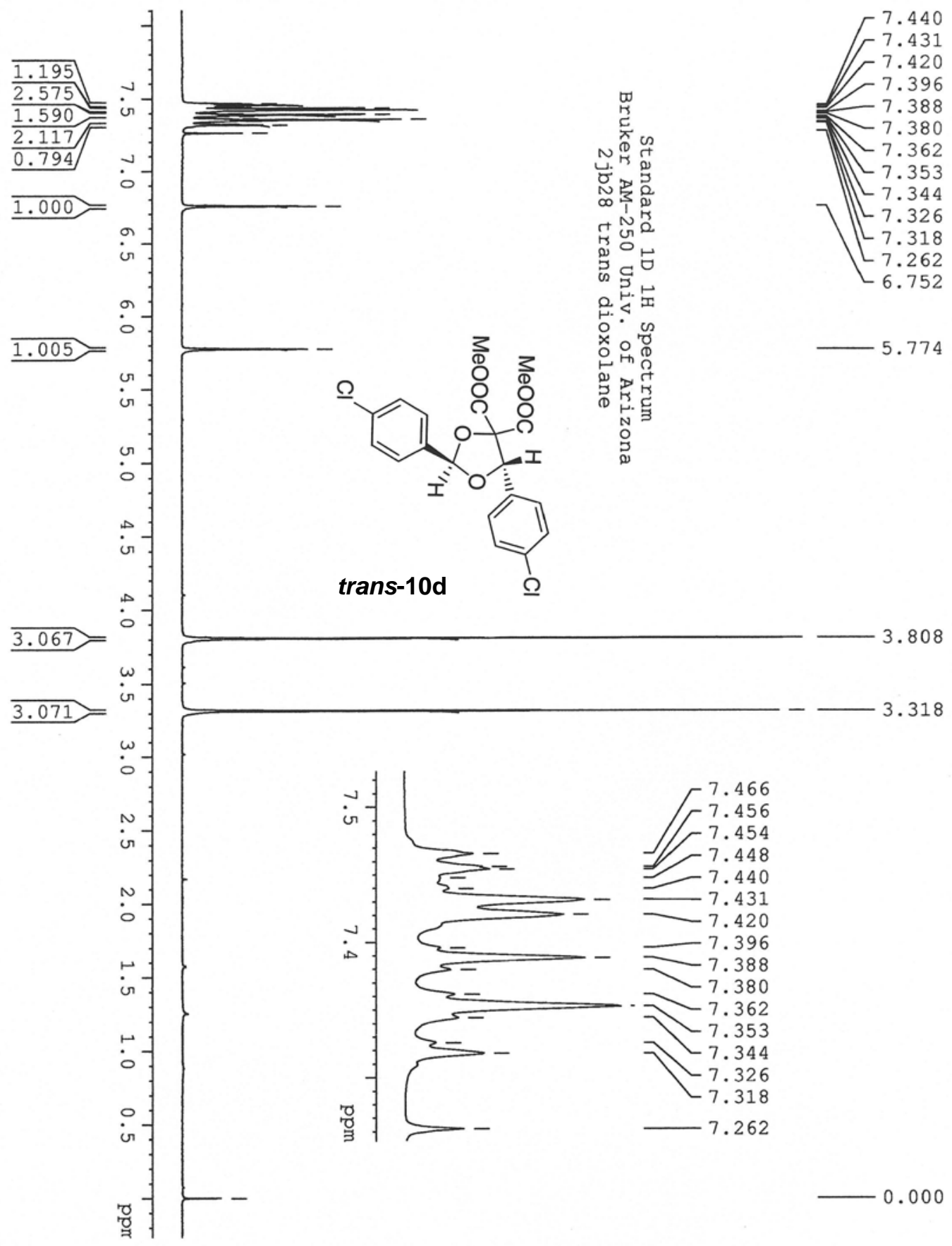




\section{M.P. Doyle et al. - Supporting Information}

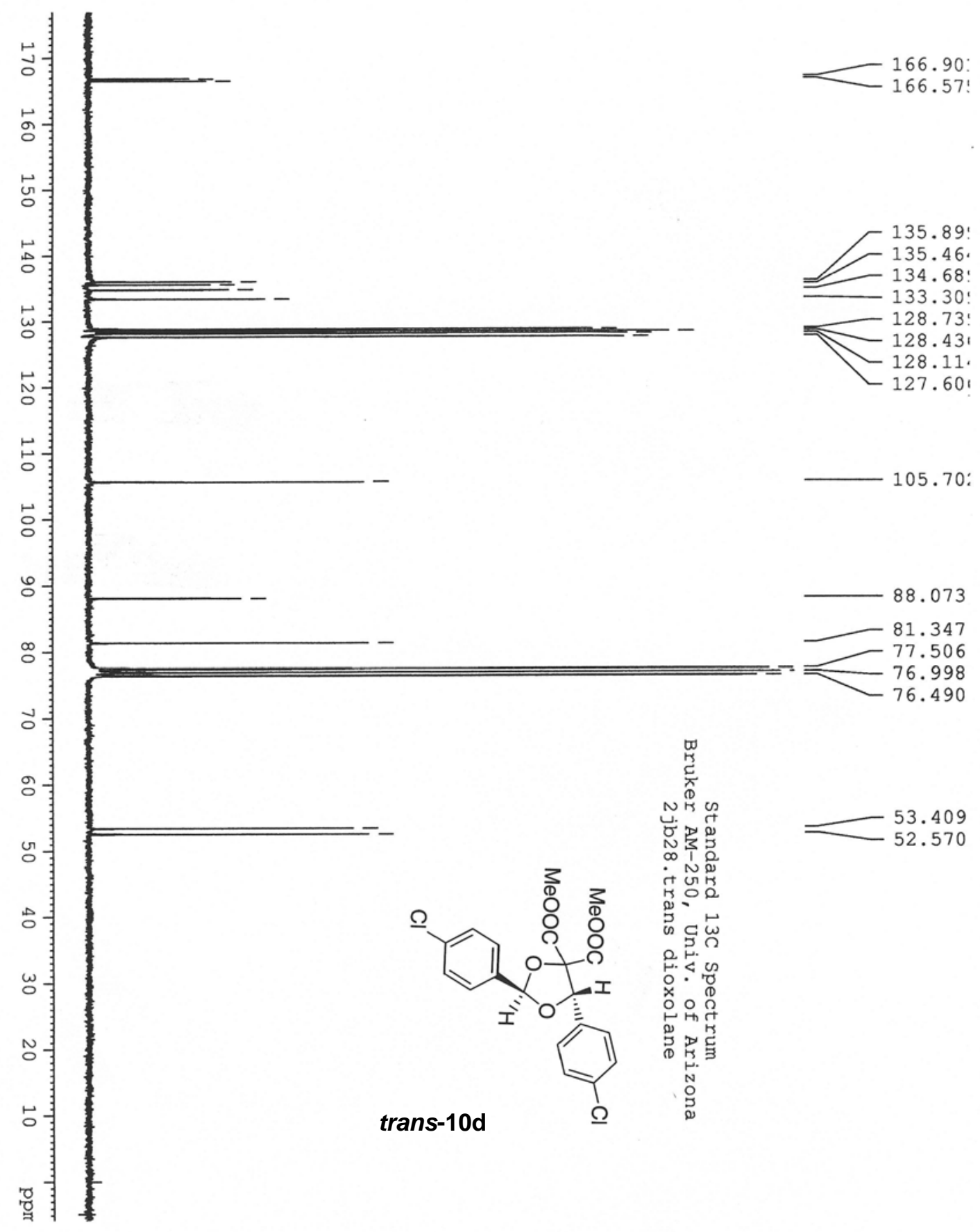




\section{M.P. Doyle et al. - Supporting Information}

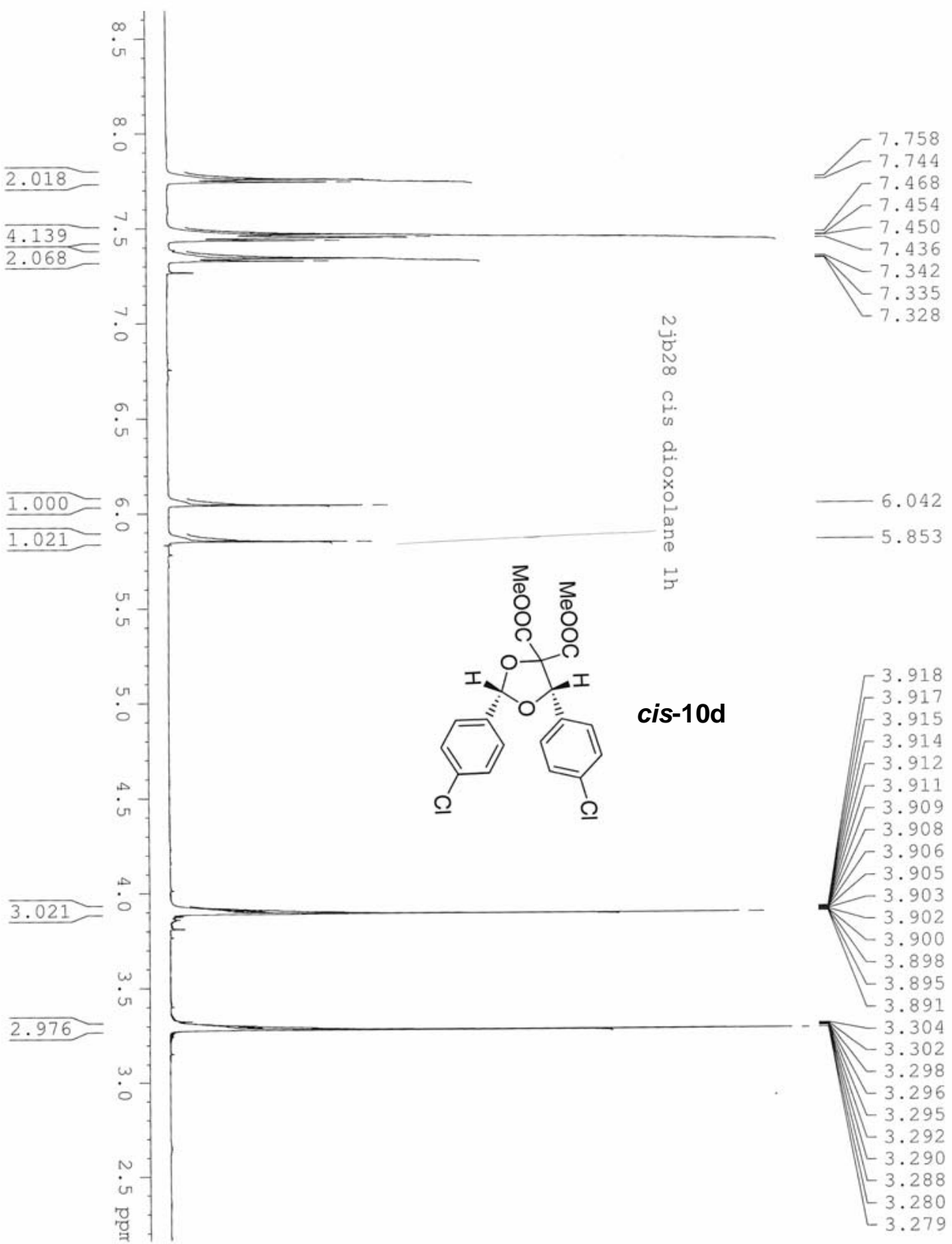




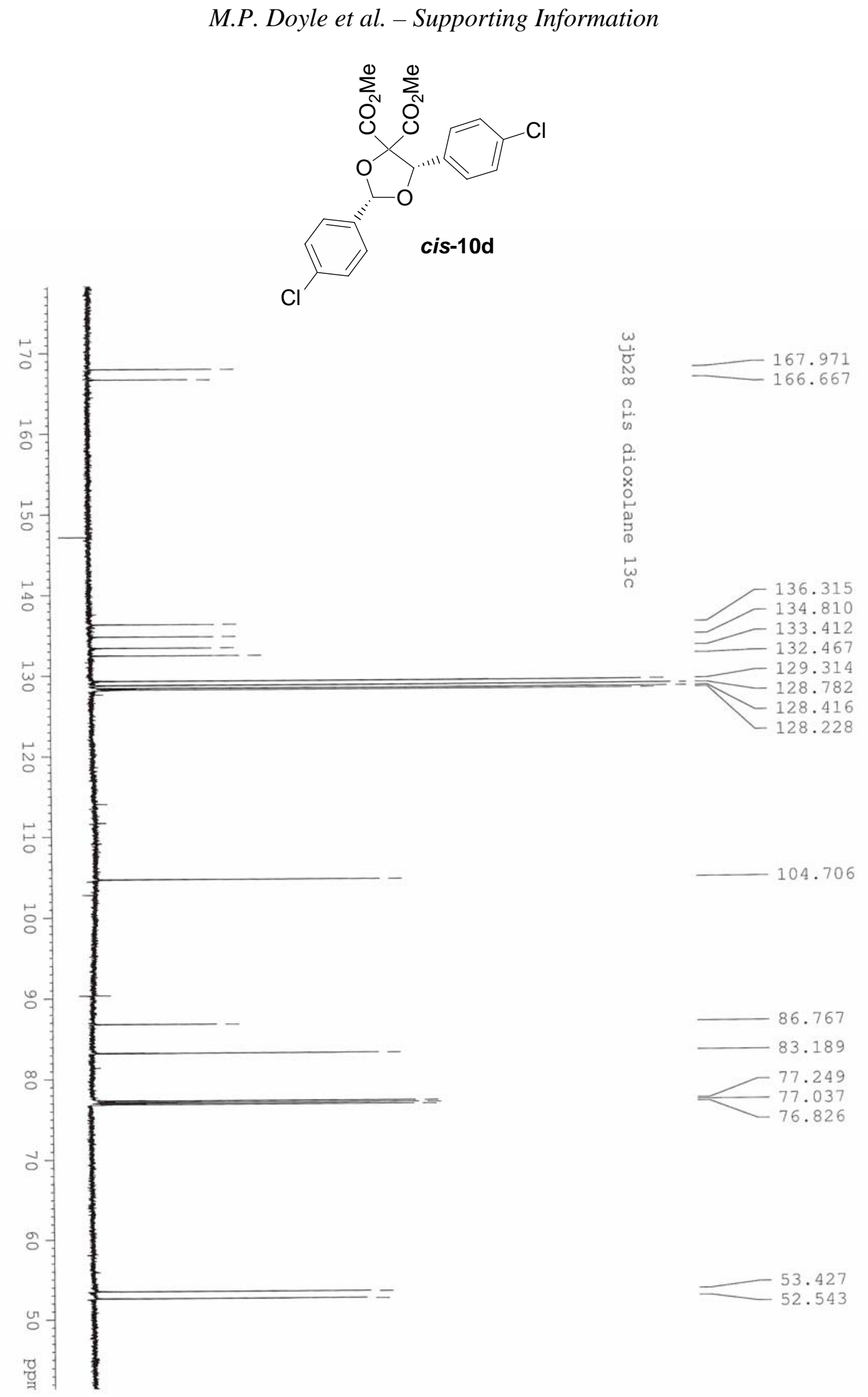




\section{M.P. Doyle et al. - Supporting Information}

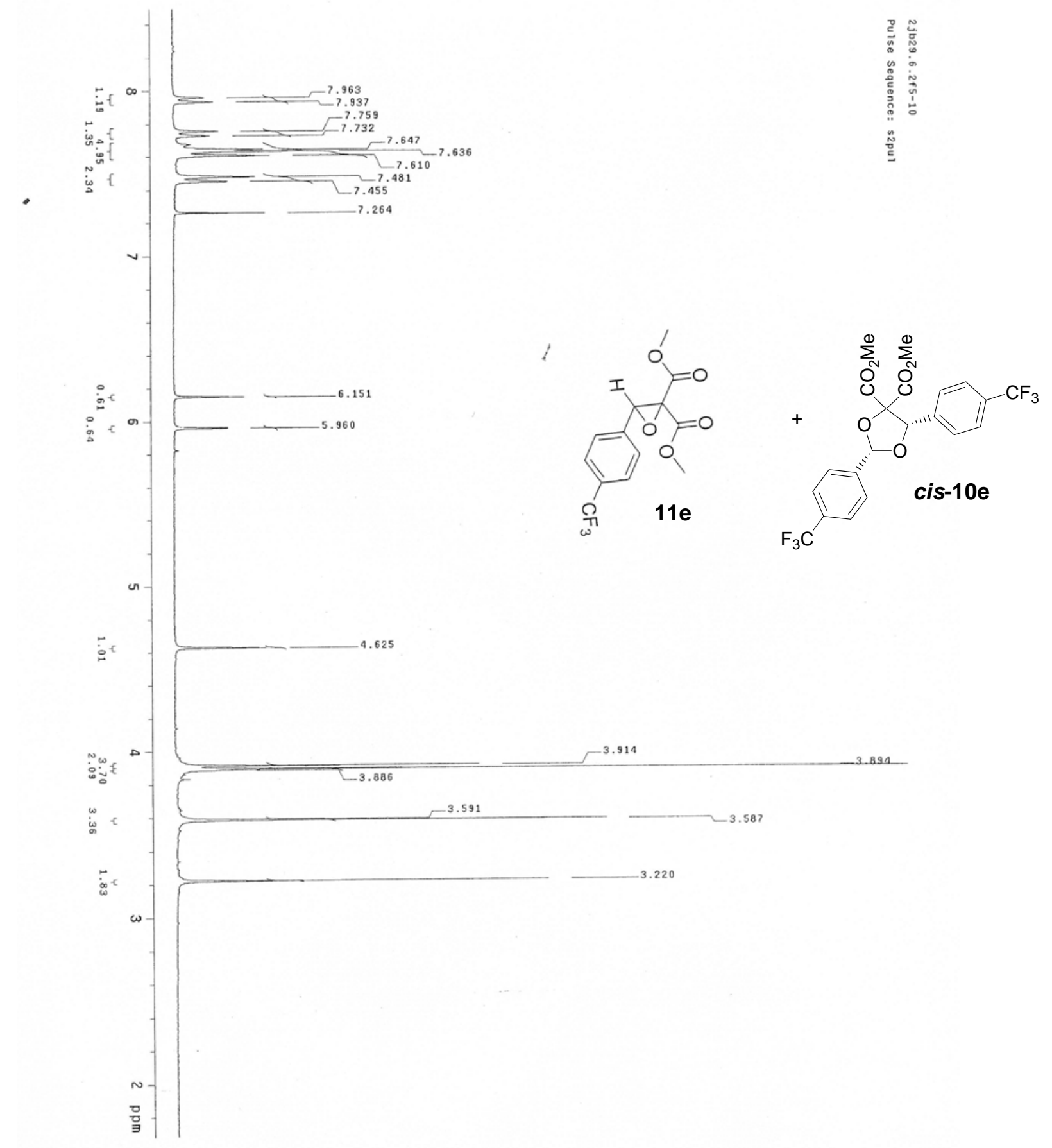


M.P. Doyle et al. - Supporting Information

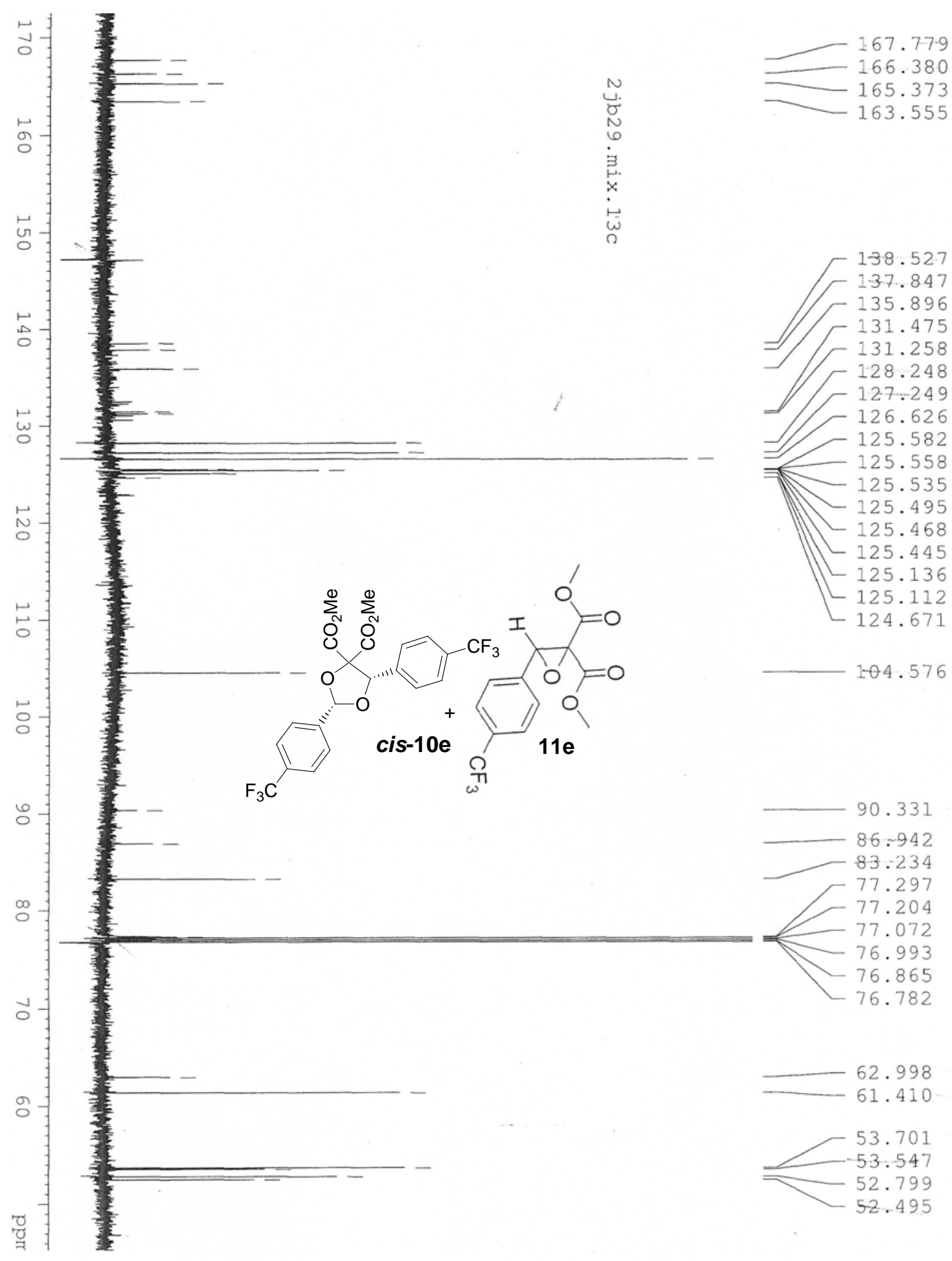


M.P. Doyle et al. - Supporting Information

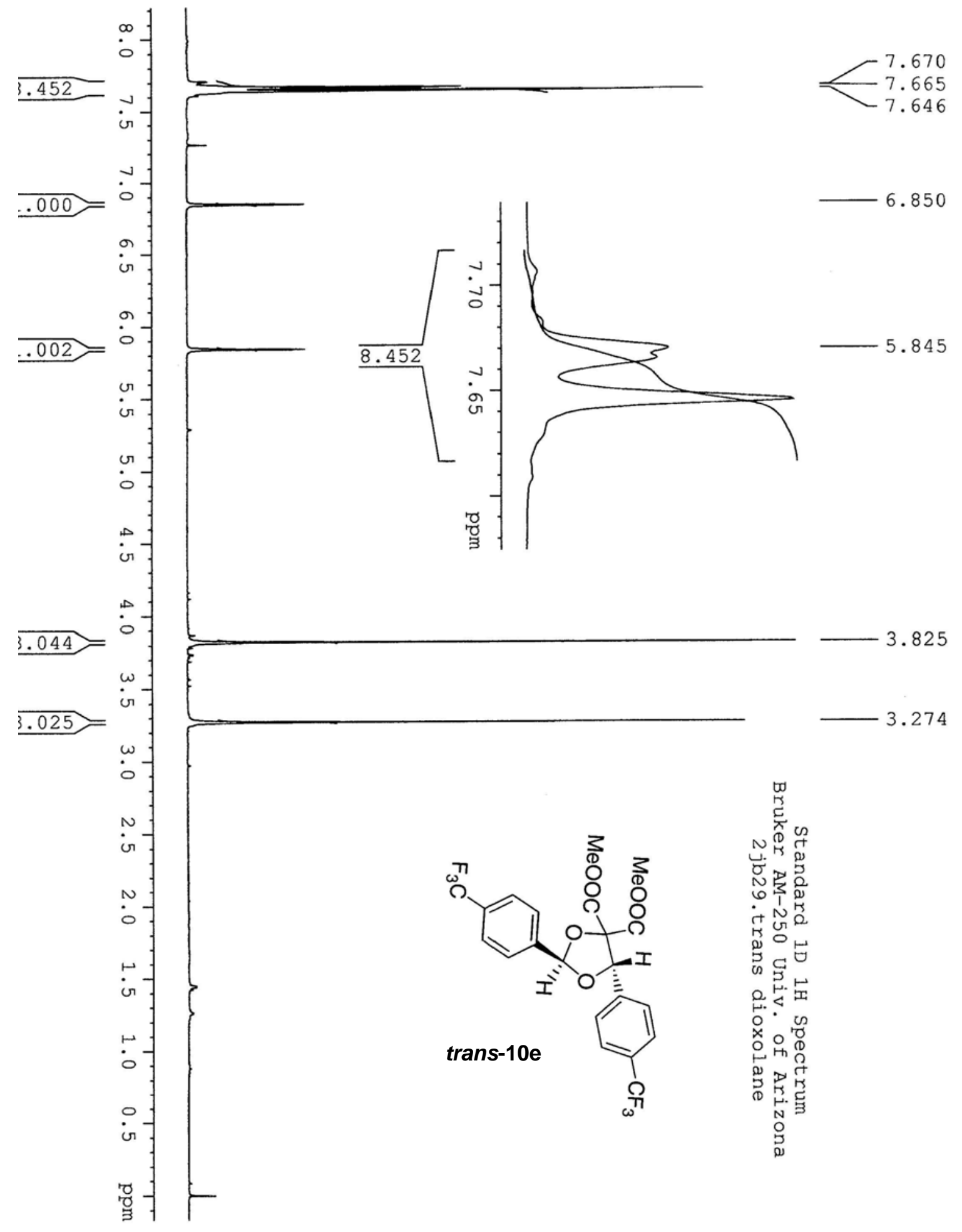


M.P. Doyle et al. - Supporting Information

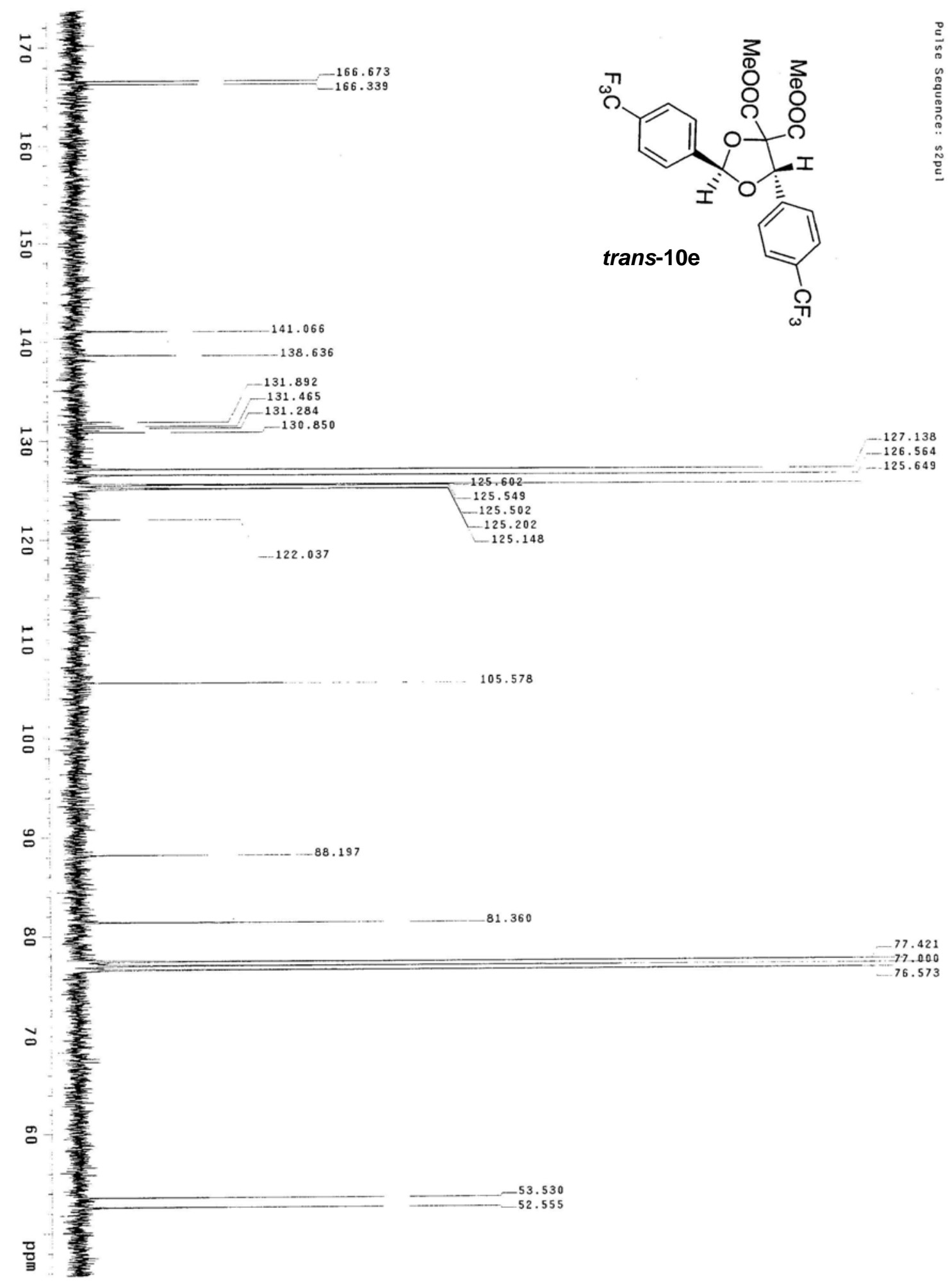




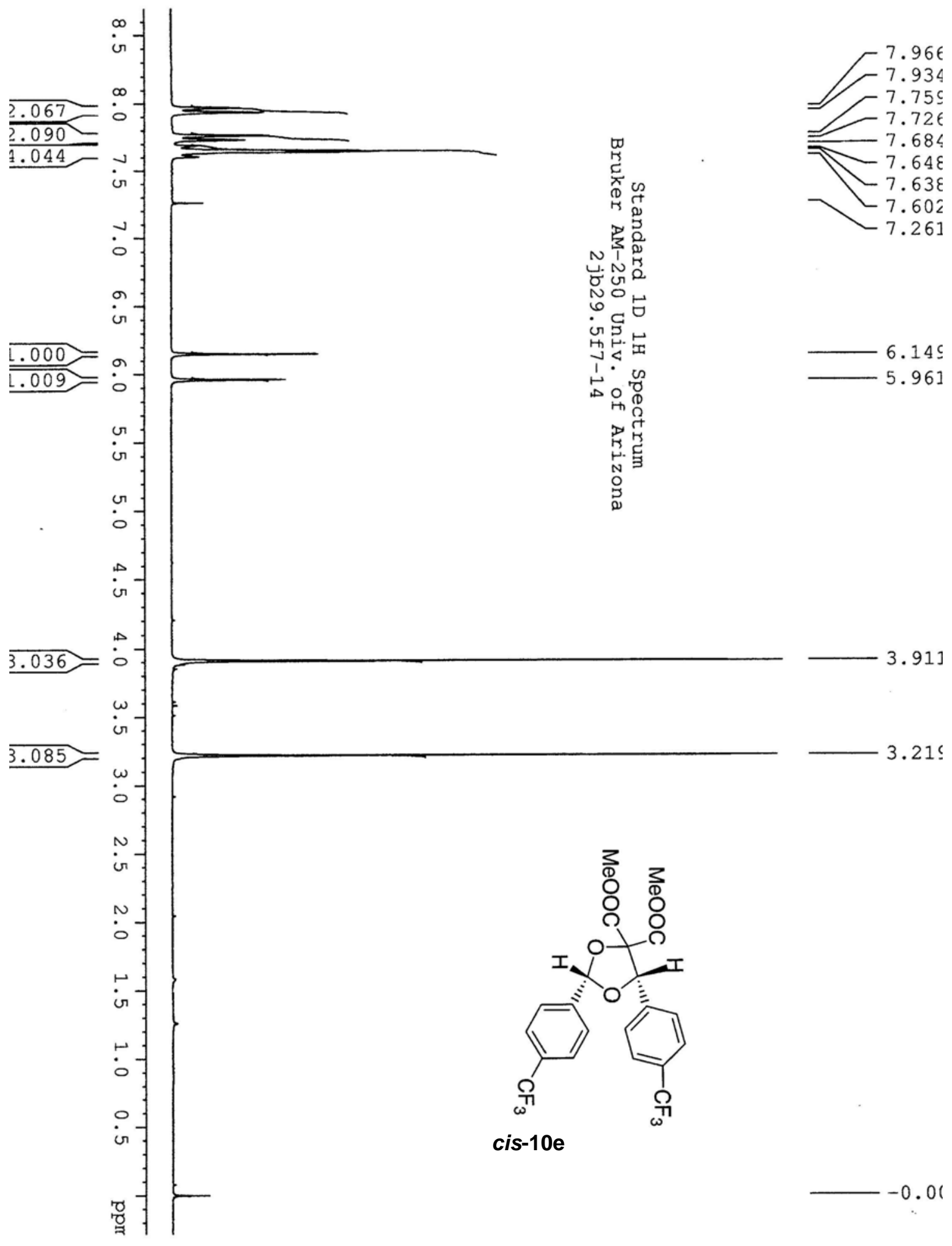




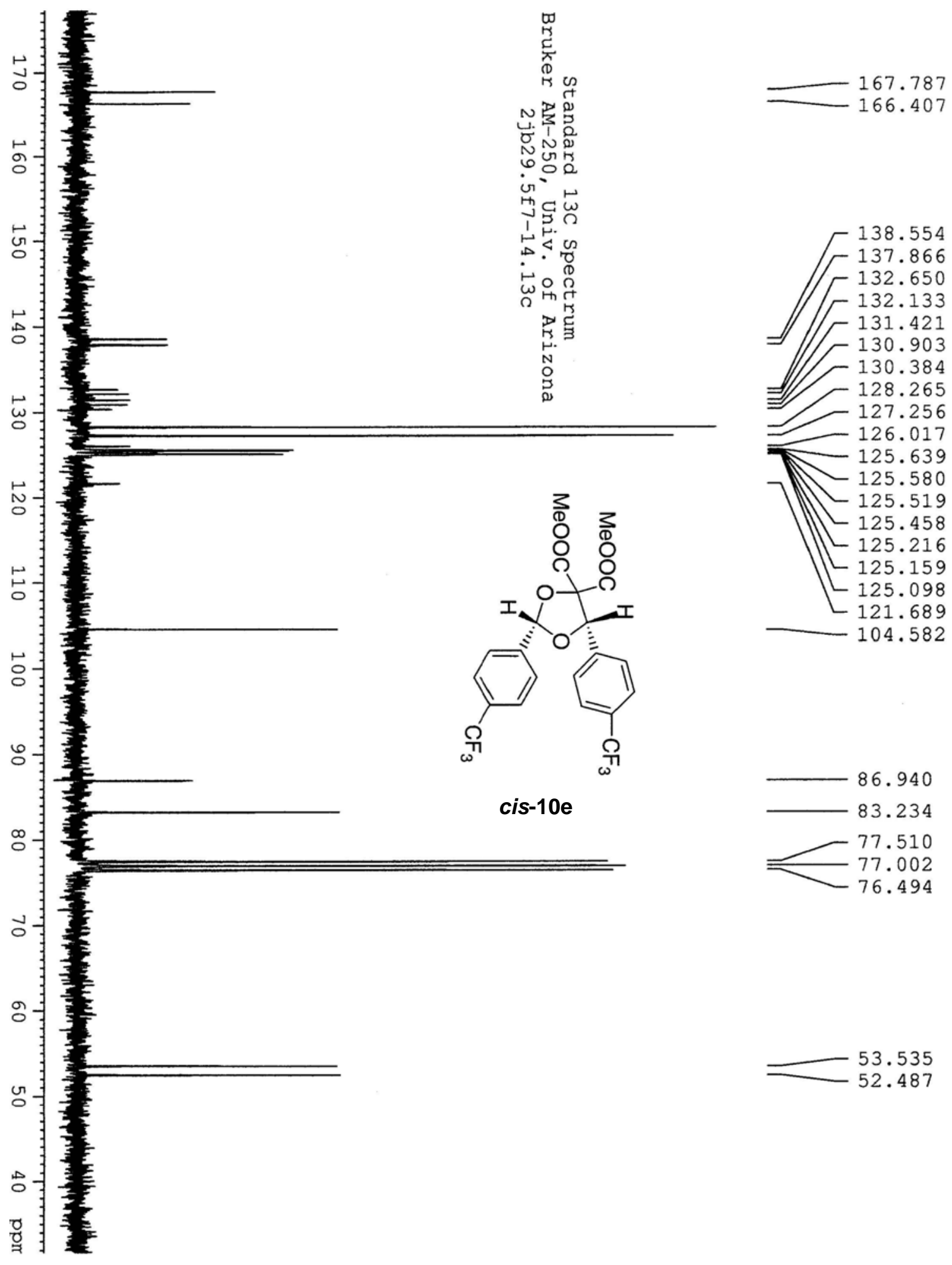


M.P. Doyle et al. - Supporting Information
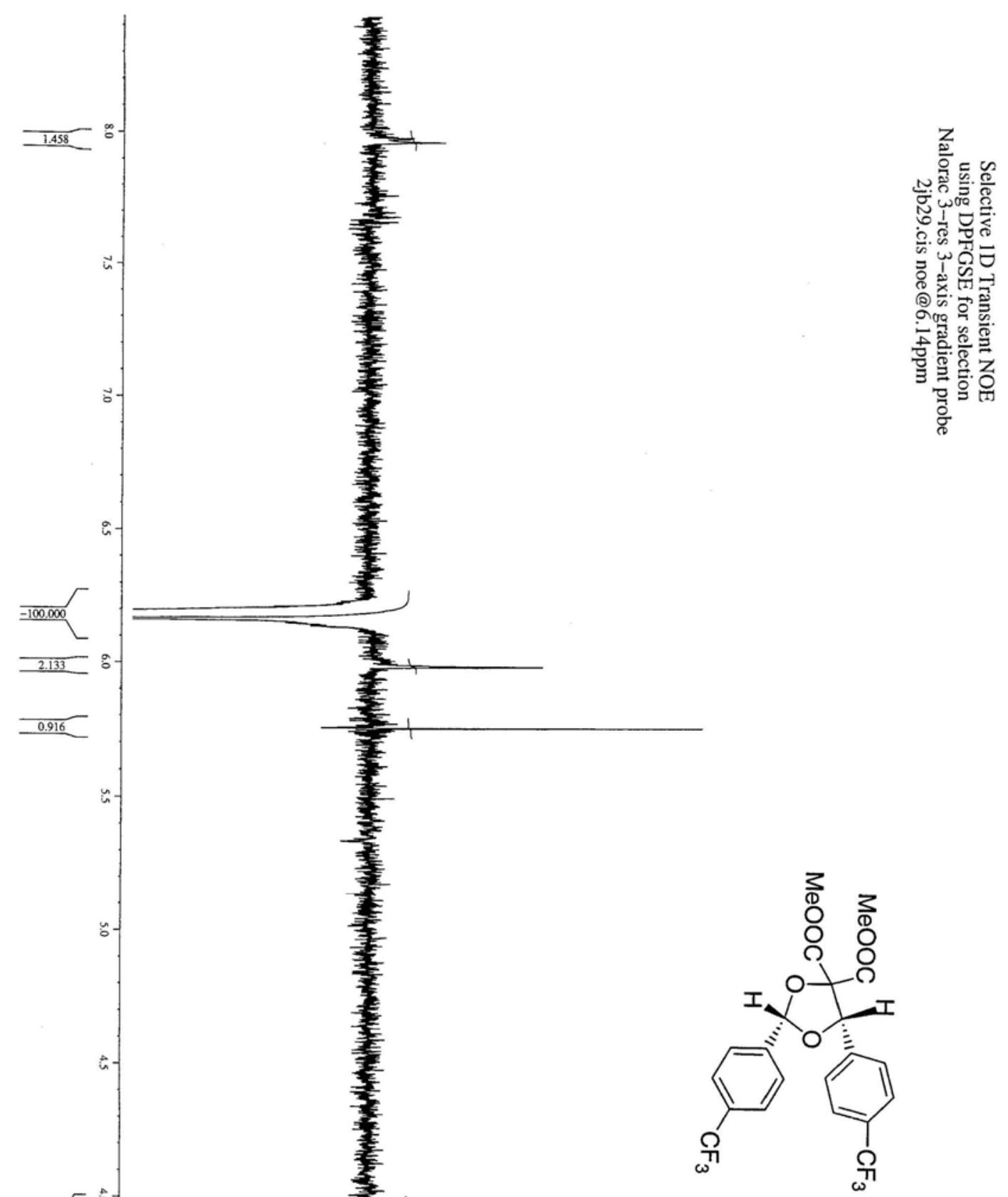

cis-10e 


\section{M.P. Doyle et al. - Supporting Information}
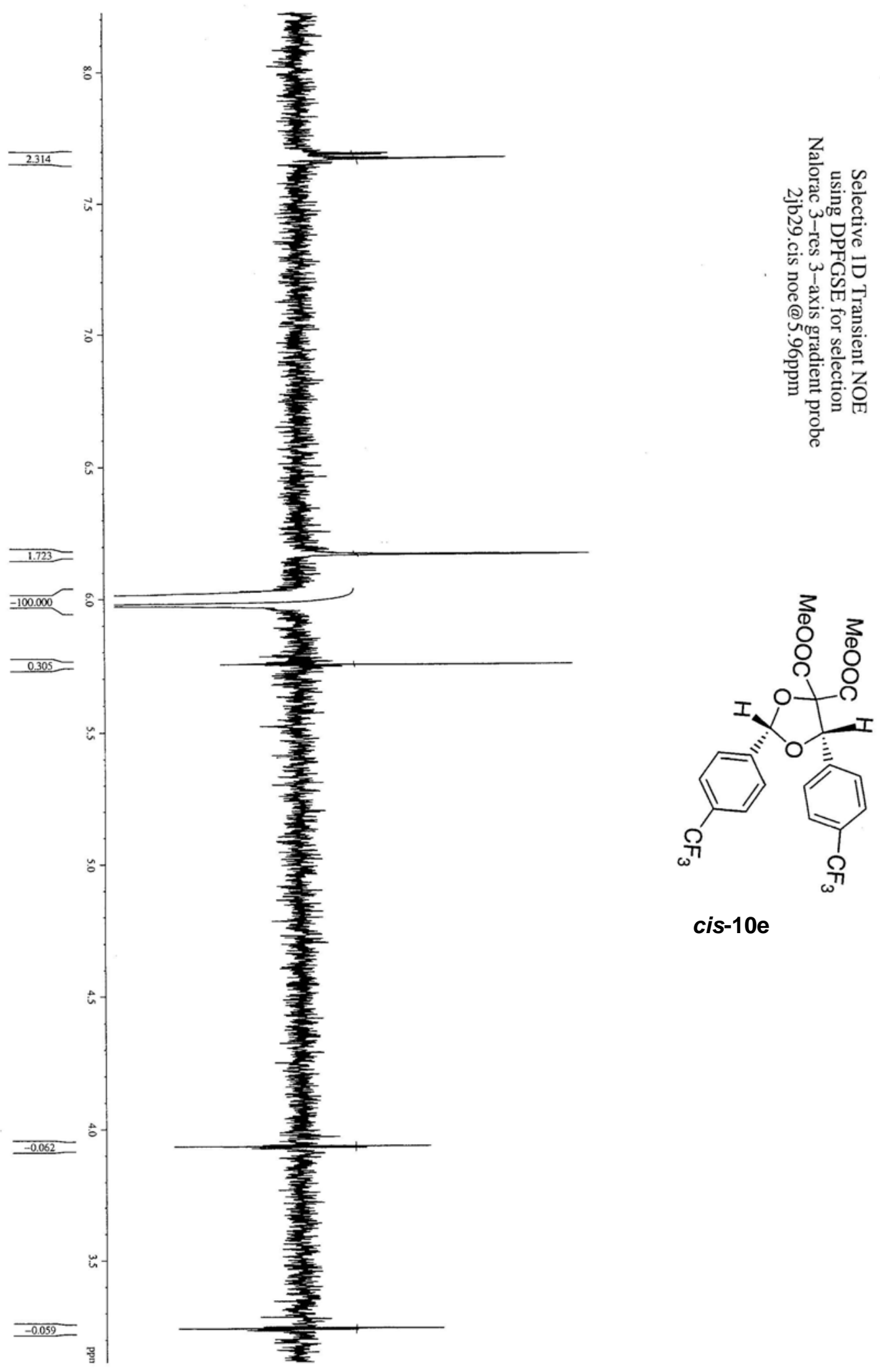

cis-10e 


\section{M.P. Doyle et al. - Supporting Information}

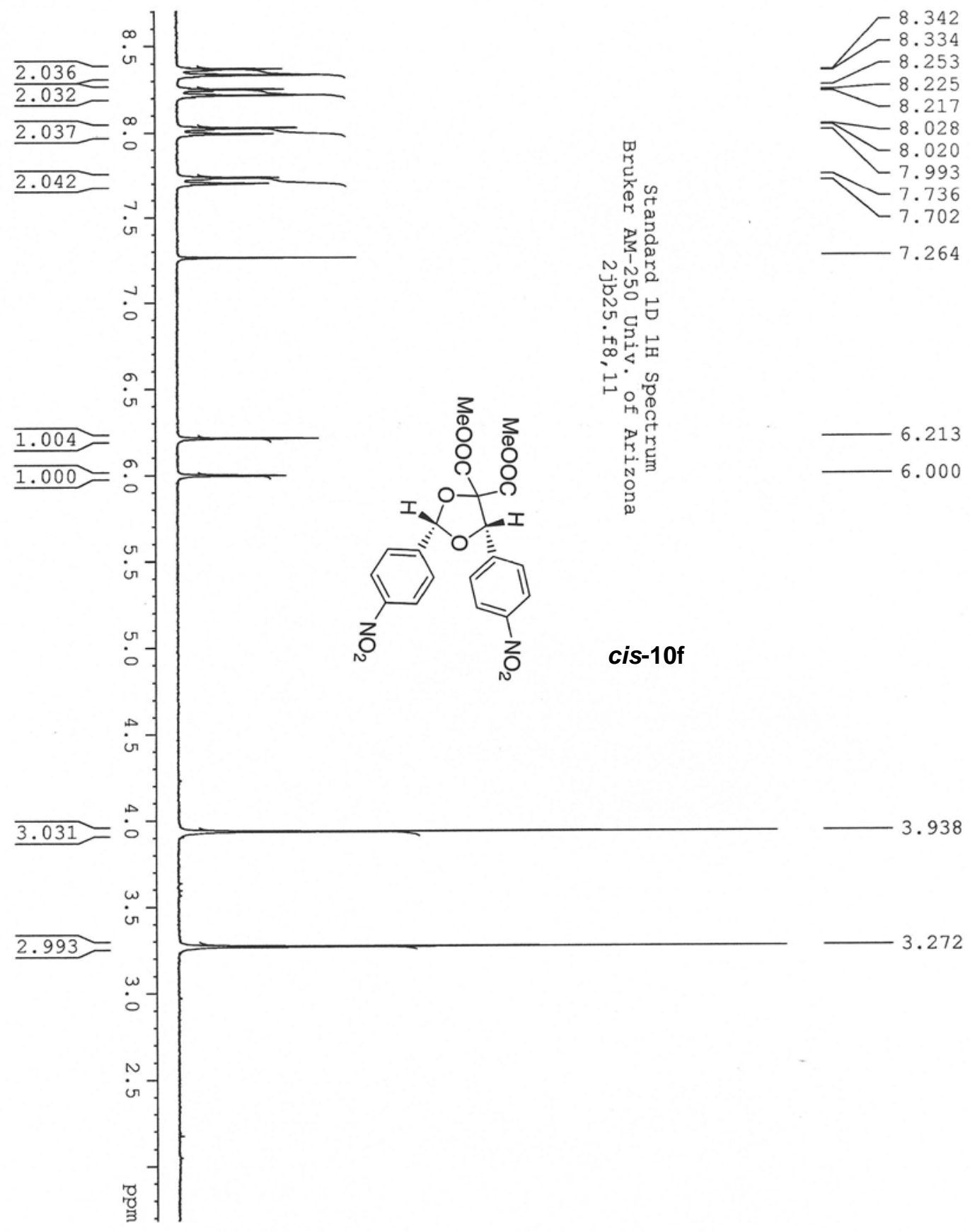




\section{M.P. Doyle et al. - Supporting Information}

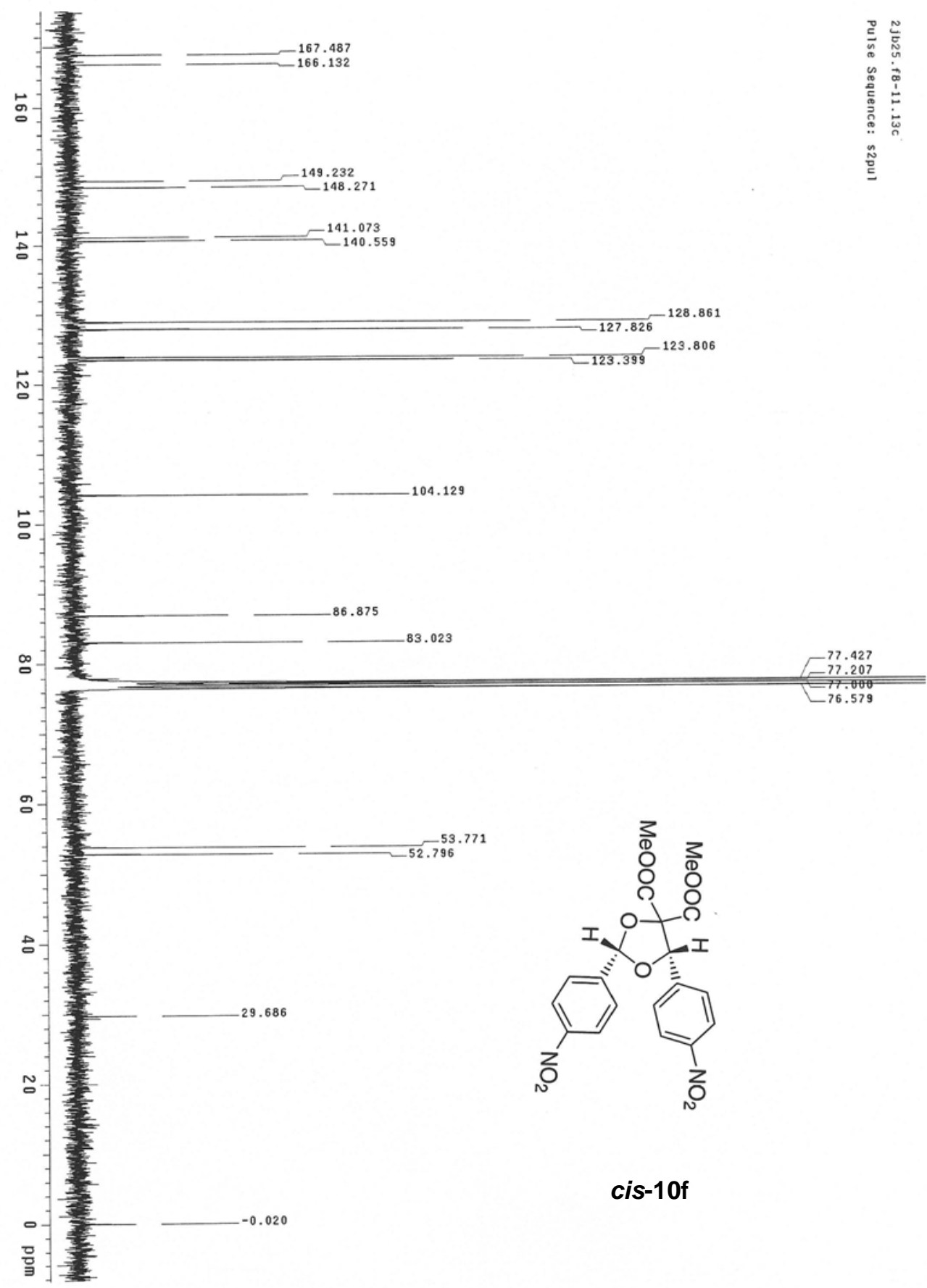


M.P. Doyle et al. - Supporting Information

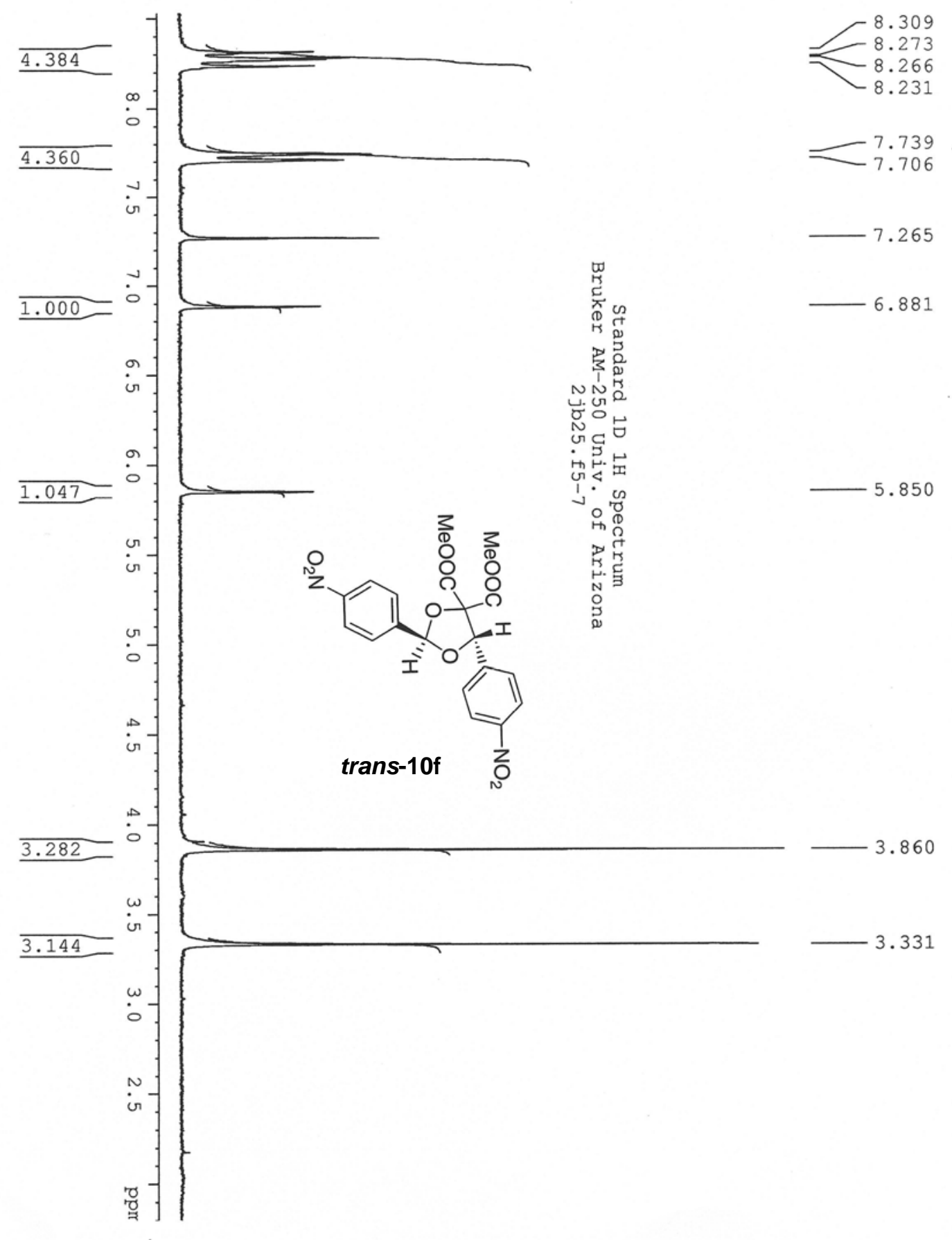


M.P. Doyle et al. - Supporting Information

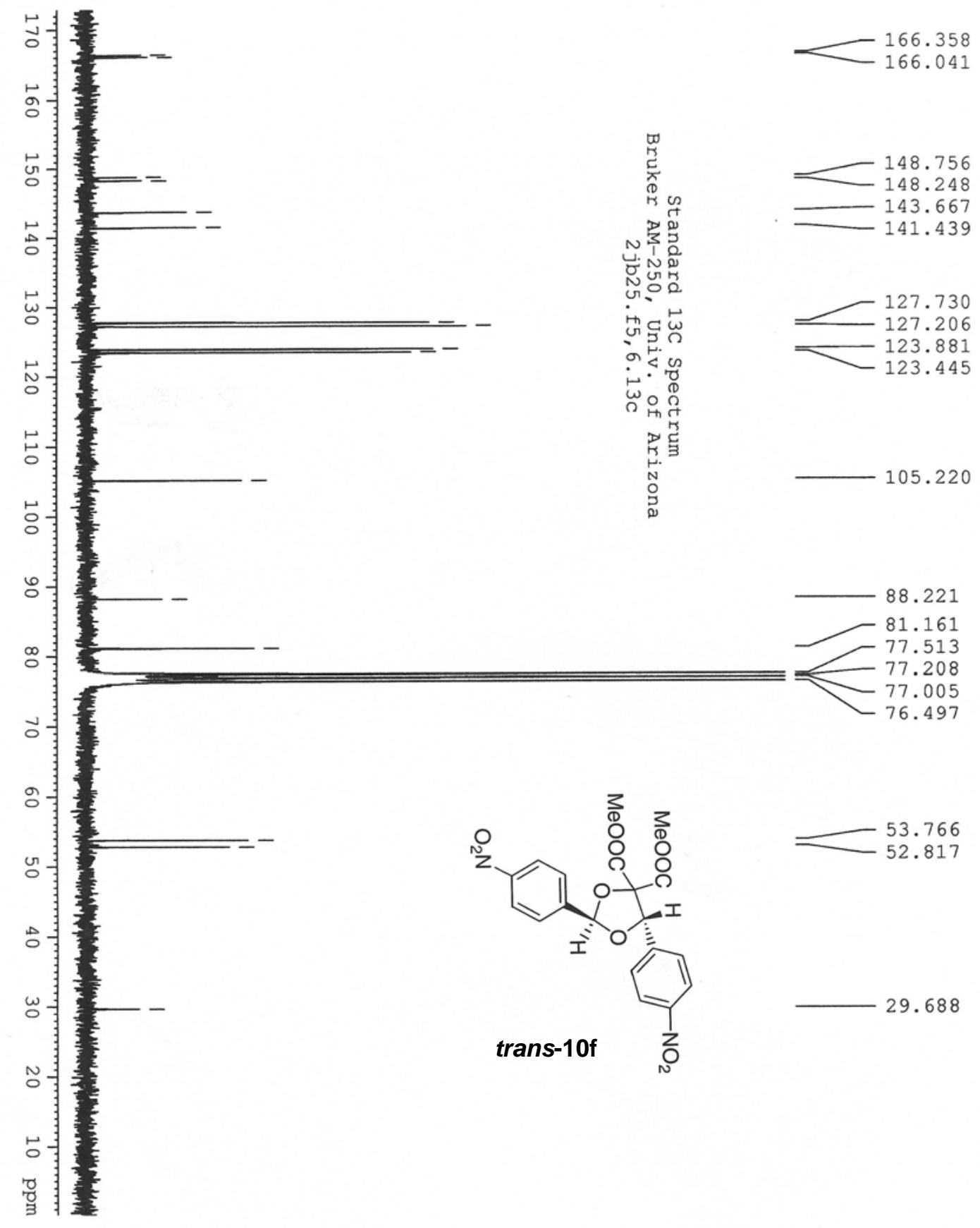




\section{M.P. Doyle et al. - Supporting Information}

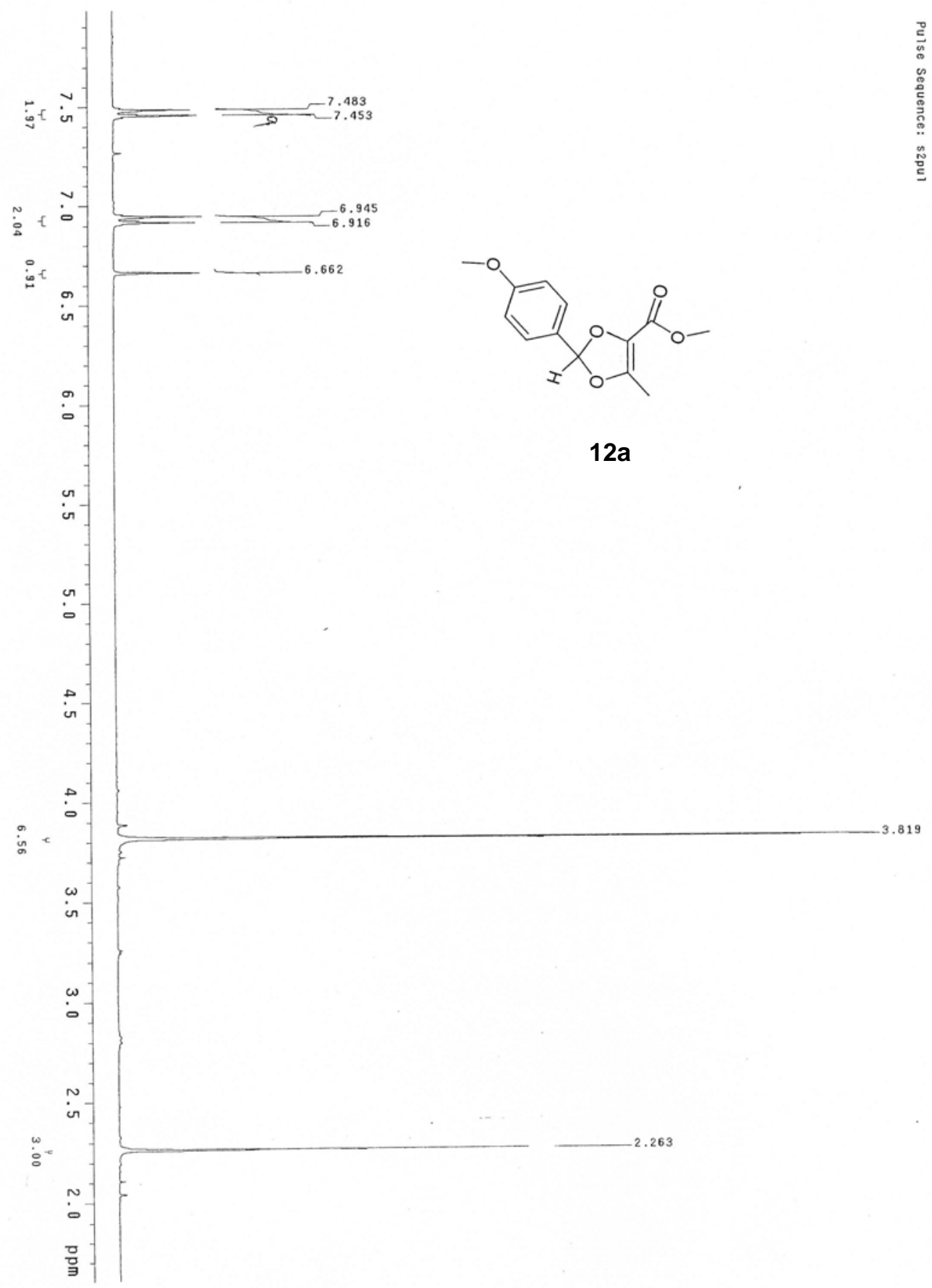




\section{M.P. Doyle et al. - Supporting Information}

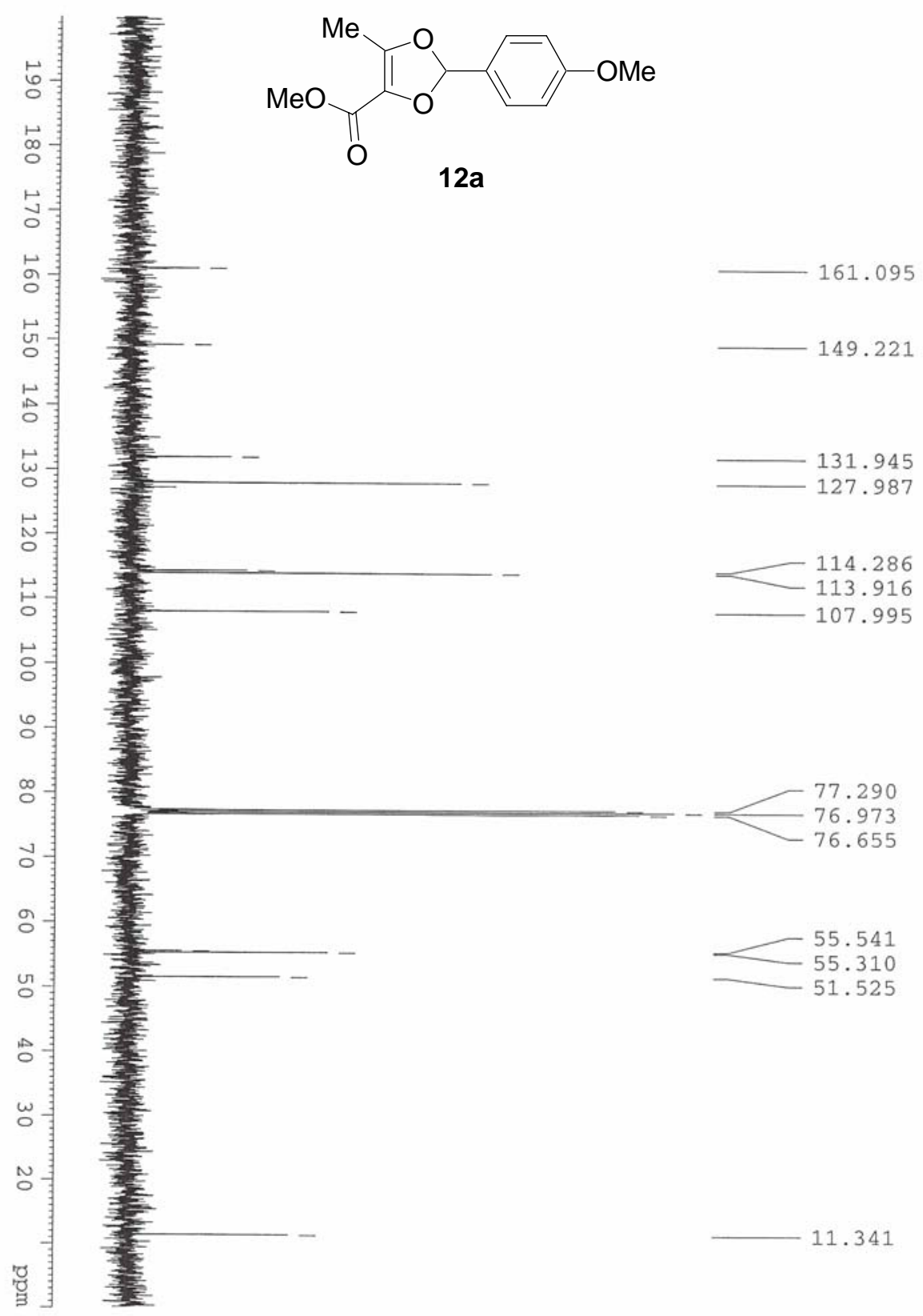




\section{M.P. Doyle et al. - Supporting Information}

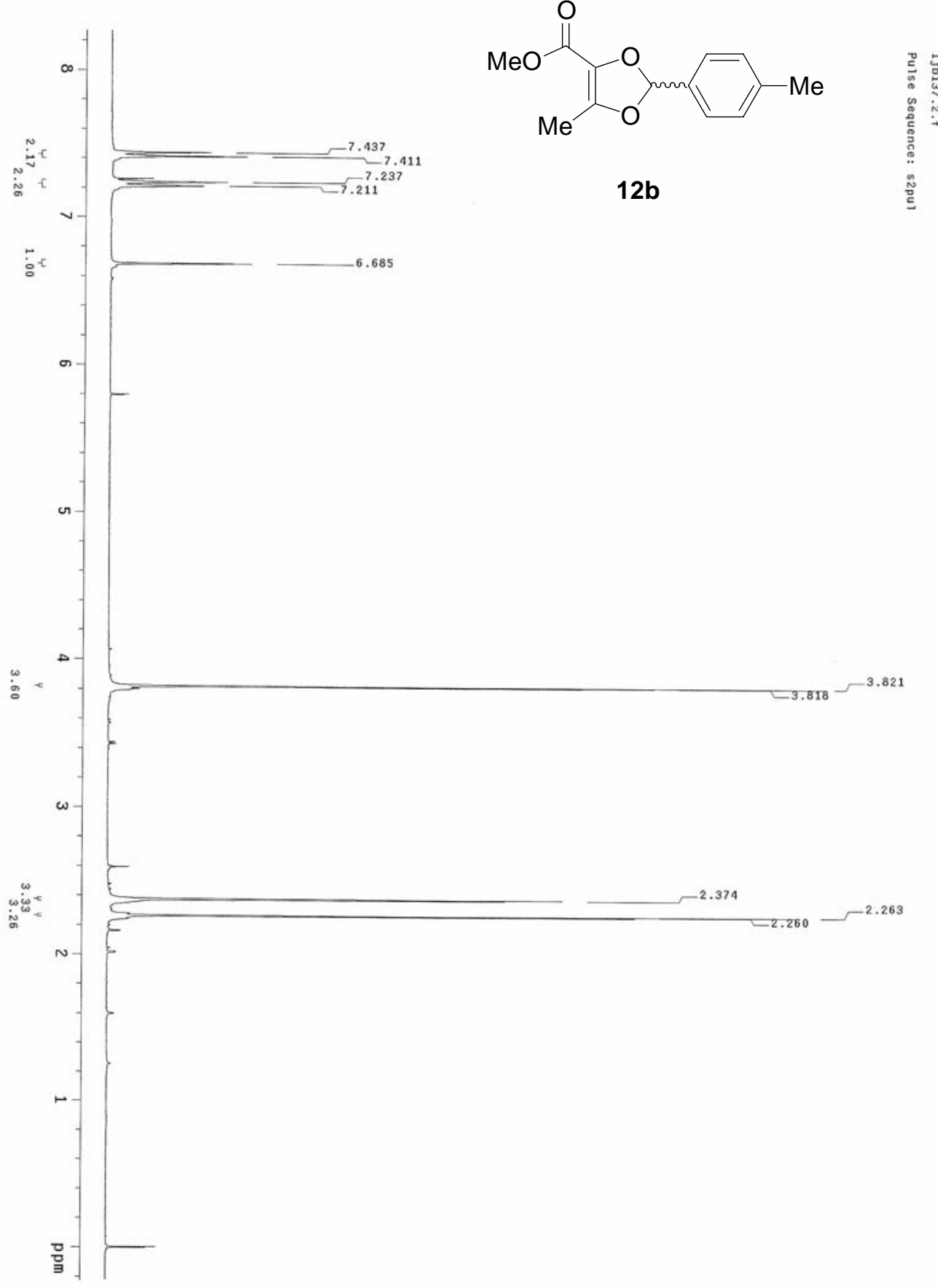


M.P. Doyle et al. - Supporting Information

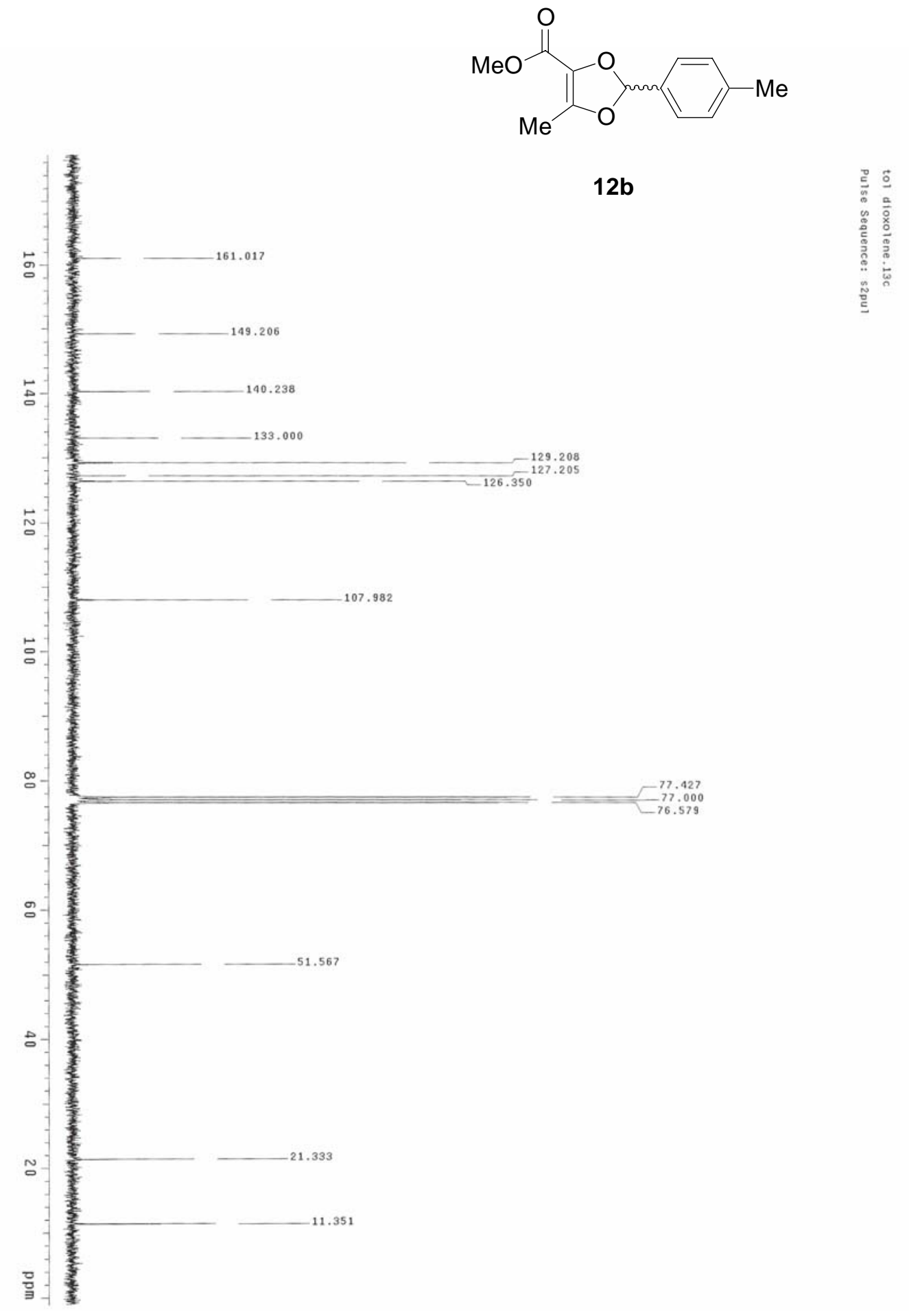




\section{M.P. Doyle et al. - Supporting Information}

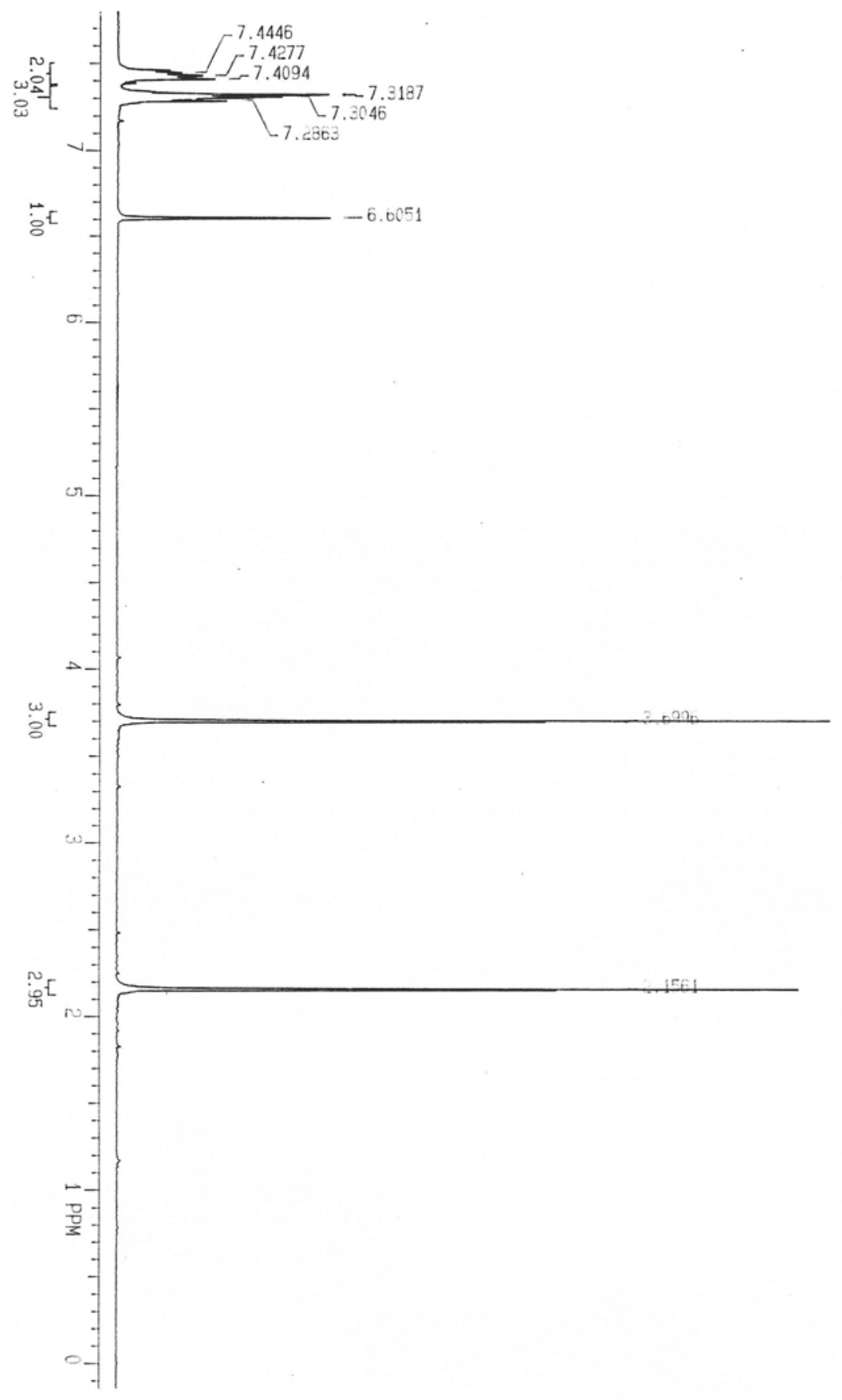

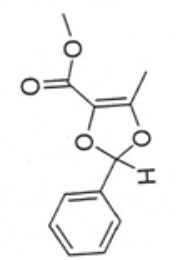

12c 


\section{M.P. Doyle et al. - Supporting Information}

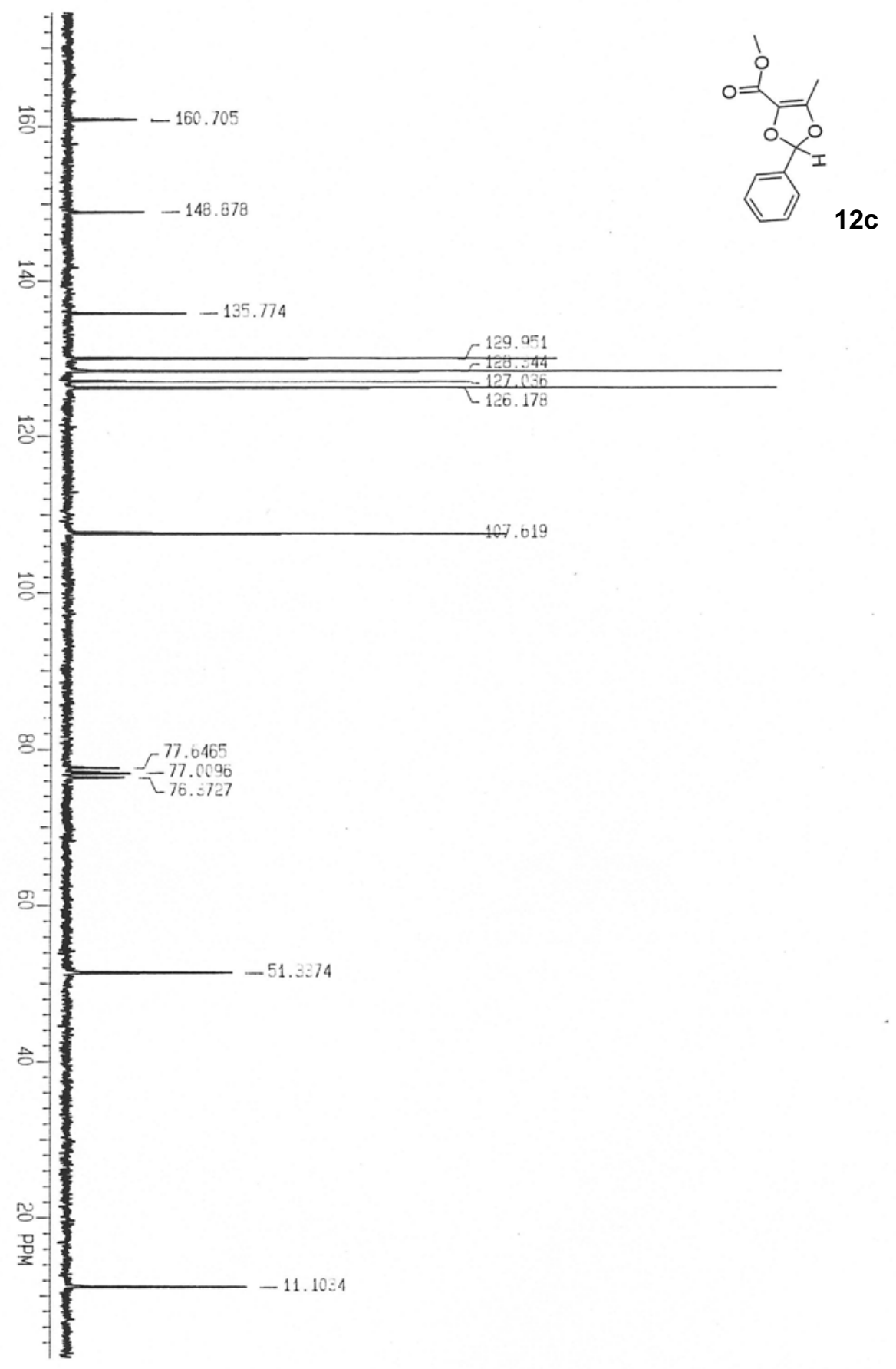




\section{M.P. Doyle et al. - Supporting Information}

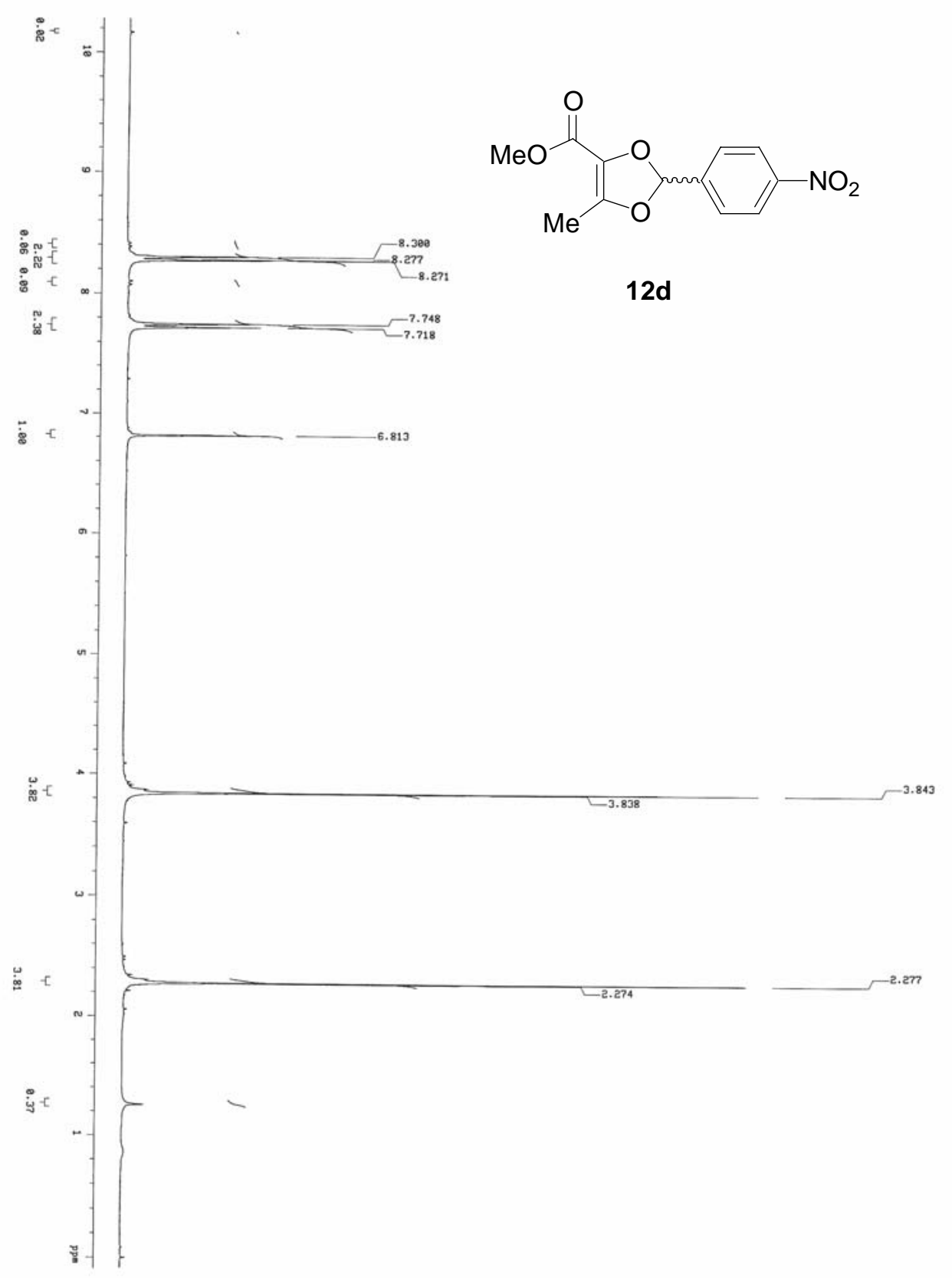


M.P. Doyle et al. - Supporting Information

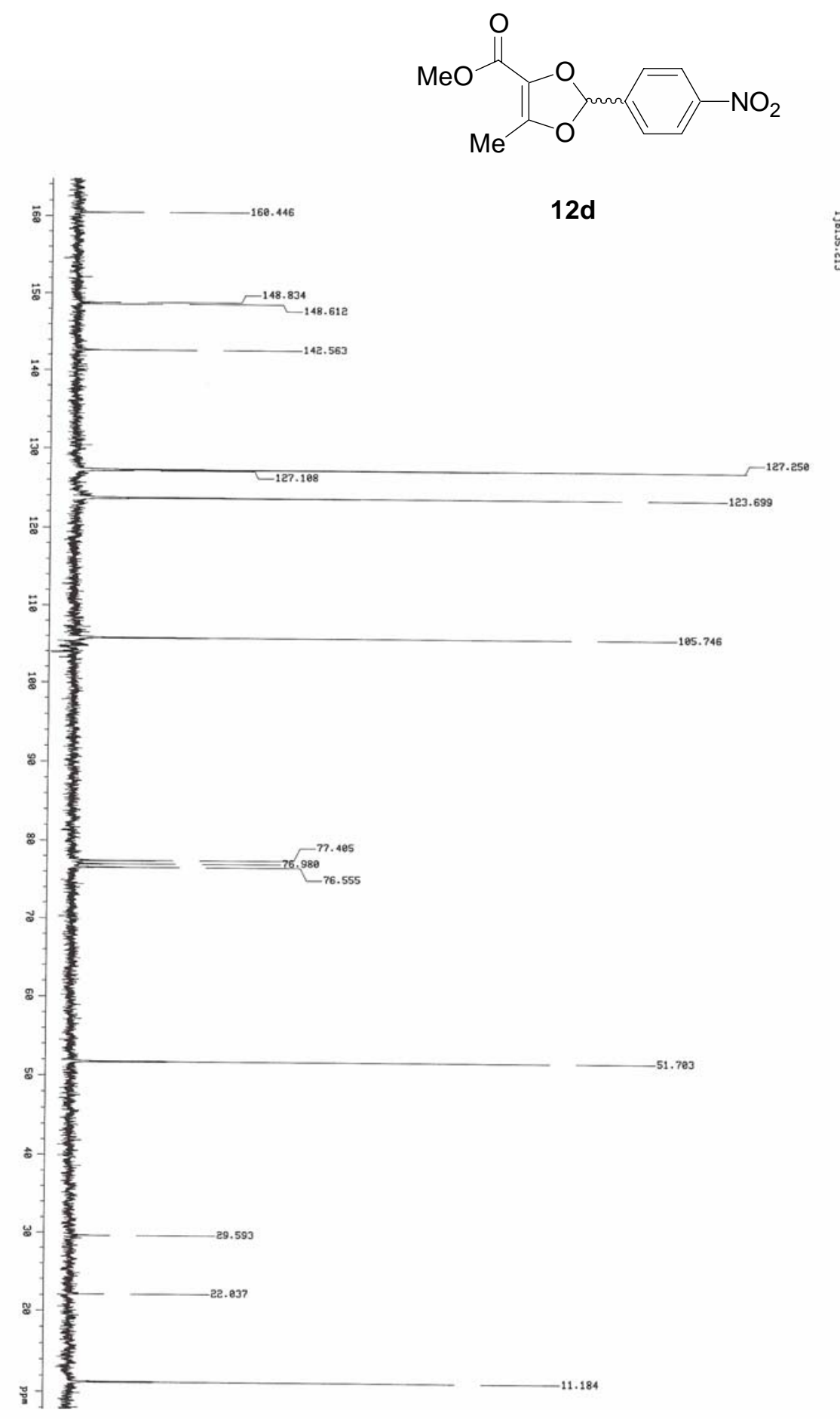




\section{M.P. Doyle et al. - Supporting Information}

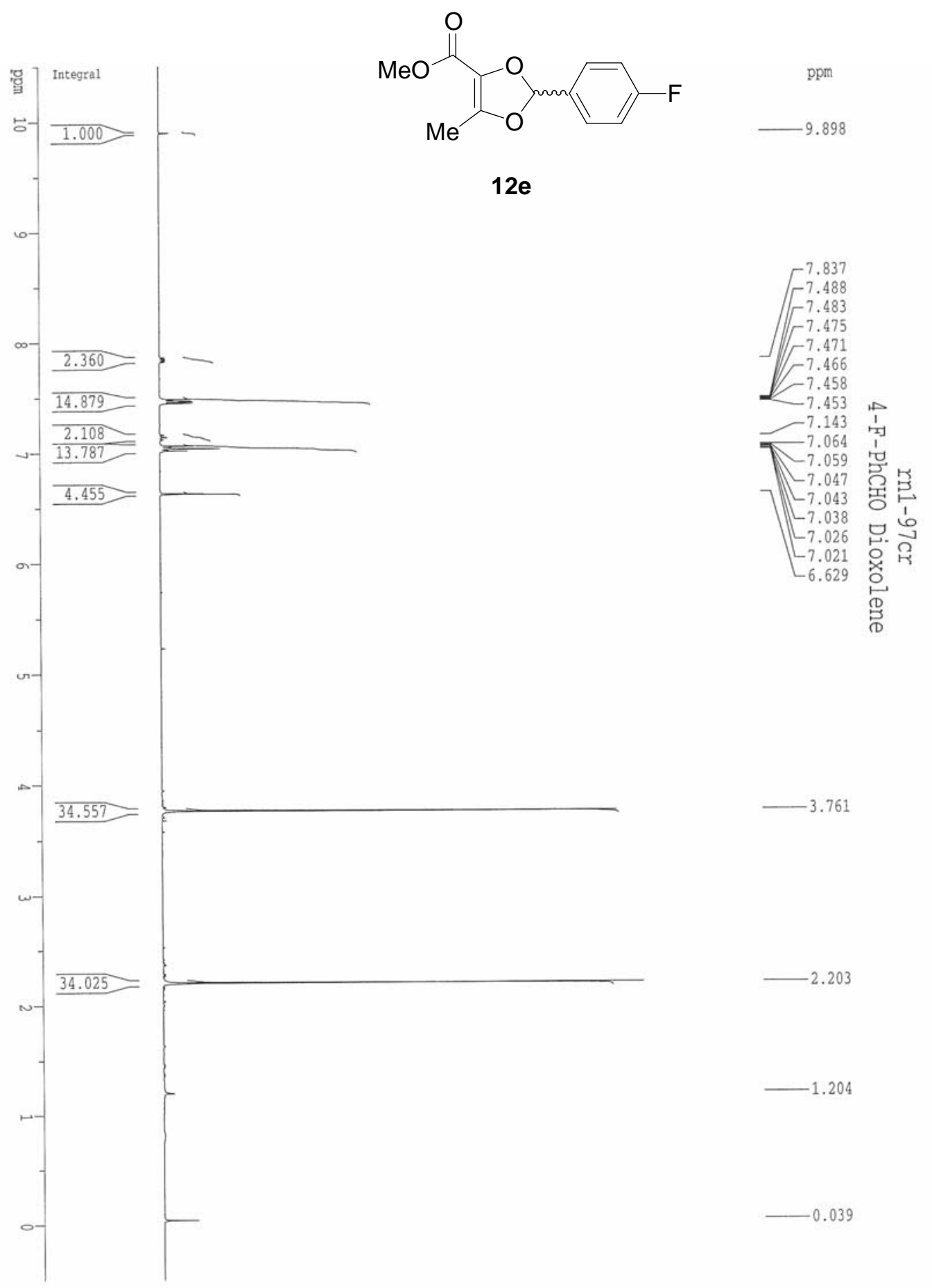




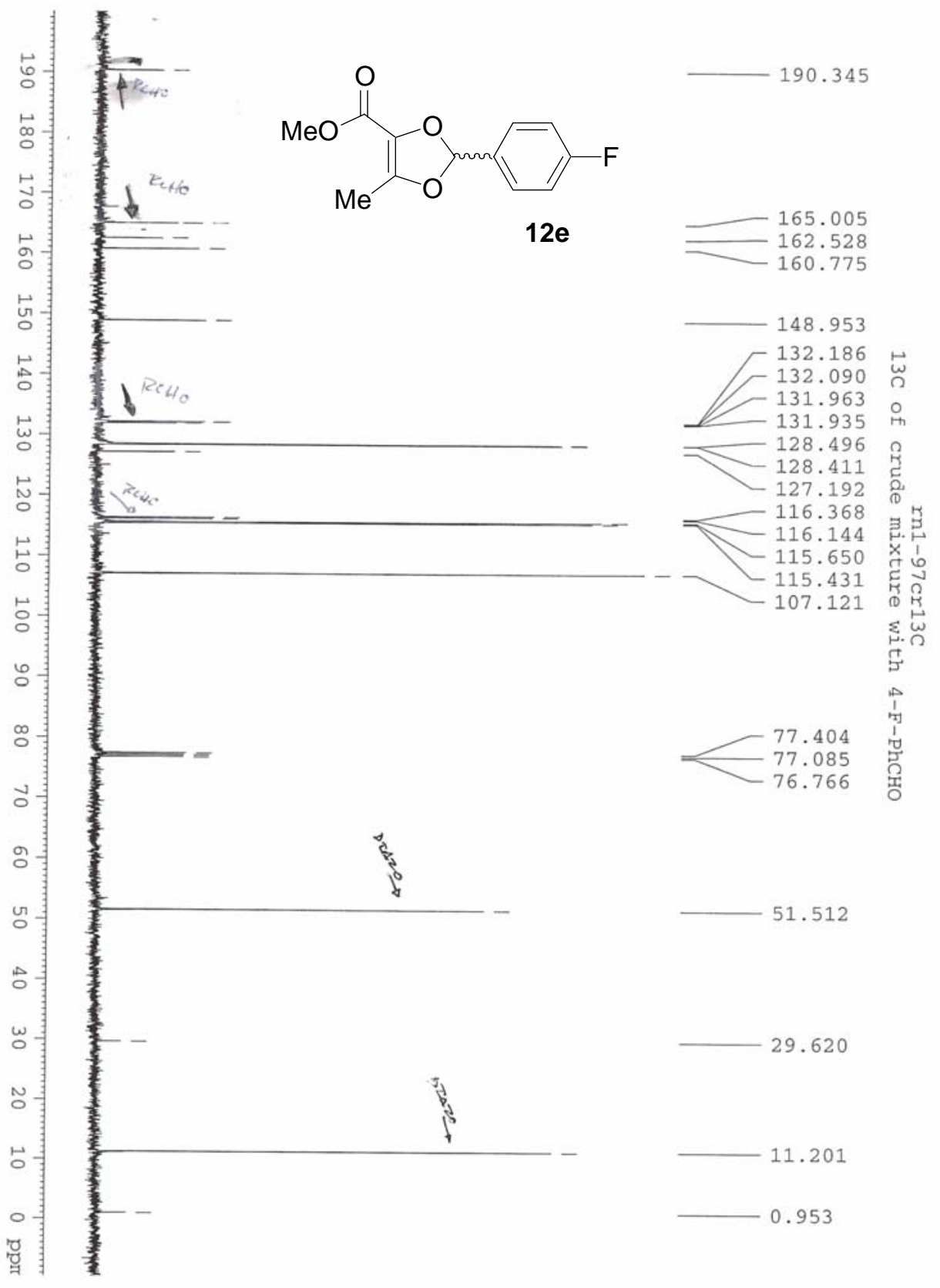




\section{M.P. Doyle et al. - Supporting Information}

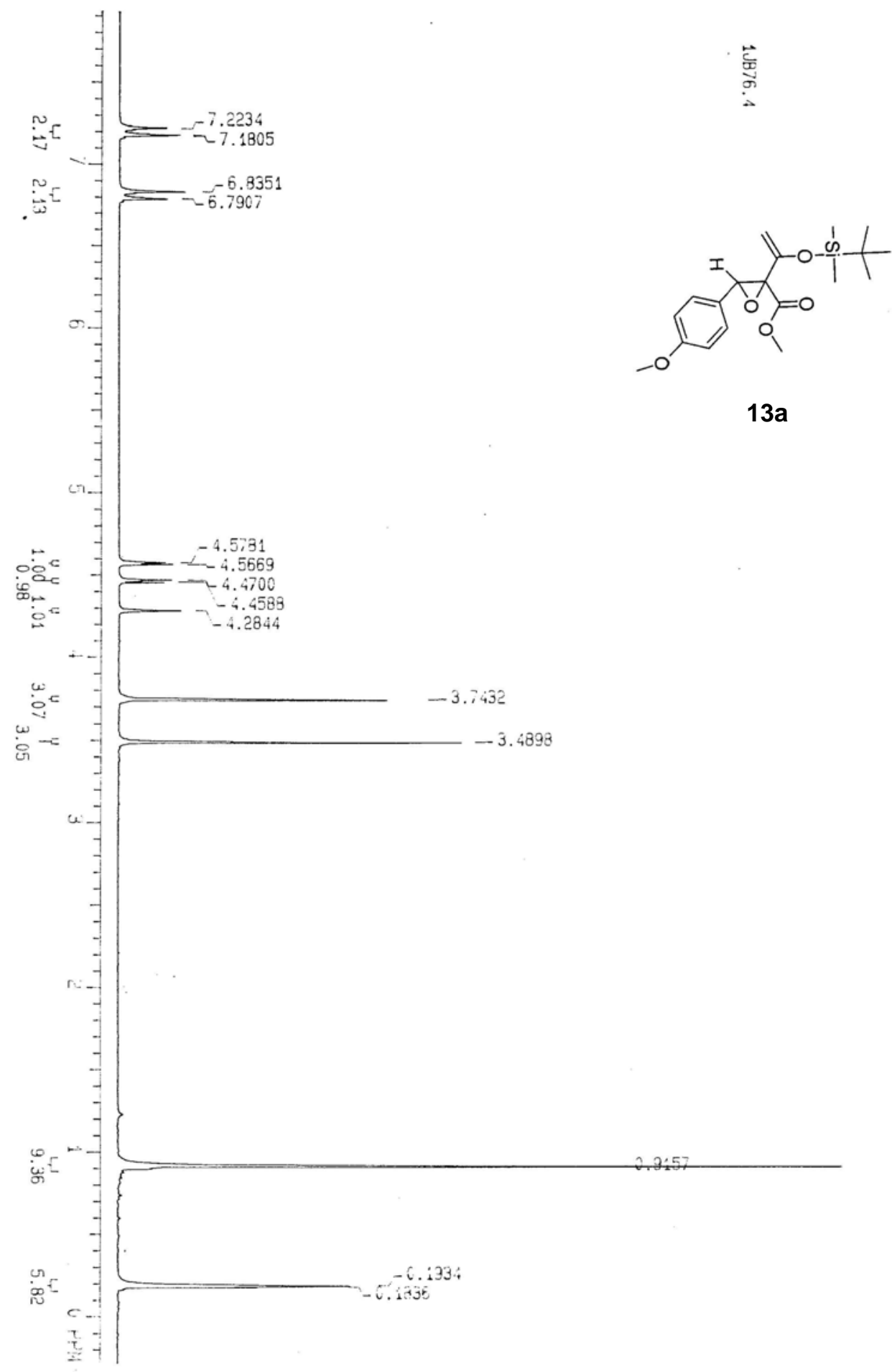




\section{M.P. Doyle et al. - Supporting Information}

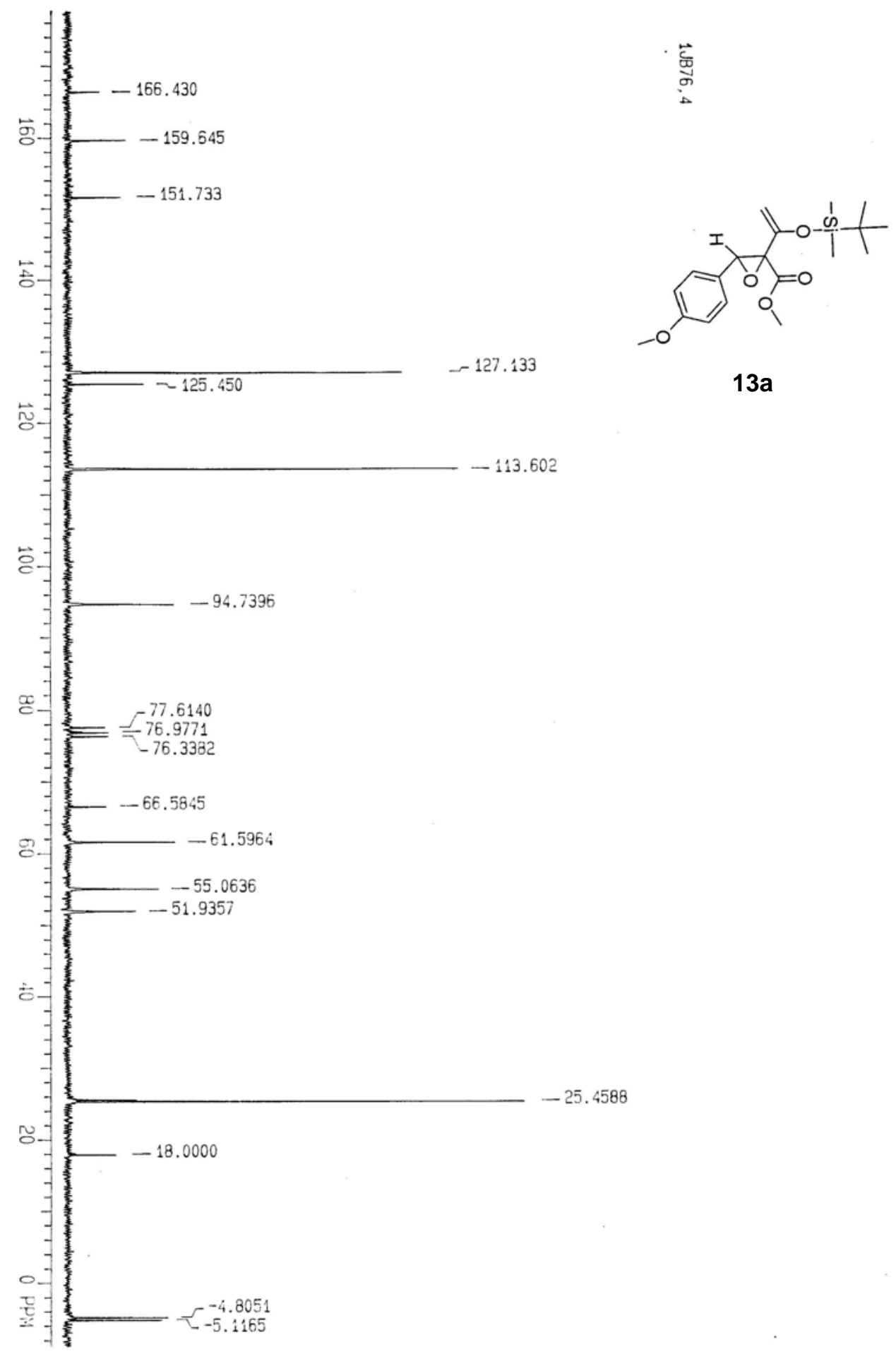




\section{M.P. Doyle et al. - Supporting Information}

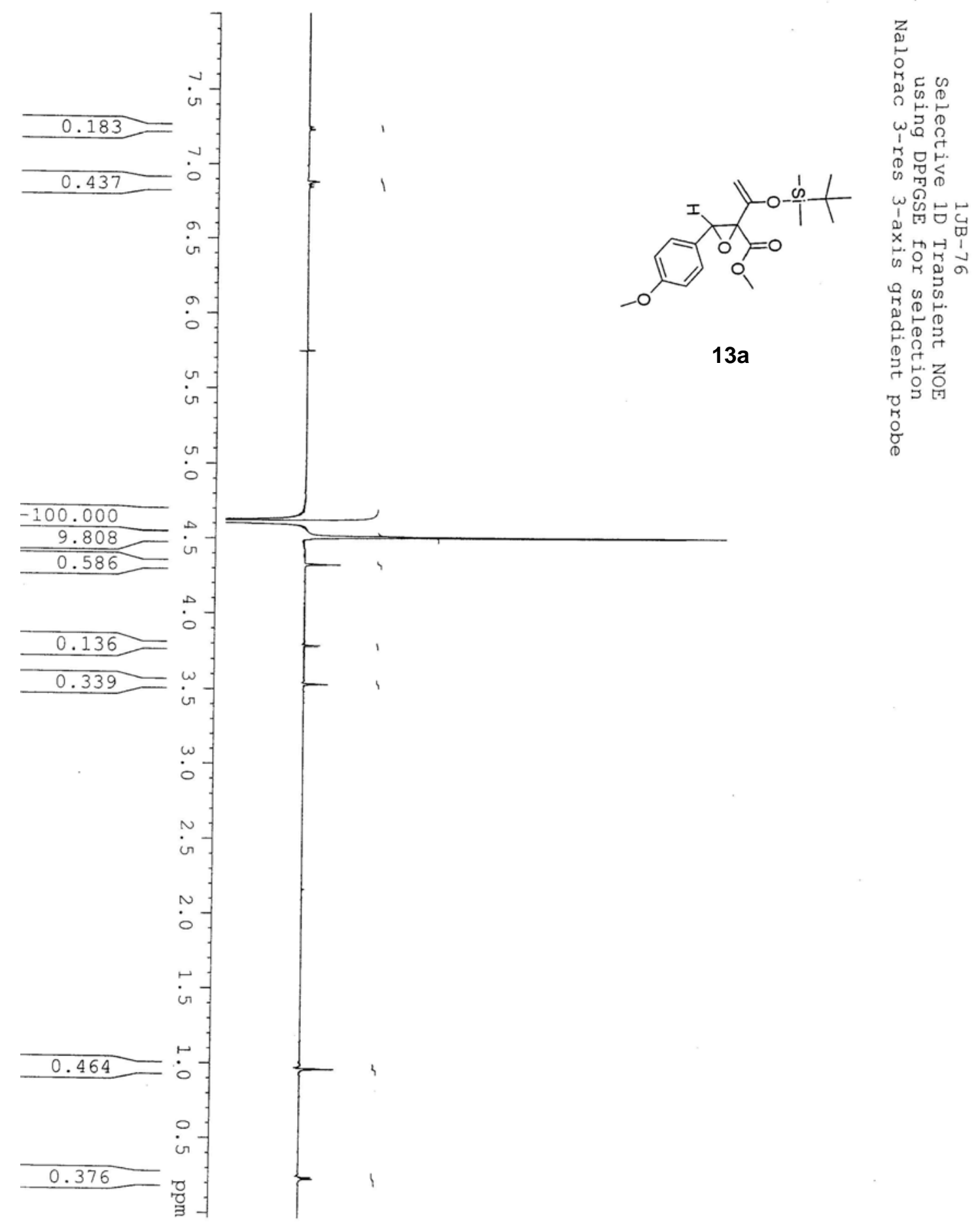




\section{M.P. Doyle et al. - Supporting Information}

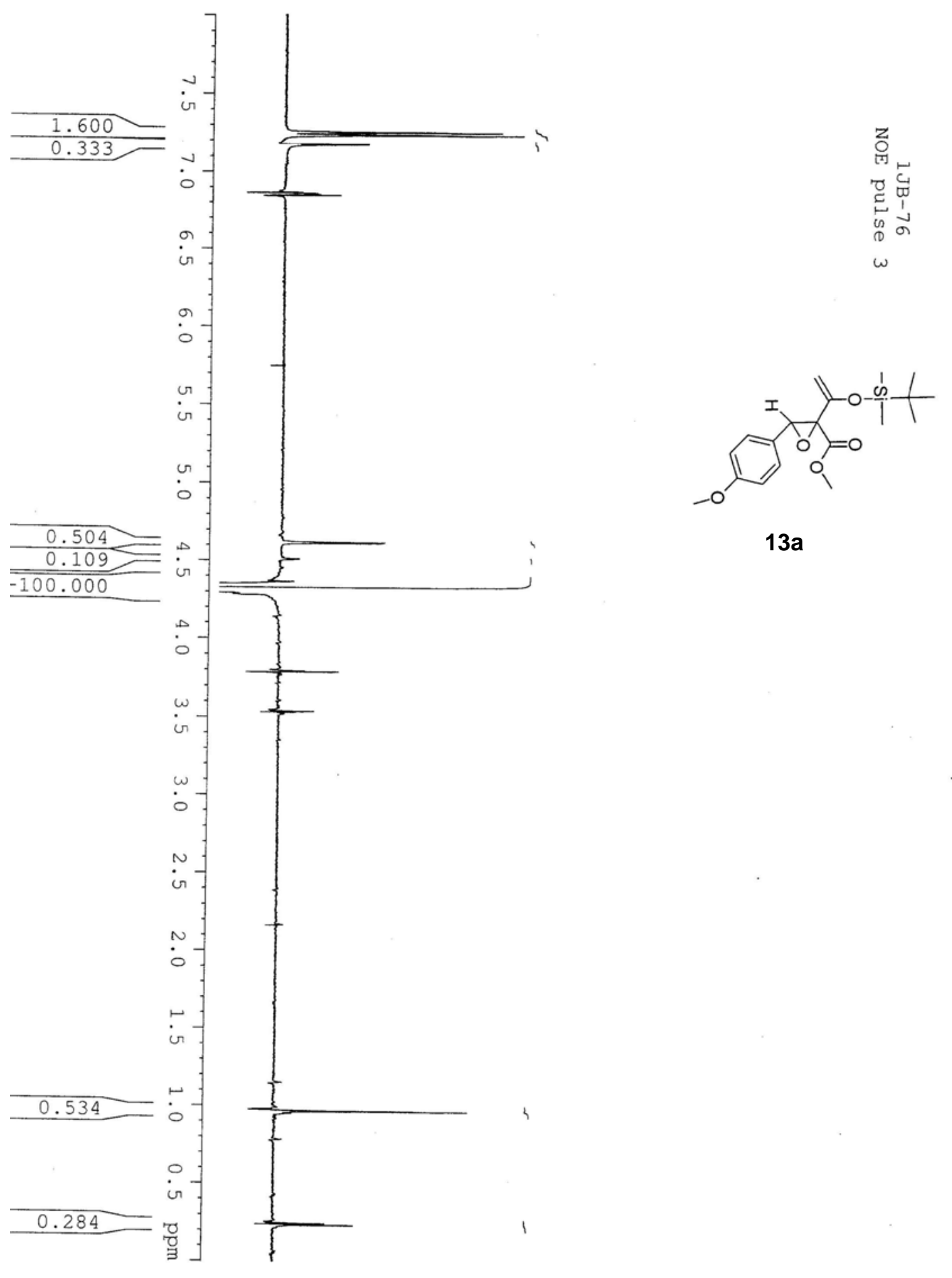




\section{M.P. Doyle et al. - Supporting Information}

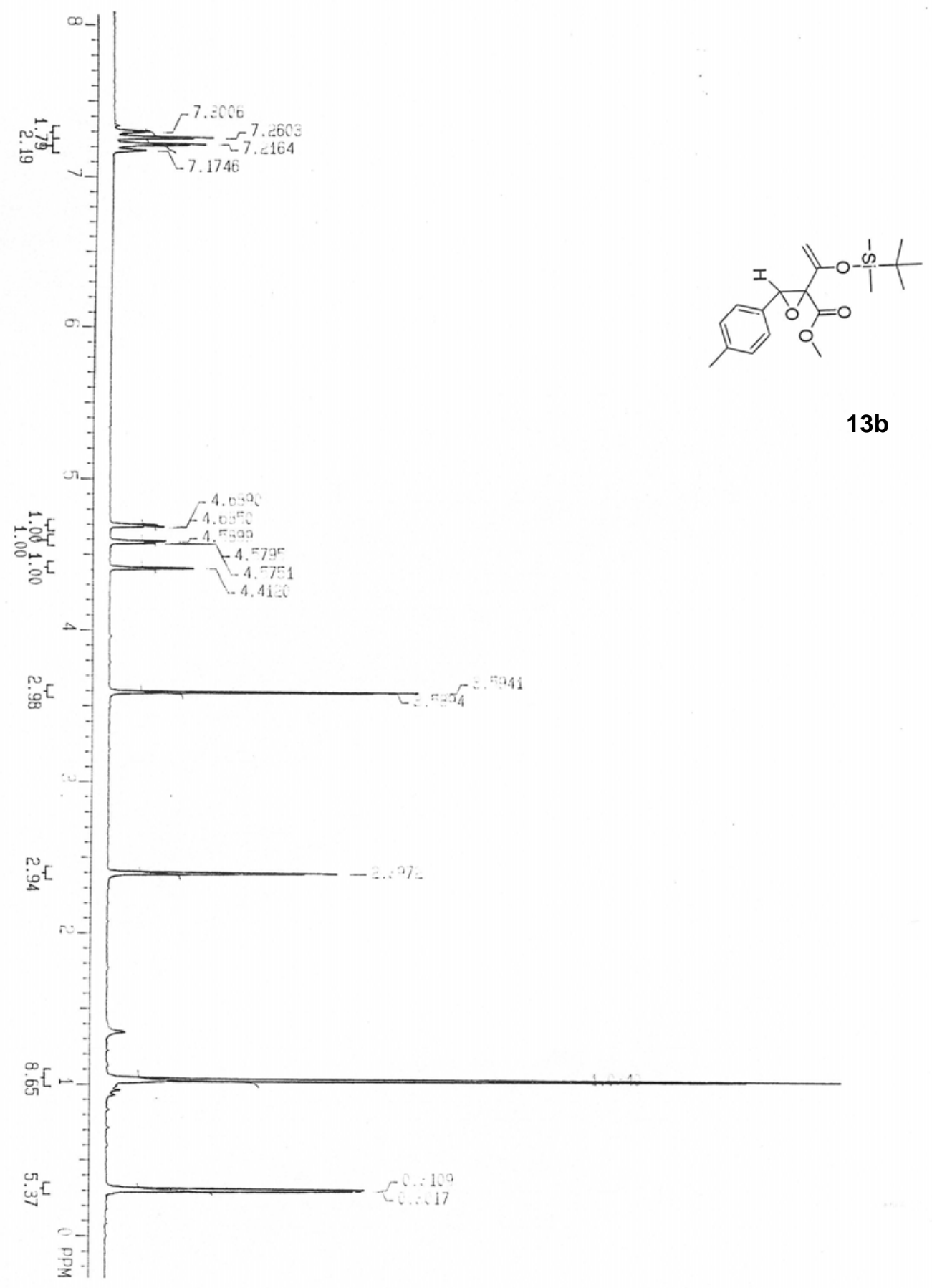




\section{M.P. Doyle et al. - Supporting Information}

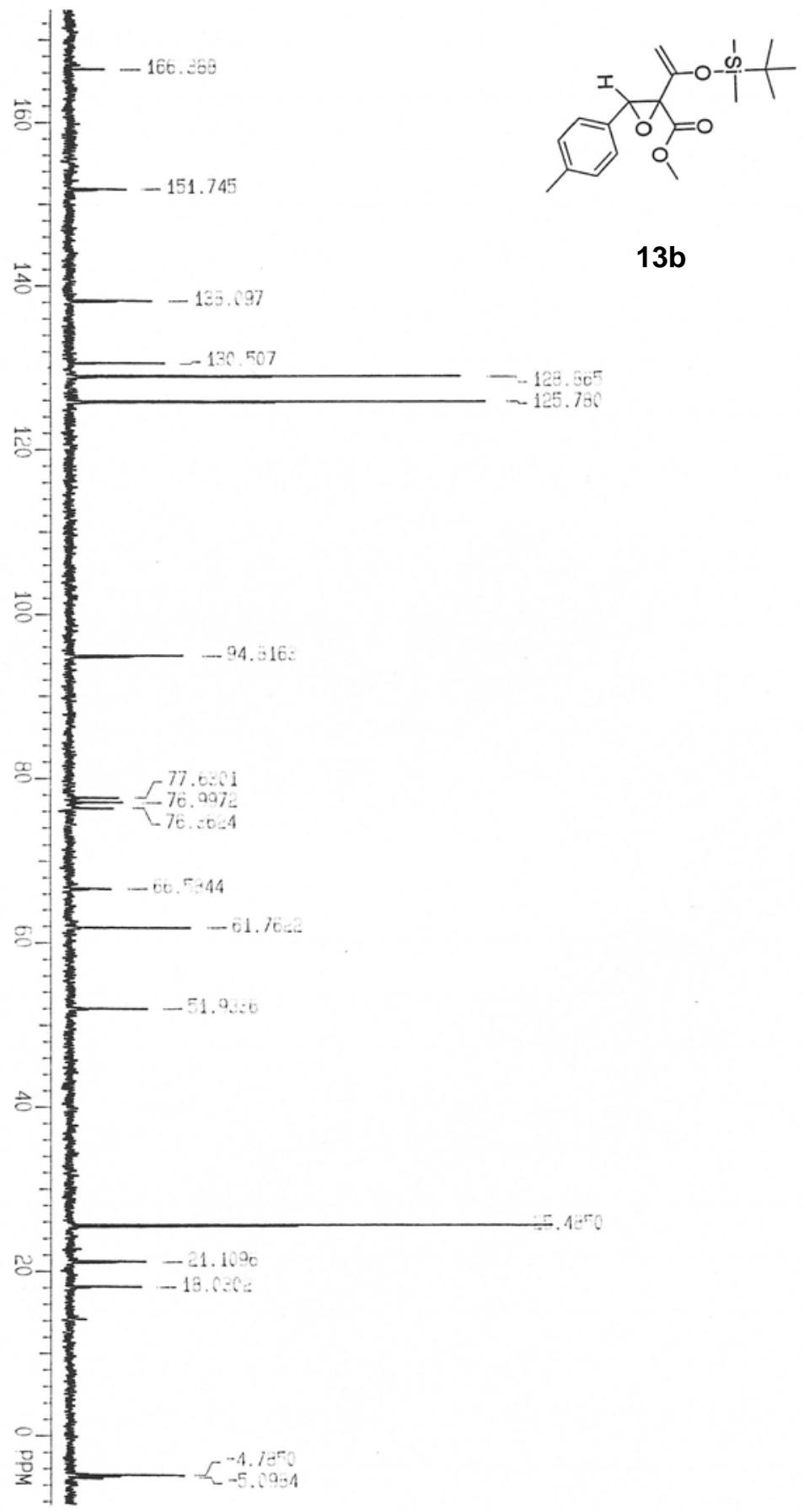




\section{M.P. Doyle et al. - Supporting Information}

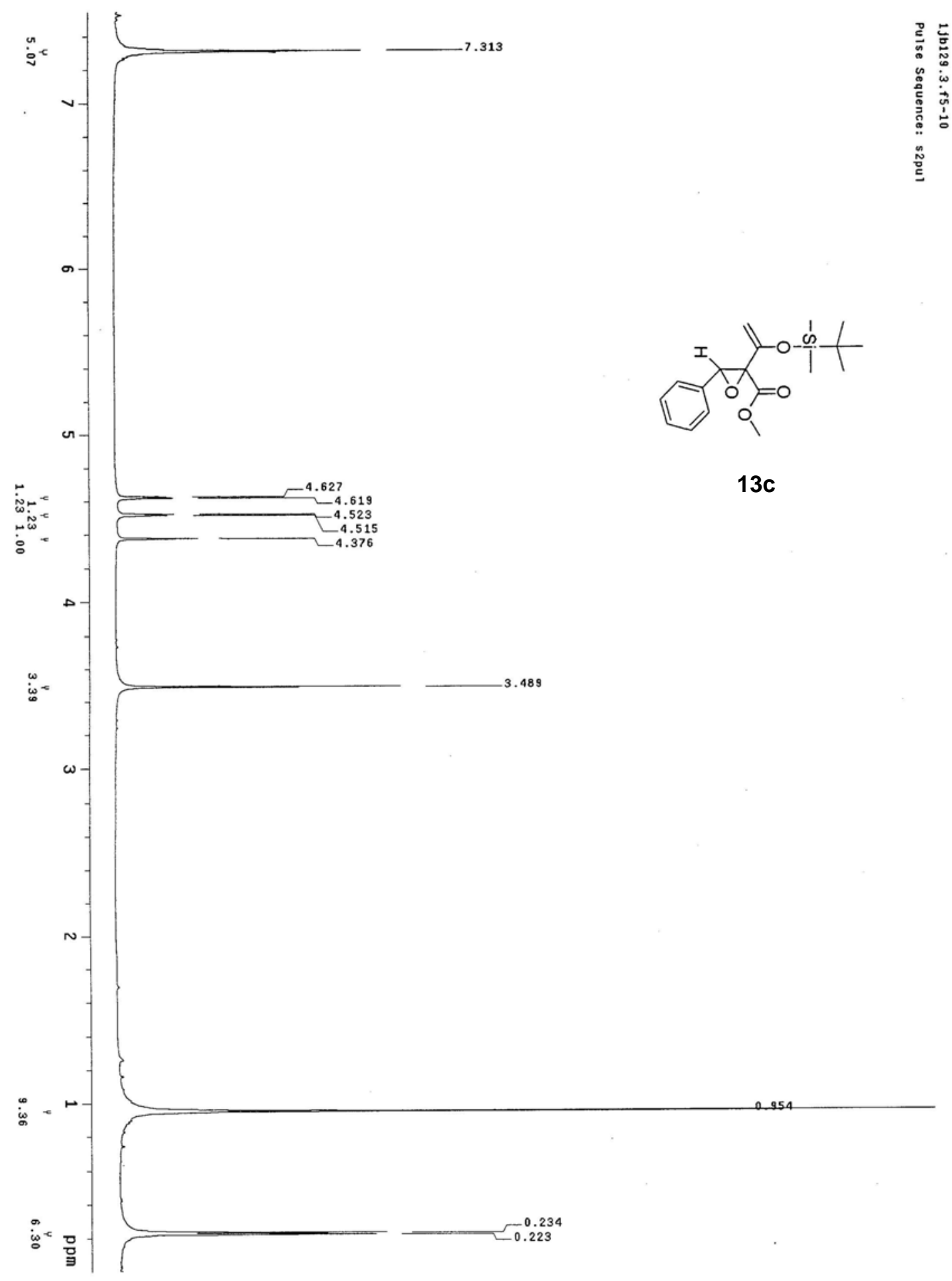




\section{M.P. Doyle et al. - Supporting Information}

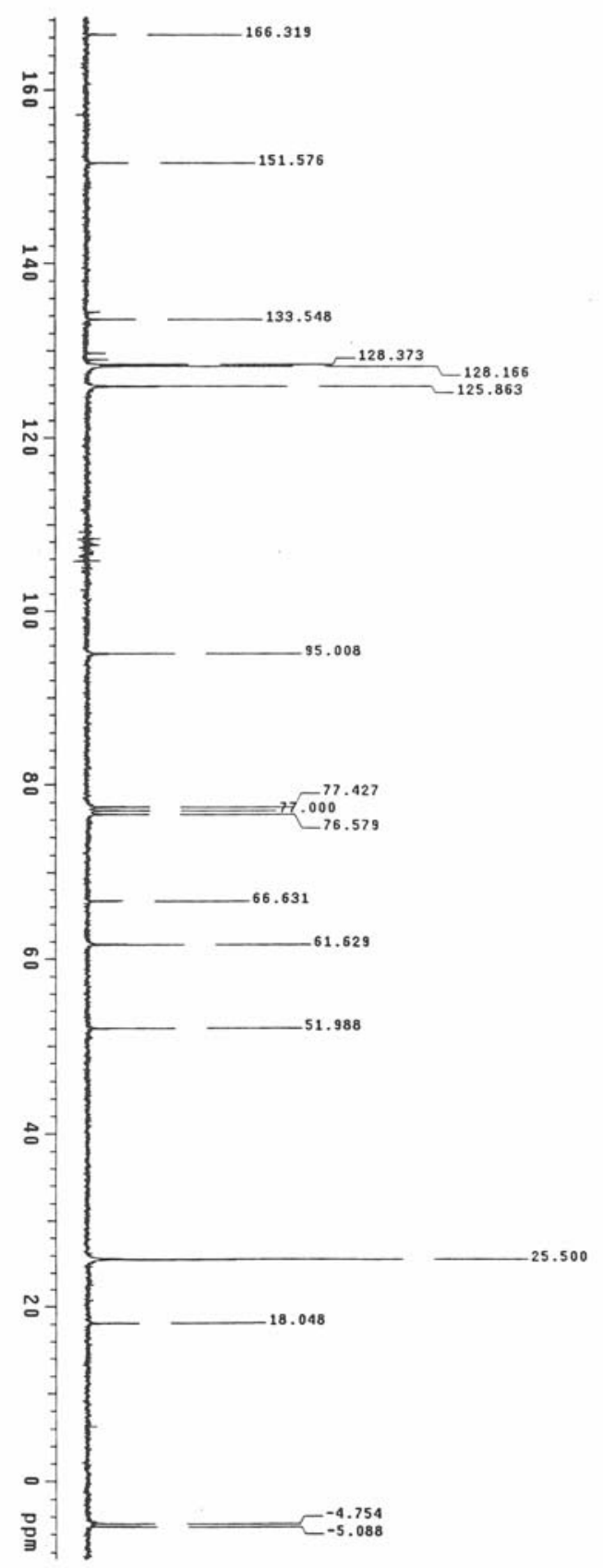

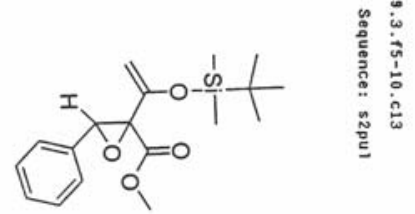

$13 c$ 
M.P. Doyle et al. - Supporting Information

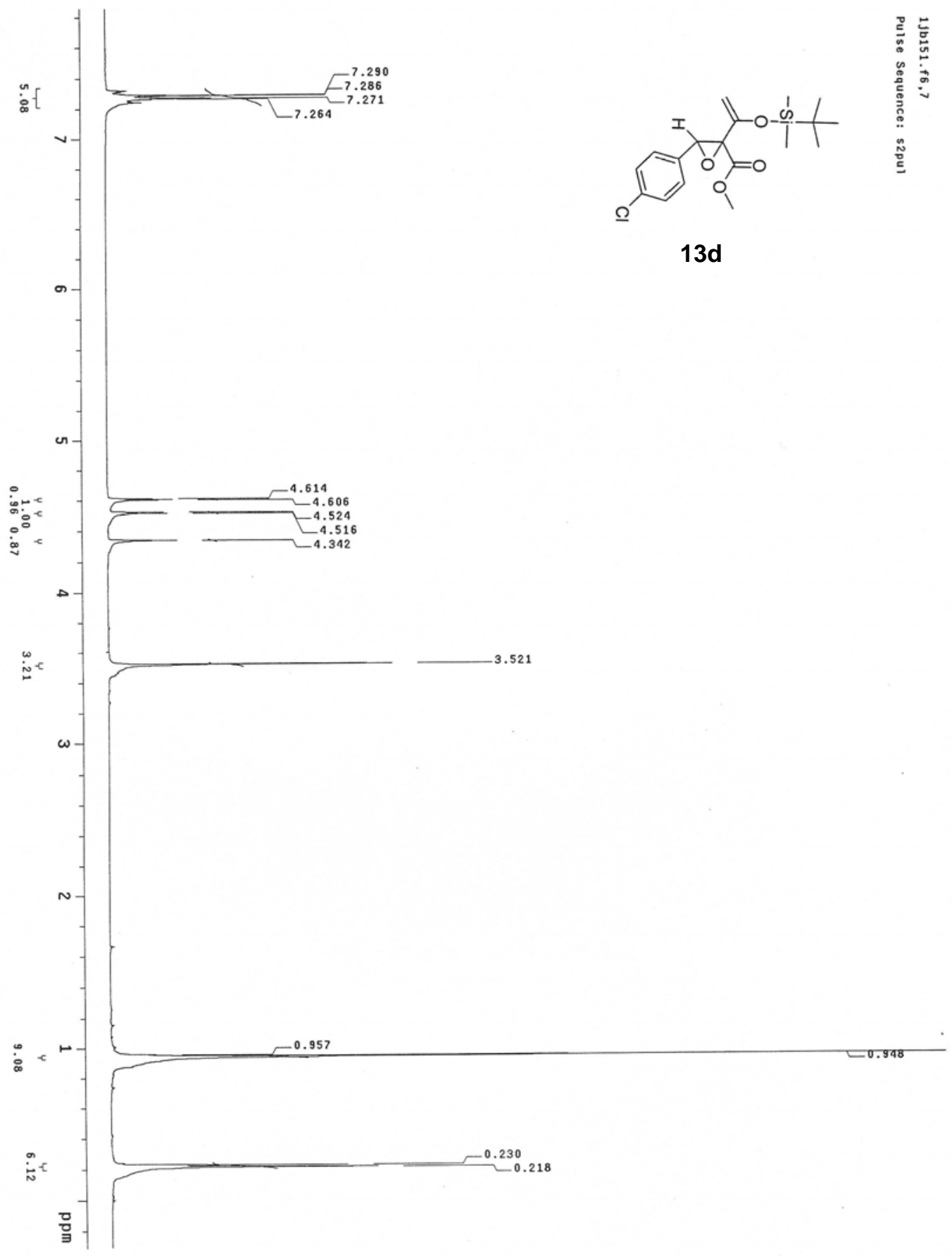




\section{M.P. Doyle et al. - Supporting Information}

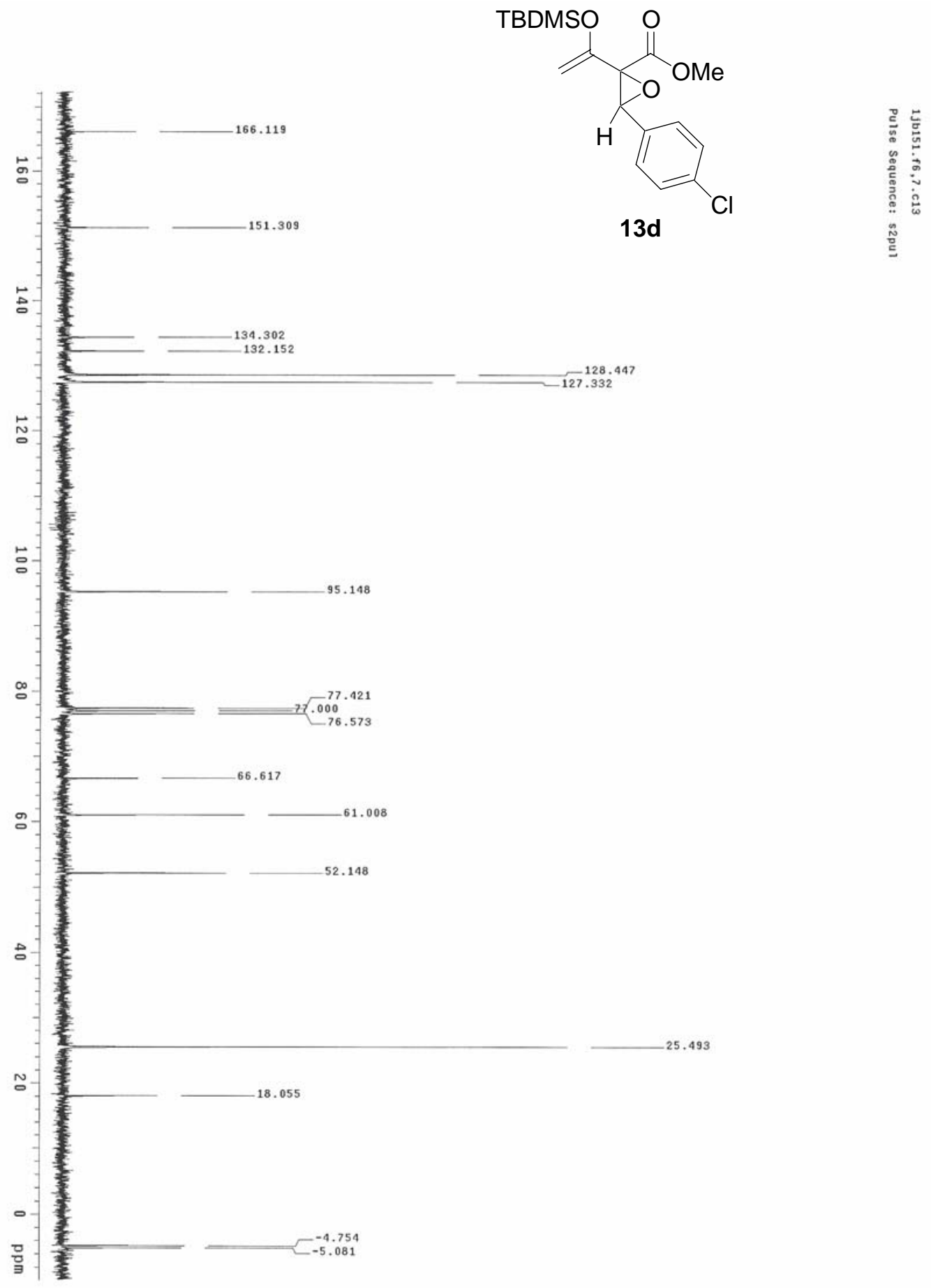




\section{M.P. Doyle et al. - Supporting Information}

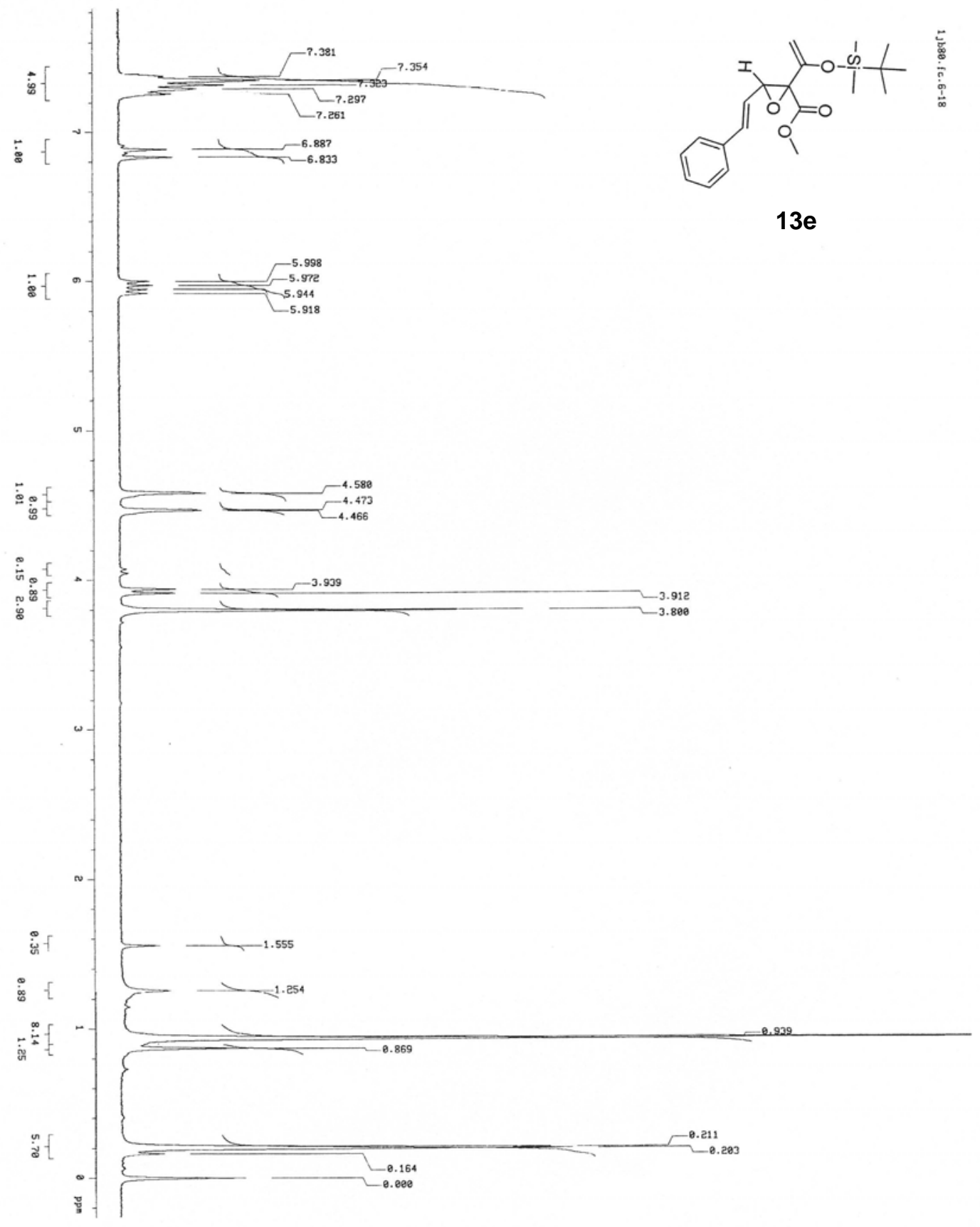




\section{M.P. Doyle et al. - Supporting Information}

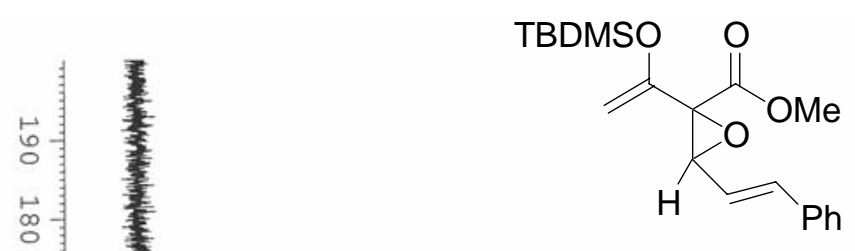

$13 e$

167.336
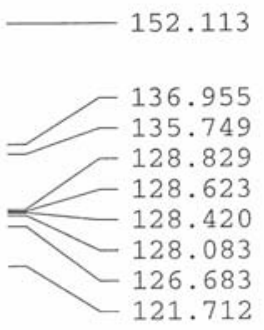

$\therefore$
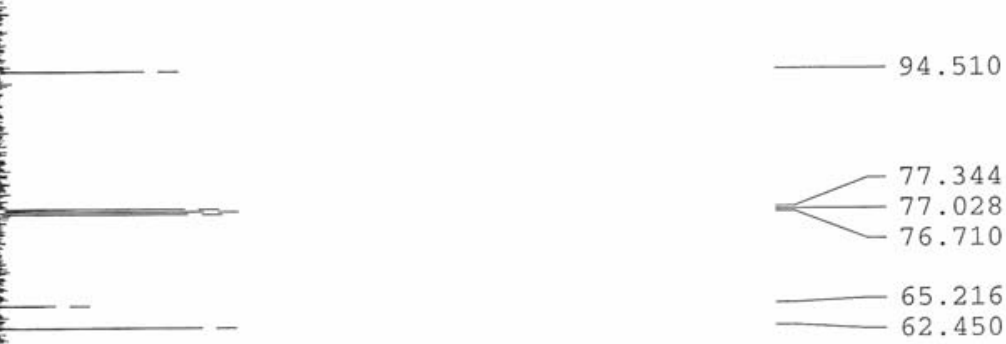

52.477

w

N

당

$\circ$ 年

뭉
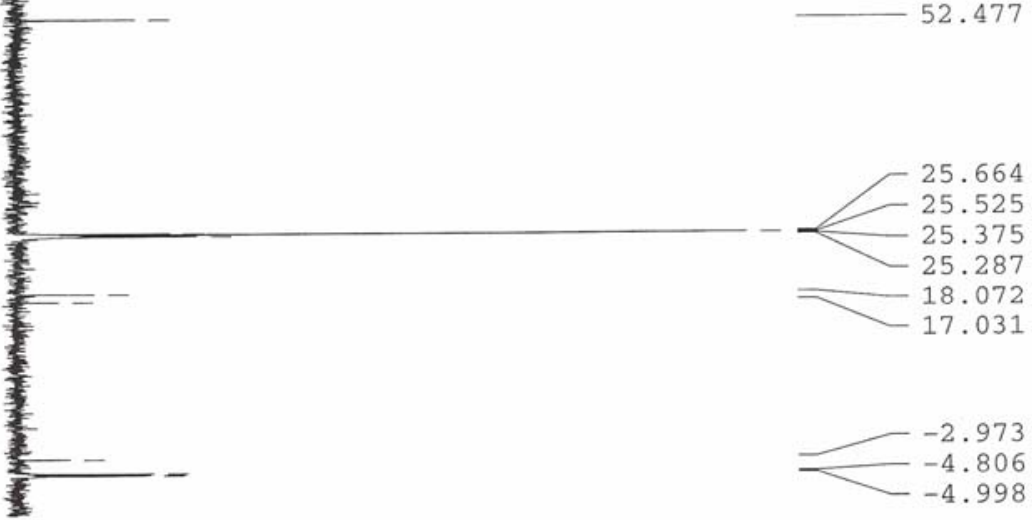


\section{M.P. Doyle et al. - Supporting Information}

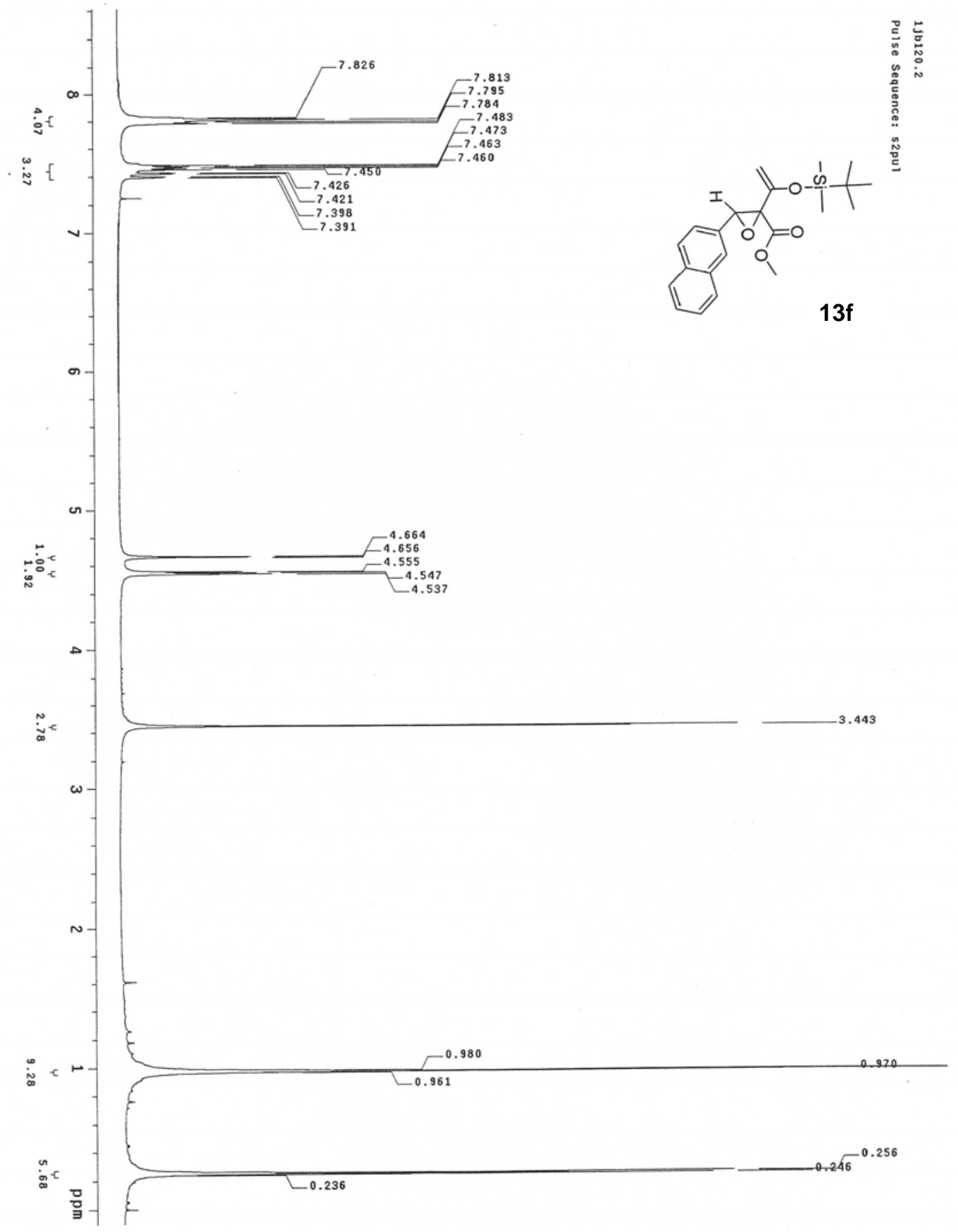


M.P. Doyle et al. - Supporting Information

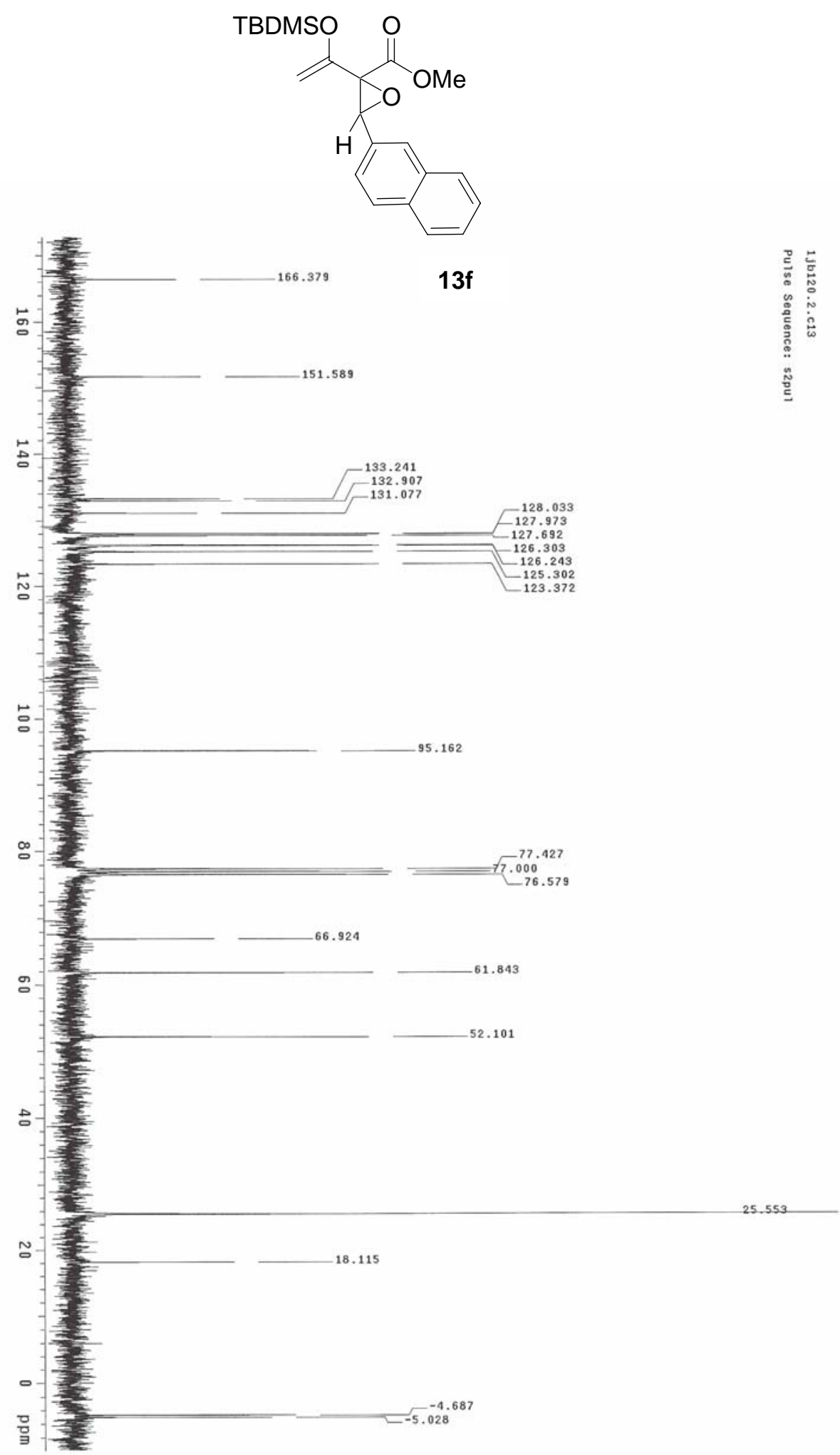




\section{M.P. Doyle et al. - Supporting Information}

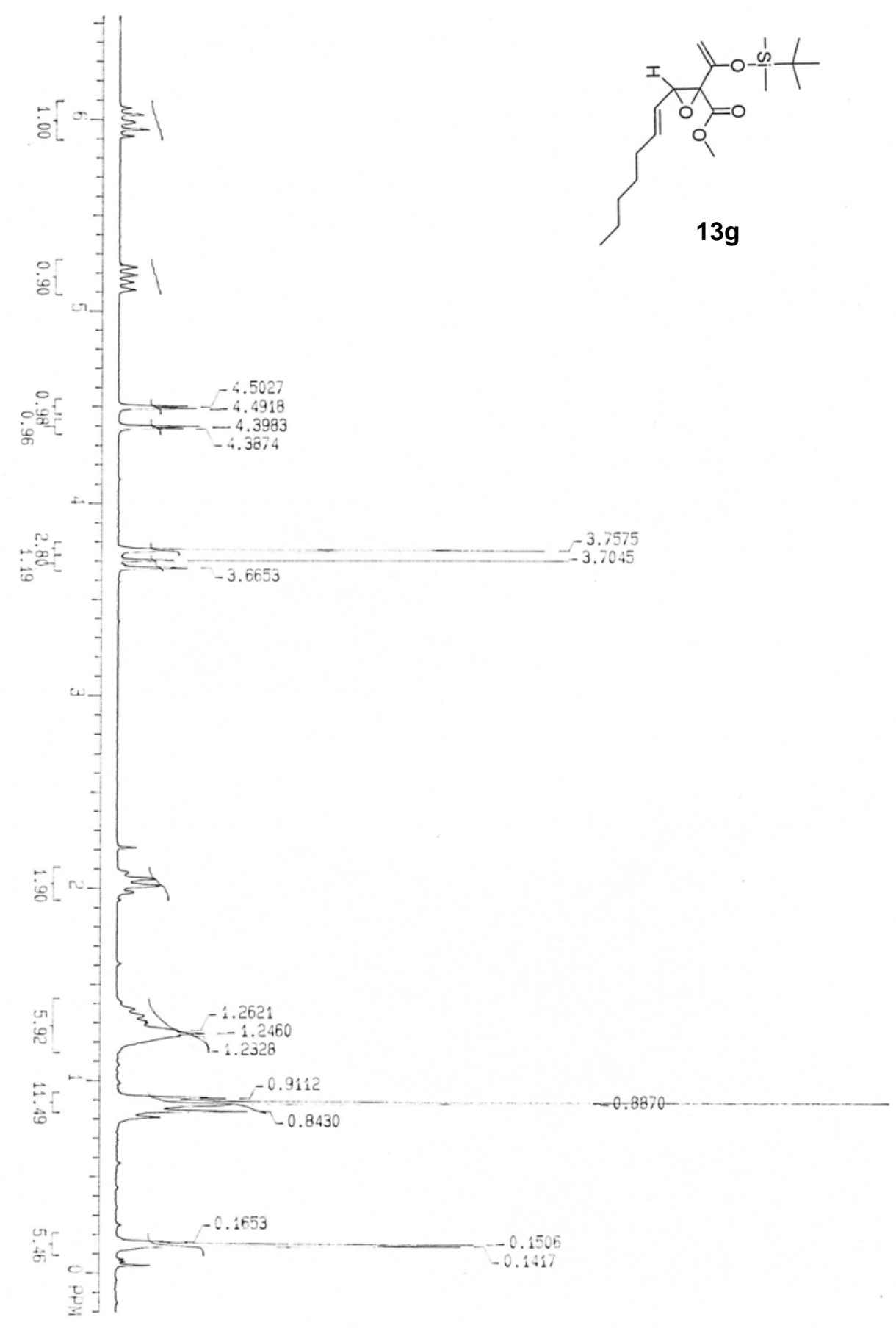




\section{M.P. Doyle et al. - Supporting Information}

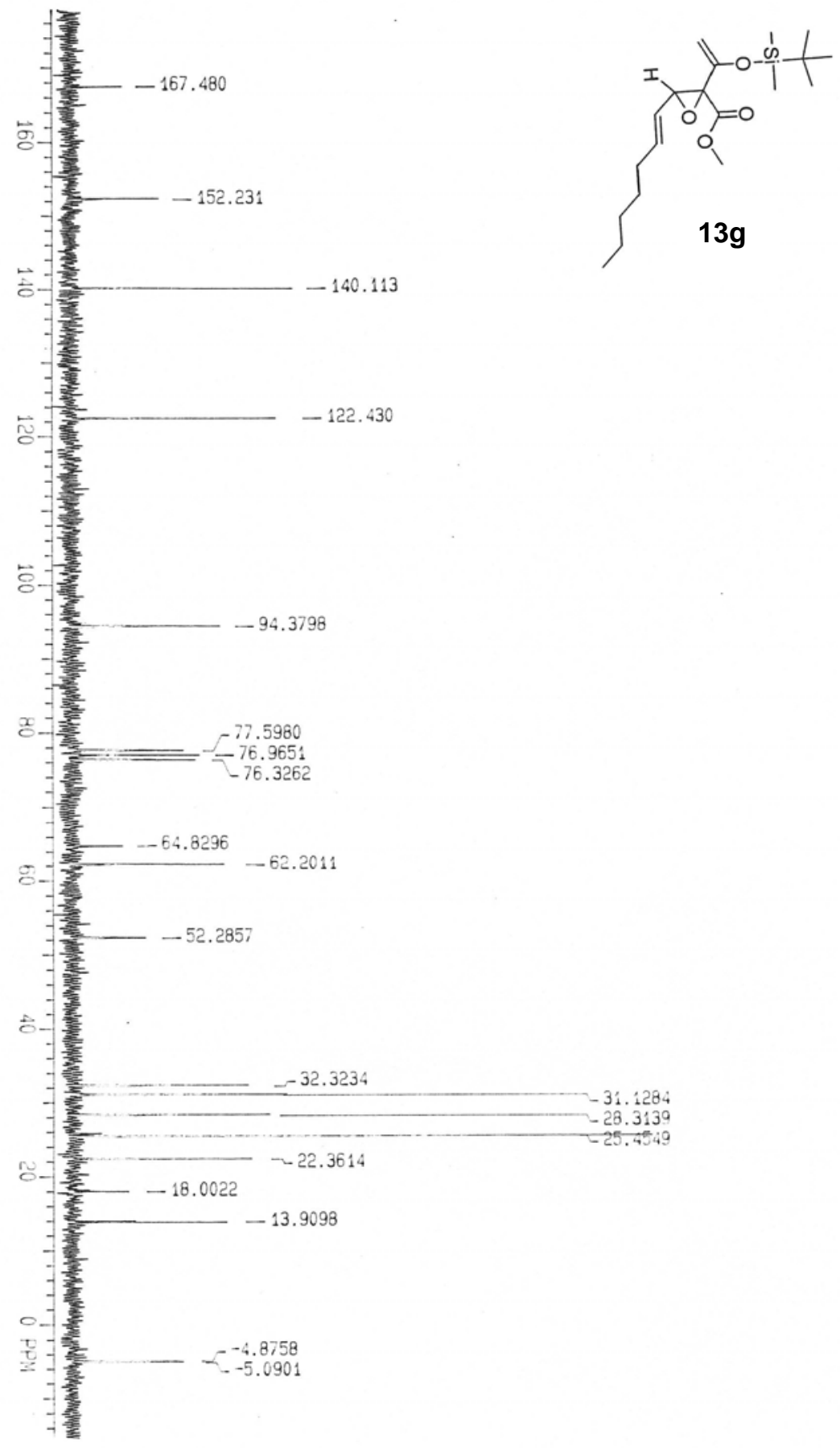




\section{M.P. Doyle et al. - Supporting Information}

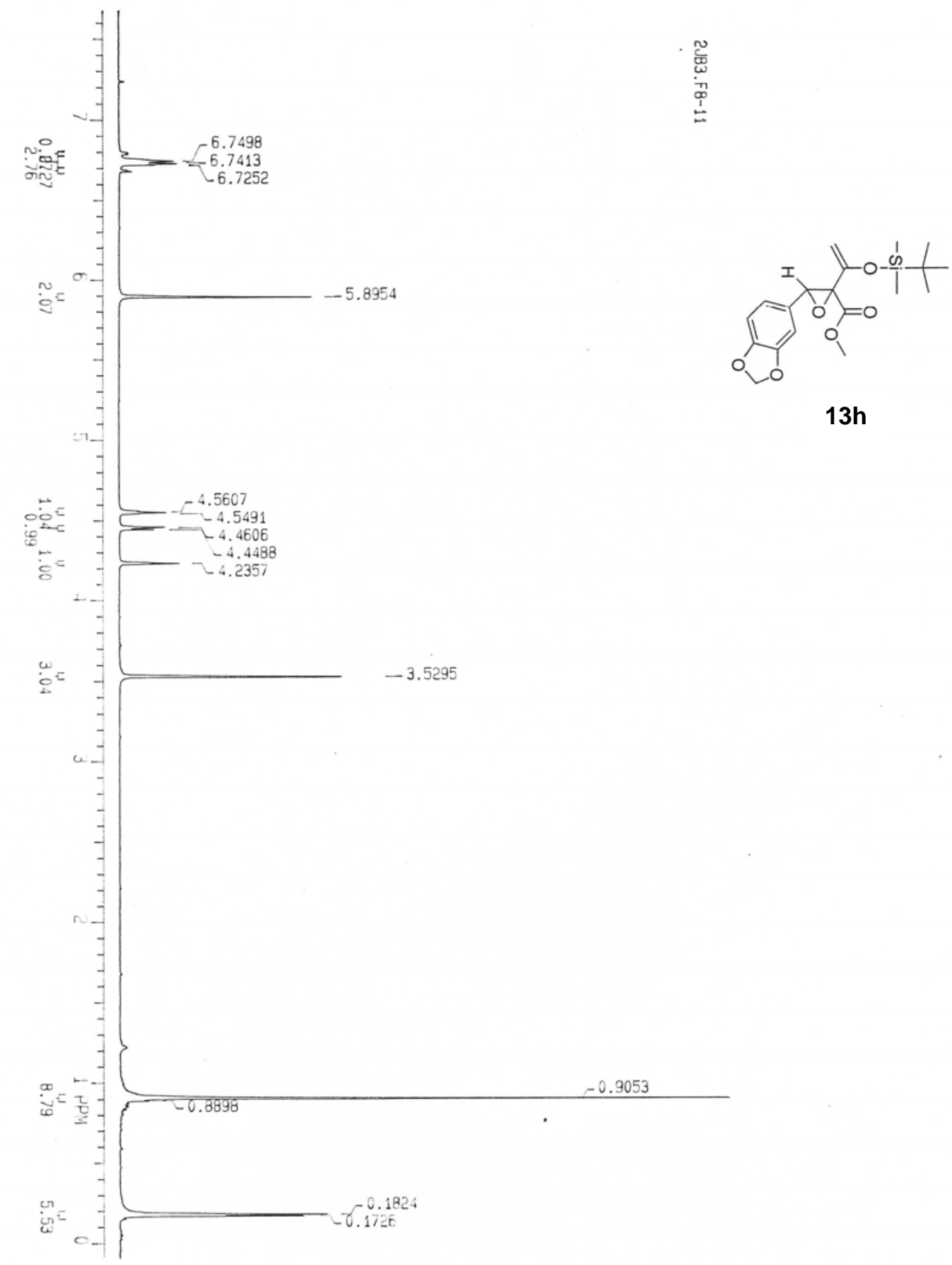




\section{M.P. Doyle et al. - Supporting Information}

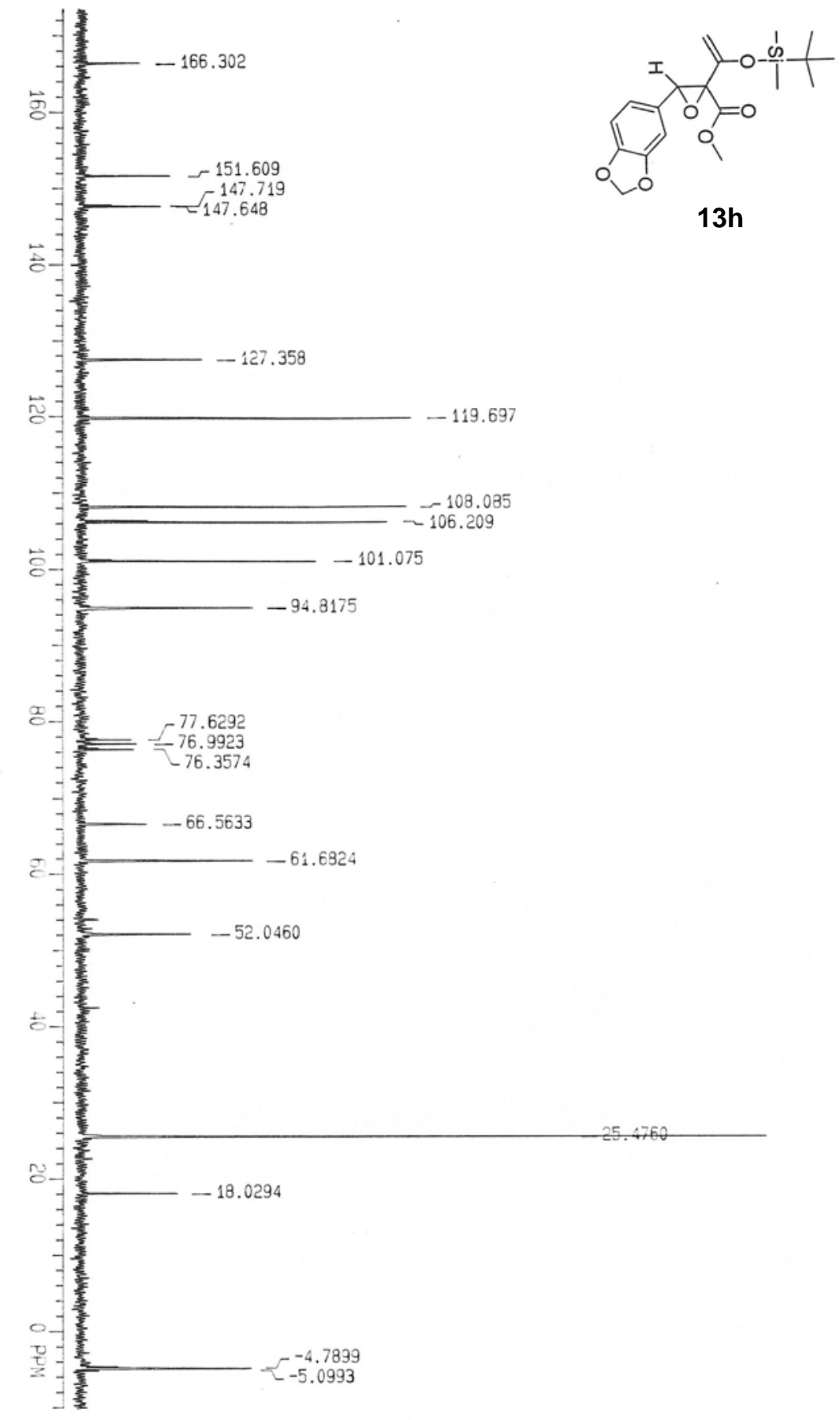




\section{M.P. Doyle et al. - Supporting Information}

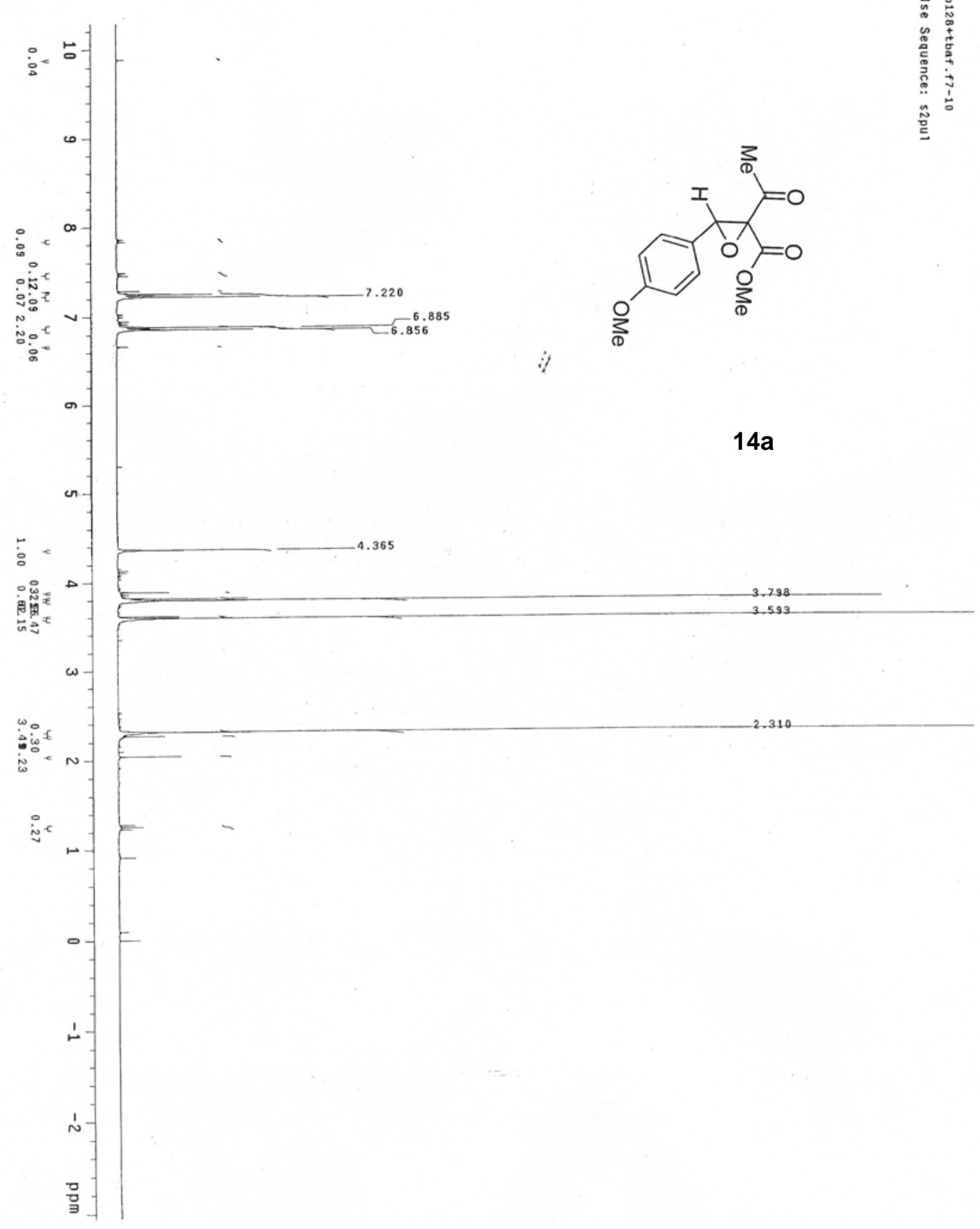




\section{M.P. Doyle et al. - Supporting Information}
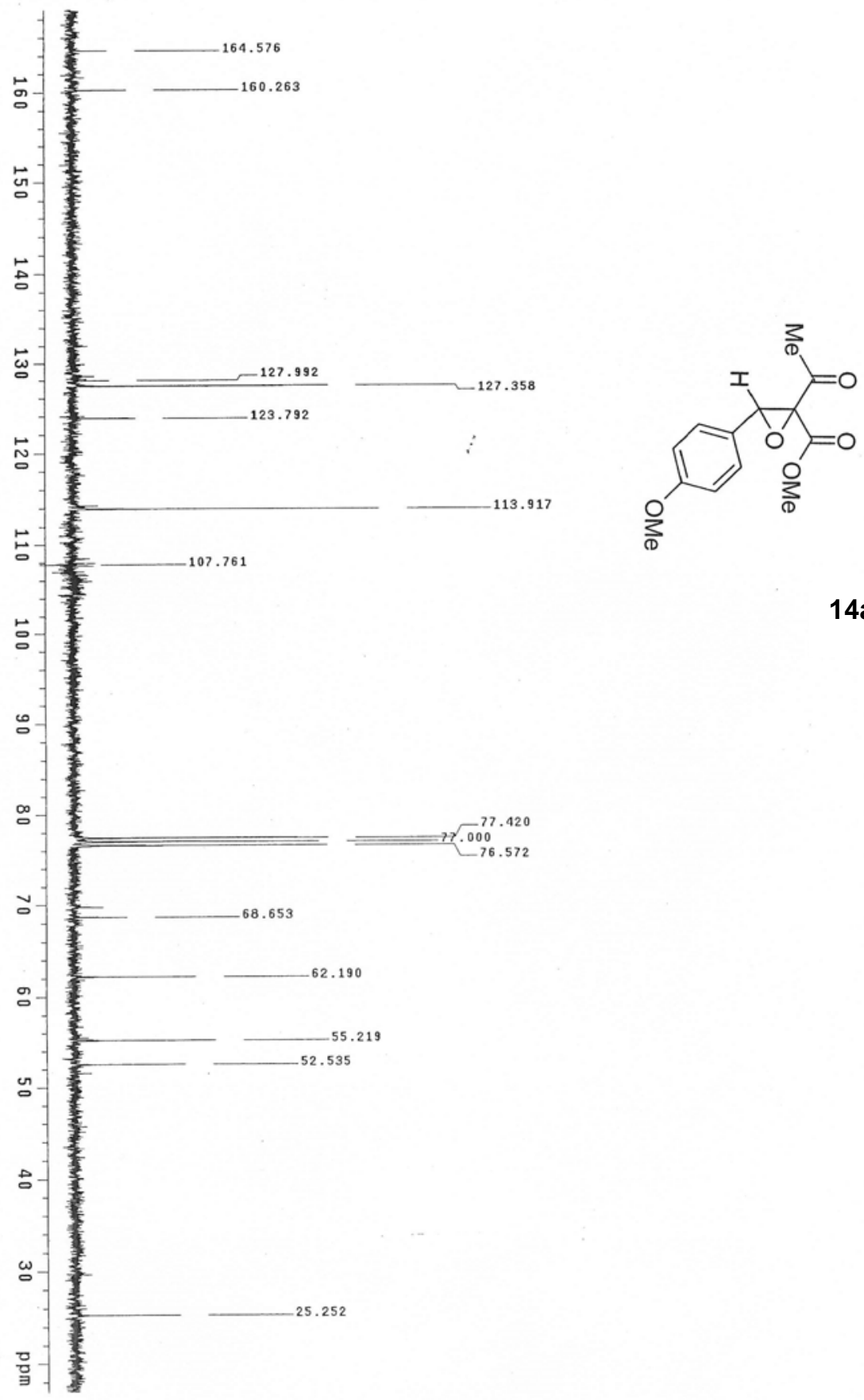

$14 a$

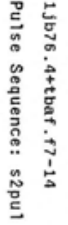




\section{M.P. Doyle et al. - Supporting Information}

Table 1a. Divergence in Product Formation from $\mathrm{Rh}_{2}(\mathrm{OAc})_{4}$ Catalyzed Reactions of Dimethyl Diazomalonate with Excess Aldehyde $^{\mathrm{a}}$

\begin{tabular}{|c|c|c|c|c|}
\hline \multirow[t]{3}{*}{ Entry } & \multirow[t]{3}{*}{$\mathrm{Ar}=$} & isolated yield, $\%^{b}$ & $10: 11^{C}$ & \multirow[t]{3}{*}{ cis-10:trans-10 } \\
\hline & & $10+11$ & 2.0 eq(5.0 eq) & \\
\hline & & \multicolumn{2}{|l|}{$2.0 \mathrm{eq}(5.0 \mathrm{eq})$} & \\
\hline $\mathbf{a}$ & $p-\mathrm{MeOC}_{6} \mathrm{H}_{4}$ & $20(--)$ & $0: 100(--)$ & -- \\
\hline b & $p-\mathrm{MeC}_{6} \mathrm{H}_{4}$ & $17(14)$ & $36: 64(51: 49)$ & $1.5: 1(1.5: 1)$ \\
\hline c & $\mathrm{C}_{6} \mathrm{H}_{5}$ & $35(20)$ & $75: 25(91: 9)$ & $1.4: 1(1.7: 1)$ \\
\hline d & $p-\mathrm{ClC}_{6} \mathrm{H}_{4}$ & $28(4.0)$ & 83:17(95:5) & $1.7: 1(1.7: 1)$ \\
\hline $\mathbf{e}$ & $p-\mathrm{F}_{3} \mathrm{CC}_{6} \mathrm{H}_{4}$ & $39(14)$ & 100:0(100:0) & $1.4: 1(1.4: 1)$ \\
\hline f & $p-\mathrm{NO}_{2} \mathrm{C}_{6} \mathrm{H}_{4}$ & $6.0(--)$ & 100:0(--) & 1.9:1(--) \\
\hline
\end{tabular}

${ }^{a}$ Reactions performed in refluxing dichloromethane with $1.0 \mathrm{~mol} \%$

$\mathrm{Rh}_{2}(\mathrm{OAc})_{4}$ and 2.0 eq or 5.0 eq of aldehyde, based on diazomalonate.

${ }^{b}$ Isolated weight yield of chromatographically pure $\mathbf{1 0}+\mathbf{1 1} .{ }^{c}$ Determined by

${ }^{1} \mathrm{H}$ NMR analyses prior to chromatographic purification. 